\title{
INTEGRATED APPROACH FOR THE PETROPHYSICAL INTERPRETATION OF POST - AND PRE-STACK 3-D SEISMIC DATA, WELL-LOG DATA, CORE DATA, GEOLOGICAL INFORMATION AND RESERVOIR PRODUCTION DATA VIA BAYESIAN STOCHASTIC INVERSION
}

Third Annual Report for the Period

September 30, 2002 to August 30, 2003

By

Carlos Torres-Verdín

Mrinal K. Sen

March 2004

Work Performed under Contract No. DE-FC26-00BC15305

Prepared for U.S. Department of Energy

Purna C. Halder, Project Manager

U.S. Department of Energy

National Energy Technology Laboratory

One West Third Street

Tulsa, Oklahoma 74103

Prepared by

The University of Texas

Center for Petroleum and Geosystems Engineering

$\&$

Institute for Geophysics

Austin, Texas 78712 


\section{DISCLAIMER}

This report was prepared as an account of work sponsored by an agency of the United States Government. Neither the United States Government nor any agency thereof, nor any of their employees, makes any warranty, express or implied, or assumes any legal liability or responsibility for the accuracy, completeness, or usefulness of any information, apparatus, product, or process disclosed, or represents that its use would not infringe privately owned rights. Reference herein to any specific commercial product, process, or service by trade name, trademark, manufacturer, or otherwise does not necessarily constitute or imply its endorsement, recommendation, or favoring by the United States Government or any agency thereof. The views and opinions of the authors expressed herein do not necessarily state or reflect those of the United States Government or any agency thereof. 


\begin{abstract}
The present report summarizes the work carried out between September 30, 2002 and
\end{abstract} August 30, 2003 under DOE research contract No. DE-FC26-00BC15305.

During the third year of work for this project we focused primarily on improving the efficiency of inversion algorithms and on developing algorithms for direct estimation of petrophysical parameters. The full waveform inversion algorithm for elastic property estimation was tested rigorously on a personal computer cluster. For sixteen nodes on the cluster the parallel algorithm was found to be scalable with a near linear speedup. This enabled us to invert a 2D seismic line in less than five hours of CPU time. We were invited to write a paper on our results that was subsequently accepted for publication. We also carried out a rigorous study to examine the sensitivity and resolution of seismic data to petrophysical parameters. In other words, we developed a full waveform inversion algorithm that estimates petrophysical parameters such as porosity and saturation from pre-stack seismic waveform data. First we used a modified Biot-Gassmann equation to relate petrophysical parameters to elastic parameters. The transformation was validated with a suite of well logs acquired in the deepwater Gulf of Mexico. As a part of this study, we carried out a sensitivity analysis and found that the porosity is very well resolved while the fluid saturation remains insensitive to seismic wave amplitudes. Finally we conducted a joint inversion of pre-stack seismic waveform and production history data. To overcome the computational difficulties we used a simpler waveform modeling algorithm together with an efficient subspace approach. The algorithm was tested on a realistic synthetic data set. We observed that the use of pre-stack seismic data helps tremendously to improve horizontal resolution of porosity maps. Finally, we submitted four publications to refereed technical journals, two 
refereed extended abstracts to technical conferences, and delivered two oral presentation at a technical forum. All of these publications and presentations stemmed from work directly related to the goals of our DOE project. 


\section{TABLE OF CONTENTS}

DISCLAIMER

ABSTRACT. III

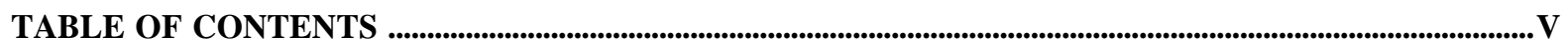

TABLE OF FIGURES ........................................................................................................................................

TABLE OF TABLES .........................................................................................................................................................

1. INTRODUCTION .........................................................................................................................................................................................

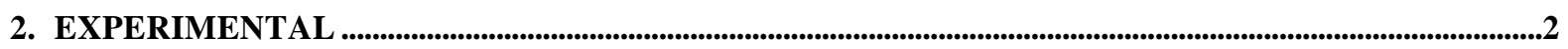

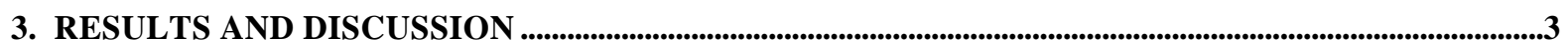

3.A. CENTER FOR PETROLEUM AND GEOSYSTEMS ENGINEERING......................................................................

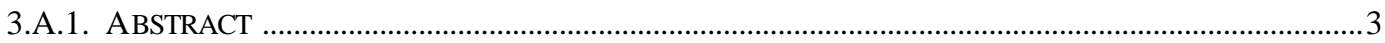

3.A.2. JOINT INVERSION OF PRESTACK 3D SEISMIC DATA AND TIME RECORDS OF FLUID PRODUCTION

MEASUREMENTS: A NUMERICAL STUDY......................................................................................

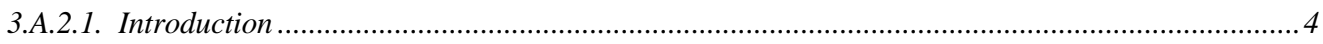

3.A.2.2. Production History Matching and Seismic Data Inversion ........................................................

3.A.2.3. On the Choice of a Model Covariance Matrix ……………………………………................. 13

3.A.2.4. Rock Physics/Fluid Substitution Model .................................................................................. 14

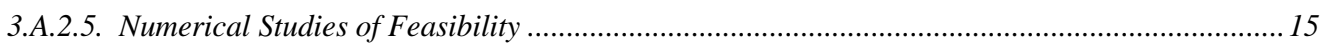

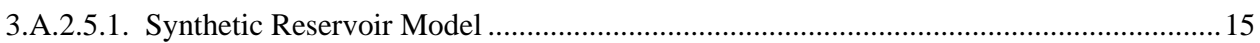

3.A.2.5.2. Inversion of Time Records of Fluid Production Data.......................................................2

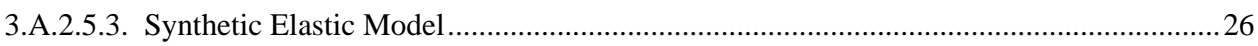

3.A.2.5.4. Inversion of Pre-stack Seismic Data........................................................................ 28

3.A.2.5.5. Assessment of the Data Subspace Method ......................................................................... 38

3.A.2.5.6. Joint Inversion of Pre-stack Seismic Data and Time Records of Fluid Production

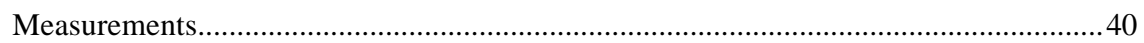

3.A.2.6. Discussion and Conclusions.............................................................................................4 42

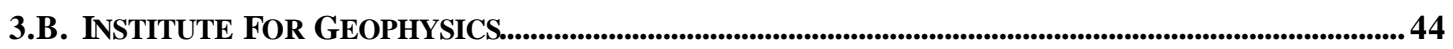

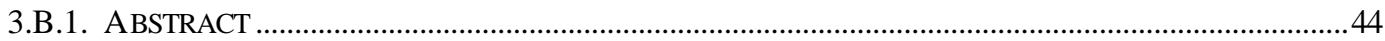

3.B.2. FULL W AVEFORM SEISMIC INVERSION USING A DISTRIBUTED SYSTEM OF COMPUTERS........44

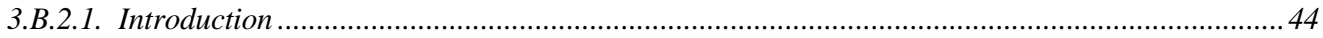

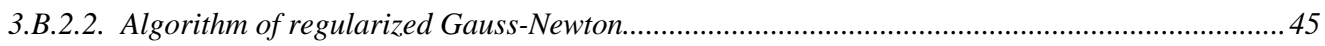

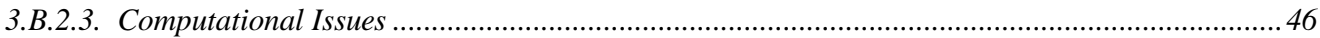

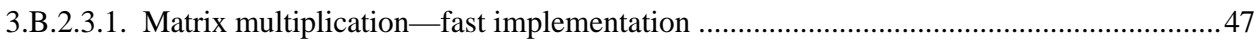

3.B.2.3.2. Truncated Regularized Gauss-Newton ......................................................................... 48

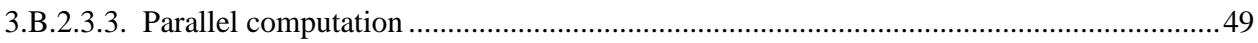

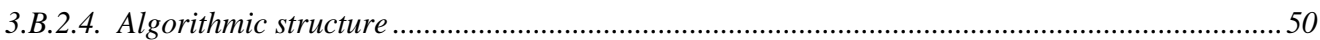

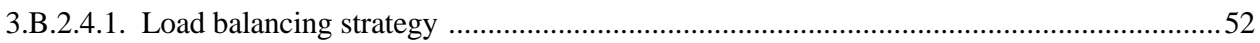

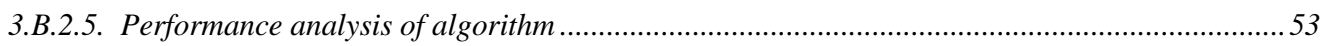

3.B.2.5.1. Elapsed time vs. number of processors.....................................................................55 
3.B.2.5.2. Speedup, overhead, efficiency, performance measure and efficacy on homogeneous clusters.

3.B.3. DIRECT ESTIMATION OF PETROPHYSICAL PARAMETERS VIA FULL WAVEFORM INVERSION OF

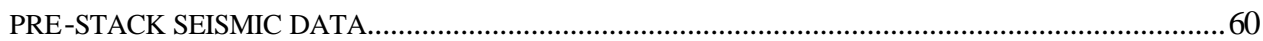

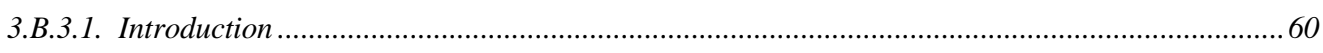

3.B.3.2. Relation between rock physics and petrophysical properties ..................................................61

3.B.3.3. Sensitivity of $P$ - and $S$-wave velocities to porosity and saturation ............................................66 66

3.B.3.4. Determination of petrophysical parameters and seismic velocities from well logs................... 70

3.B.3.5. Waveform inversion in the determination of petrophysical parameters................................... 73

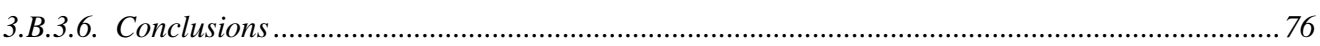

3.B.3.7. Appendix 176

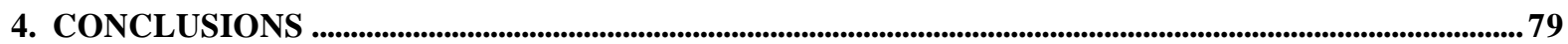

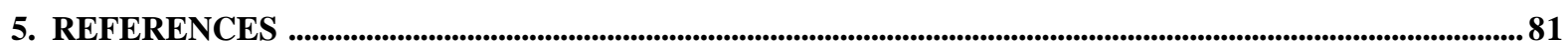

6. APPENDICES

6.A. MANUSCRIPTS SUBMITTED FOR PUBLICATION IN REFEREED TECHNICAL JOURNALS ................ 84

6.B. EXPANDED REFERENCED CONFERENCE ABSTRACTS .....................................................................8 


\section{TABLE OF FIGURES}

Figure 3.A.1. Graphical description of the synthetic 3D reservoir model. Well locations are indicated with vertical lines penetrating through the reservoir. The injection well is located close to the center of the reservoir. There are four production wells symmetrically located around the injection well.

Figure 3.A.2. Plan view and cross section of the reservoir model shown in figure 3.A.1. The plan view indicates locations of the injector and producer wells together with the geometrical dimensions of the reservoir. A regular Cartesian grid inserted within the reservoir indicates the location, dimensions, and number of discretization blocks used to describe the spatial distribution of reservoir properties.

Vertical dimensions of the reservoir and of the discretization blocks are shown om the cross-section.. 18

Figure 3.A.3. Water-oil relative permeability curves used in the numerical simulation of the water-oil system. 19

Figure 3.A.4. Capillary pressure curve used in the numerical simulation of the water-oil system.

Figure 3.A.5. Graph depiction of the true spatial distribution of porosity.

Figure 3.A.6. Graph depiction of the true spatial distribution of log-permeability [log (md)].

Figure 3.A.7. Graphical description of the spatial distribution of log-permeability estimated from the inversion of time records of water-oil ratio. [ $\log (\mathrm{md})]$.

Figure 3.A.8. Graphical description of the spatial distribution of porosity estimated from the inversion of time records of water-oil ratio.

Figure 3.A.9. Comparison of the measured and numerically simulated time record of water-oil ratio (wor) for the production well no. 1 . The measured data were contaminated with $5 \%$ additive gaussian random noise.

Figure 3.A.10. Comparison of the measured and numerically simulated time record of water-oil ratio (wor) for the production well no. 2 . The measured data were contaminated with $5 \%$ additive gaussian random noise.

Figure 3.A.11. Comparison of the measured and numerically simulated time record of water-oil ratio (wor) for the production well no. 3 . The measured data were contaminated with $5 \%$ additive gaussian random noise.

Figure 3.A.12. Comparison of the measured and numerically simulated time record of water-oil ratio (wor) for the production well no. 4 . The measured data were contaminated with $5 \%$ additive gaussian random noise.

Figure 3.A.13. Graphic representation of the ricker wavelet used in the simulation of pre -stack seismic data...27

Figure 3.A.14. Graphic depiction of the spatial distribution of porosity inverted from pre-stack seismic data...29

Figure 3.A.15. Graphic depiction of the spatial distribution of porosity used to initialize the inversion of prestack seismic data.

Figure 3.A.16. Graphic depiction of the statistical distribution of porosity values in the synthetic reservoir model.

Figure 3.A.17. Graphic depiction of the statistical distribution of porosity values in the reservoir model inverted from pre-stack seismic data 
Figure 3.A.18. Comparison of the measured and simulated seismic traces for a single cmp gather: panel (a) shows the measured seismic data and panel, (b) shows the numerical simulated seismic data. The measured data were contaminated with $5 \%$ additive gaussian random noise.

Figure 3.A.19. Plan view of the statistical correlation between the observed pre-stack seismic data and the prestack seismic data simulated from the uniform reservoir model used to initialize the inversion..............33

Figure 3.A.20. Plan view of the statistical correlation between the observed pre-stack seismic data and the prestack seismic data simulated from the inverted reservoir mode...

Figure 3.A.21. Vertical profiles of p-wave velocity: (a) initial velocity, (b) true model, and (c) estimated model from waveform inversion.

Figure 3.A.22. Vertical profiles of s-wave velocity: (a) initial velocity, (b) true model, and (c) estimated model from waveform inversion

Figure 3.A.23. Graphic depiction of the spatial distribution of porosity inverted from pre-stack seismic data assuming a low frequency ricker wavelet (central frequency $=20 \mathrm{hz}$ ).

Figure 3.A.24. Graphic depiction of the spatial distribution of porosity for the case of a tight synthetic reservoir model.

Figure 3.A.25. Graphic depiction of the spatial distribution of porosity inverted from pre-stack seismic data simulated for the case of the tight reservoir model shown in figure 3.A.23.

Figure 3.A.26. Simulated pre-stack $\mathrm{cmp}$ gather for the case of the tight reservoir model shown in figure $23 \ldots . . .38$

Figure 3.A.27. Evolution of the cost function used for inversion as a function of iteration number. The cost function is constructed using the data subspace algorithm described by equation (3.A.6). Case a and case $\mathrm{b}$ correspond to different porosity models used to initialize the inversion

Figure 3.A.28. Graphical description of the spatial distribution of porosity inverted from pre-stack seismic data simulated for the tight reservoir model shown in figure 3.A.23.

Figure 3.A.29. Graphic depiction of the spatial distribution of log-permeability $[\log (\mathrm{md})]$ obtained from the joint inversion of pre-stack seismic data and time records of water-oil ratio. Data input to the inversion were generated from the tight reservoir model shown in figure 3.A.23.

Figure 3.B.1. Plots of differential seismograms with respect to p-wave velocity (top row), s-wave velocity (middle row) and density (bottom row) of different layers of an earth model for three different values of ray-parameters $(0.0,0.1$ and $0.2 \mathrm{sec} / \mathrm{km}$ at the left, moiddle and right column respectively): each differential seismogram is a vector of frechet derivatives with respect to model parameters. It is obvious that for $\mathrm{p}=0$, the seismograms are not sensitive to changes in the shear wave velocity.

Figure 3.B.2. Schematic plot of algorithmic structure. The data are distributed from master node to several nodes (slave). Note that algorithm uses single program multiple data (xpmd) type parallel computation. $\mathrm{E}_{i}, \mathrm{~s}_{i}, \mathrm{~g}_{i}$ correspond to the partial values of error function, sensitivity matrix and $\mathrm{g}^{t} \mathrm{~g}$ computed at processor $i$. The totals $\mathrm{e}_{i}, \mathrm{~s}_{i}, \mathrm{~g}_{i}$ are computed using a global 2 sum.

Figure 3.B.3. (a) plot of synthetically generated $t-p$ seismograms with $5 \%$ random noise derived from a true earth model presented in the adjacent panel. The source wavelet used is ricker wavelet with $35 \mathrm{hz}$ peak frequency. (b) plot of p-, s-velocities and density with two-way time. Note that the layer thickness is expressed in terms of two -way travel time by $\mathrm{p}$-wave in a medium. 
Figure 3.B.4. (a) the plot of the elapsed time versus the number of processors. Elapsed time decreases with the addition of the processor.

Figure 3.B.4. (b) plot of the elapsed time versus the inverse of the number of processors: the plot is almost linear, indicating that the sequential components of the algorithm are nearly constant. Similar trends are observed for the two datasets containing 30 and 60 traces

Figure 3.B.5. (a) plot of speedup versus the number of processors: speedup curve nearly follows a linear trend indicating good scalability. (b) plot of overhead versus the number of processors, and (c) plot of efficiency versus the number of processors. Similar trends are observed for the two datasets................58

Figure 3.B.6. (a) plot of data fir presented in three panels; the left panel is observed data, the middle panel id the best fit data and the right panel is the data residual. (b) plots of true (solid line), initial guess (dashed line), and the inverted model (dotted line)......

Figure 3.B.7. Plot of sensitivities for both p- (solid line) and s-wave (broken line) velocities with respect to porosity versus porosity for different values of saturation and clay content. Curves presented in the upper panel are for clean sand saturated with water or water and gas while curves in the lower panel are fordirty sand contaminated with $15 \%$ clay content sensitivities for both $\mathrm{p}$ - and s-wave vary strongly with porosity for any value of saturation.

Figure 3.B.8. Plot of sensitivities for both p- (solid line) and s-wave (broken line) velocities with respect to saturation versus saturation for different value of porosity and clay content. Curves presented in the upper panel are for clean sand saturated with water or water and gas while curves in lower panel are for dirty sand contaminated with $15 \%$ clay. Both $\mathrm{p}$ - and s- velocity remain flat for most of the saturation value except near to the higher end of saturation, where it shows a vary strong variation.

Figure 3.B.9. (a) plot of p-velocities and (b) plot of s-velocities with respect to porosity for different values of saturation. The continuous line corresponds to $100 \%$ water saturation, the line with open circle corresponds to $90 \%$ saturation, and the line with filled circles corresponds to $60 \%$ saturation. Model considered is clean sand saturated either with water or water and gas. Panel (c) is the vp/vs ratio versus porosity, which continues to increase nonlinearly with porosity and the nature of curves remains the same irrespective of saturation.

Figure 3.B.10. (a) contour maps of p- velocity and (b) contour maps of s-velocity as functions of porosity and saturation.

Figure 3.B.11. Plots of wireline-log-derived porosity (blue line), water saturation (orange line) and percent clay volume (green line) with depth..

Figure 3.B.12. Plots of log-measured (magenta line) and predicted (blue line) values of $\mathrm{p}-\mathrm{s}$-velocities and bulk density with depth.

Figure 3.B.13. Plot of predicted values of $\mathrm{p}-\mathrm{s}$-velocities and bulk density with two-way vertical travel time in (ms).

Figure 3.B.14. Synthetically generated $\tau-p$ seismic section using the time converted velocities and the density profile shown in figure 3.B.7.

Figure 3.B.15. Plot of inverted porosity profile (magenta line) overlaid on true profile (blue line).

Figure 3.B.16. Plot of $\tau-p$ seismic section after inversion (left panel) and the original one (right panel)... 


\section{TABLE OF TABLES}

Table 3.A.1. Summary of fluid and reservoir properties used to construct the synthetic reservoir model and to numerically simulate time records of fluid production data ..................................................................................2

Table 3.A.2. Descriptions of the bulk modulus, shear modulus, and bulk density of the rock and fluid constituents assumed in the construction of the synthetic reservoir model.

Table 3.A.3. Summary of the characteristics of the pre-stack seismic data used in this paper to generate synthetic waveforms 


\section{INTRODUCTION}

This annual report describes work performed by the Institute for Geophysics and the Center for Petroleum and Geosystems Engineering, both with The University of Texas at Austin, between September 30, 2002 and August 30, 2003 under DOE research contract No. DE-FC26-00BC15305.

Work performed by the Center of Petroleum and Geosystems Engineering includes joint inversion of pre-stack seismic data and fluid flow measurements.

During the same funding period, the UTIG group conducted two main tasks:

(a) Rigorous Evaluation of Parallel pre-stack waveform inversion code in the $\tau$-p domain,

(b) Theoretical development and numerical implementation of a pre-stack inversion code for direct estimation of petrophysical parameters.

The section Results and Conclusions of this report provides a detailed technical summary of the above developments. 


\section{EXPERIMENTAL}

There are no experimental components of the project to be included in this annual report. The tasks and components of the project comprise the development of numerical algorithms and computer software. Likewise, the project includes analysis and interpretation of both field and numerically simulated data. All of these developments are summarized in the section Results and Conclusions of the report. 


\section{RESULTS AND DISCUSSION}

\section{A. Center for Petroleum and Geosystems Engineering}

\section{A.1. ABSTRACT}

Work performed by the Center for Petroleum and Geosystems Engineering comprised the joint inversion of reservoir production measurements and 3D pre-stack seismic data. We developed an inversion approach for estimating hydrocarbon reservoir properties that jointly honor multi-offset seismic data and time records of fluid production measurements. We consider the example of a synthetic reservoir model consisting of spatially heterogeneous sands saturated with oil and subject to water flooding. Spatial discretization of the reservoir is performed with a set of non-overlapping and uniform rectangular blocks. The objective is to estimate spatial distributions of porosity and permeability under the assumption that the initial water saturation is at capillary equilibrium. A Biot-Gassmann rock physics/fluid substitution model is used to quantitatively link P-wave velocity, S-wave velocity, and bulk density to total porosity and fluid saturation. Numerical experiments show that the enforcement of a physics/fluid substitution model effectively constrains the solution space of the inversion thereby reducing non-uniqueness and hence providing a more accurate and stable estimation of the spatial distribution of porosity.

Examples of inversion with noisy synthetic measurements indicate that the use of prestack seismic data in conjunction with time records of fluid production can significantly improve the spatial resolution of reservoir parameters. In particular, a good reconstruction of the spatial distribution of porosity is obtained when the mean value of porosity is above $10 \%$. The synthetic examples also show that the joint inversion of pre-stack seismic data and water-oil ratio measurements does not provide a good estimation of the spatial distribution of 
permeability. Accurate and reliable estimation of permeability requires of a strong statistical correlation between permeability and porosity, or else of the use of additional independent data such as permanent in-situ pressure measurements or time records of bottom-hole pressure.

The joint inversion of pre-stack seismic data and production measurements requires a significant amount of computations that cannot be approached with a naïve implementation of least-squares nonlinear optimization. To circumvent this difficulty, we introduce a novel data subspace inversion approach that effectively compresses the data space and therefore substantially reduces computer memory and number of operations required to calculate the data sensitivity matrix. The same approach can be used to perform the inversion of different combinations of data subspace vectors.

\section{A.2. JOINT INVERSION OF PRESTACK 3D SEISMIC DATA AND TIME RECORDS OF FLUID PRODUCTION MEASUREMENTS: A NUMERICAL STUDY}

\section{A.2.1. Introduction}

Prediction of dynamic reservoir behavior depends on the accurate description of the spatial distribution of reservoir parameters. Matching production data at each well is one of the most commonly used strategies for estimating spatial distributions of reservoir parameters. Simulation of multi-phase fluid-flow behavior is inherently a highly nonlinear problem. The corresponding production history matching is not only mathematically and computationally challenging, but also highly unstable and non- unique. In particular, the nonuniqueness of production history matching is prominent because production measurements are only available at limited well locations. It is also common practice to acquire production measurements along a depth interval that includes several flow units in the same well. 
Because of this, production measurements represent long vertical averages of reservoir properties that exhibit relatively low sensitivity to lateral variations of porosity, permeability, and fluid saturation. Standard reservoir history matching procedures are based on geostatistical methods that populate petrophysical parameters in the inter-well region. In so doing, a spatial covariance matrix is estimated from available rock-core and well-log data to enforce a degree of spatial smoothness for the geostatistically interpolated reservoir parameters. The inversion consists of adjusting the free parameters of the spatial covariance matrix in order to numerically reproduce the measured time records of fluid production measurements (Chu et al., 1996; Wu et al., 1999). Reservoir history matching practitioners usually resort to geostatistical procedures as a palliative for non-uniqueness and instability.

Three-dimensional seismic data and seismic attributes have been used to guide the geostatistical interpolation of inter-well properties through co-kriging and stochastic simulation techniques. More recently, geostatistical approaches have been used to invert reservoir petrophysical properties that simultaneously honor amplitude variations of poststack seismic data and well logs. For example, Hass and Dubrule (1994), Behrens and Tran (1998), and Grijalba-Cuenca and Torres-Verdin (2000) applied geostatistical simulation and inversion to generate a set of reservoir models that honored the post-stack seismic data and the existing well logs. The seismic data are laterally dense and hence can be used to fill the spatial gap between sparse well locations. At the same time, several researchers have advocated the use of pre-stack seismic waveform inversion to estimate petrophysical properties (Tarantola et al., 1984; Kolb et al., 1986; Mora 1987; Sen and Stoffa, 1991). Pre-stack seismic data exhibit more degrees of freedom to estimate elastic and petrophysical parameters than post-stack seismic data. For instance, pre-stack seismic data can provide 
estimates of P-wave velocity, S-wave velocity, and bulk density, whereas post-stack seismic data can only provide estimates of P-wave acoustic impedance (the product of bulk density times P-wave velocity). However, in similarity with production history matching, the inversion of pre-stack seismic waveform data suffers from non-uniqueness and high computational cost. Global minimization methods such as simulated annealing and genetic algorithms, which could be efficient for pre-stack seismic inversion, are not suitable for reservoir history matching because one cannot afford the numerous iterations entailed by such algorithms. It would be necessary to resort to an impractical amount of computer time to repeatedly solve the multi-phase flow equations.

In comparison to traditional reservoir simulation and seismic modeling techniques, little has been reported in the open technical literature on the quantitative use of pre-stack seismic data to constrain the process of reservoir history matching. In this paper, we develop a data subspace approach for the joint inversion of pre-stack seismic waveform data and time records of fluid production measurements. The proposed method differs from existing inversion approaches in several aspects. First, unlike traditional inversion methods inverting P-wave and S-wave velocities, the proposed method focuses on estimating the spatial distribution of porosity, which is related to $\mathrm{P}$ - and S-wave velocities through a simple BiotGassmann rock physics/fluid substitution model. The physics/fluid substitution model can effectively constrain the solution space of the inversion thereby reducing non- uniqueness and hence rendering more accurate estimates of the spatial distribution of porosity. Second, a new data subspace algorithm is developed to constructively combine pre-stack seismic waveform and production data in the estimation of realistic reservoir models. The specific basis used to represent the data subspace vectors is chosen according to the type of available 
measurements rather than according to the types of inversion parameter. The outstanding advantage of the new data subspace inversion method is its low computational cost and computer memory requirements due to the reduction of both the number of sensitivity calculations and the size of the system of normal equations. Thus, large-scale inversions can be approached in an efficient manner. Third, an adjoint technique is adopted to generate the sensitivity matrix of production data with respect to reservoir parameters.

We consider three types of 3D synthetic reservoir models to illustrate and assess the efficiency of our proposed inversion approach. Synthetic reservoir models consist of spatially heterogeneous sands saturated with oil and irreducible water saturation. The reservoir is subject to water injection with one injector and four producer wells. We make the assumption that the initial water saturation is in capillary equilibrium and pose the inversion as the estimation of the spatial distributions of total porosity and permeability without imposing a statistical correlation between the two petrophysical parameters.

\section{A.2.2. Production History Matching and Seismic Data Inversion}

Production history matching can be stated as an optimization problem: minimizing the misfit between the observed data, $d_{o b s}$, and the data simulated numerically for a given model, $m$. Based on a simple Bayesian statistical rule, the model parameters can be inverted by minimizing the following objective function

$$
J(\mathbf{m})=\left(\mathbf{m}-\mathbf{m}_{o}\right)^{T} C_{M}^{-1}\left(\mathbf{m}-\mathbf{m}_{0}\right)+\left(g-\mathbf{d}_{o b s}\right)^{T} C_{D}^{-1}\left(g-\mathbf{d}_{o b s}\right),
$$

where $\underline{\mathbf{m}}$ is the vector of reservoir parameters, $C_{D}$ is the data covariance matrix, $C_{M}$ is the model covariance matrix, and $\mathbf{m}_{o}$ is an a-priori estimate of the reservoir parameters. In equation (1), the model vector, $\mathbf{m}$, is a finite-size vector that contains all of the unknowns 
that completely describe the spatial distribution of reservoir parameters. A convenient way to construct the model vector is $\mathbf{m}=(\log k, \phi)^{T}$ where $k$ is absolute permeability and $\phi$ is total porosity. We choose a logarithmic transformation for permeability because of two reasons: one is to naturally compress the wide variability of this parameter, and the second is to naturally enforce positivity for the estimated value. The subscript $o b s$ in equation (3.A.1) is used to identify the available measurements, i.e., time records of fluid production measurements, one measurement per sampling time measured after the onset of production.

When the number of unknown reservoir parameters is large and the number of independent degrees of freedom in the measurements is small, numerous combinations of parameters can equally satisfy the time records of fluid production measurements. Therefore, the first additive term in equation (3.A.1) is intended to reduce the non-uniqueness of the solution as well as stabilize the estimation of $\mathbf{m}$ in the presence of noisy data. Precisely speaking, the first term leads the inversion toward a specific set of solutions in parameter space. Inversion of pre-stack seismic data alone can be approached with the use of an objective function similar to that described by equation (3.A.1), except that the vector of unknown parameters would contain values of P-wave velocity, S-wave velocity, and bulk density. In addition, the observed data would be described with a data vector $\mathbf{d}_{o b s}$ that would contain time-domain amplitudes of normalized vertical displacement for a particular sourcereceiver distance.

Generally, the Gauss-Newton mnlinear minimization algorithm is efficient for locating the local minima of a differentiable objective function. The standard Gauss-Newton method requires repeatedly solving a normal system of equations for which the number of linear equations is larger or equal to the number of unknown parameters. For the problem at 
hand, we have far more unknown parameters than independent measurements. Solving the system of linear equations becomes computational prohibitive. In both geophysics and reservoir engineering areas, a modified Gauss-Newton approach is adopted for the minimization of equation (3.A.1), and this leads to the fixed-point iterated solution

$$
\mathbf{m}_{l+1}=\mathbf{m}_{o}-C_{M} G_{l}^{T}\left[C_{D}+G_{l} C_{M} G_{l}^{T}\right]^{-1}\left[g-\mathbf{d}_{o b s}+G_{l}\left(\mathbf{m}_{l}-\mathbf{m}_{o}\right)\right],
$$

where $G_{l}$ is the matrix of sensitivity coefficients calculated at the $l$-th iteration. Because of the high computational cost required to calculate the sensitivity matrix of waveform data with respect to the unknown parameters, it would be impractical if seismic data inversion were conducted in a global 3D manner. In this study, we adopt a local $1 \mathrm{D}$ seismic data inversion technique to reproduce the seismic amplitude measurements. This is equivalent to assuming that the measured pre-stack seismic data is the response of a locally 1D medium consisting of horizontal layers. Each pre-stack, or CMP seismic gather, is treated independently to approach the inversion of a 1D medium. The "mosaique" of 1D pre-stack seismic inversion is taken as the $3 \mathrm{D}$ spatial distribution of elastic parameters. For the production data, we apply the adjoint technique to compute the sensitivity of fluid production data to a perturbation of the vector of model parameters, m (Wu et. al, 1999). At every iteration, we solve the linear system of equations given by

$$
\left[C_{D}+G_{l} C_{M} G_{l}^{T}\right] \underline{\mathbf{x}}=g-\mathbf{d}_{o b s}+G_{l}\left(\mathbf{m}_{l}-\mathbf{m}_{o}\right)
$$

for the model update vector, $\underline{\boldsymbol{x}}$. In this formulation, the number of equations is equal to the number of measurements. Such a modified Gauss-Newton method operates efficiently for cases where the size of the vector $\mathbf{d}_{o b s}$ is small. Specifically, the adjoint technique for computing sensitivities only solves an adjoint system with multiple right-hand sides, equal to the number of production data. Since the number of production data is much less than the 
number of unknown parameters, both computational cost and memory become affordable for generating and storing the sensitivity coefficient matrix of production data with respect to reservoir parameters. However, for the inversion of pre-stack seismic data, if the sensitivity coefficients of each sample point of multioffset gathers with respect to reservoir parameters were computed, the size of the sensitivity matrix would increase dramatically. The high computational cost incurred by the large-size sensitivity matrix involved in the GaussNewton iteration renders the minimization approach impractical. Thus, the development of more efficient inversion algorithms becomes crucial when pre-stack seismic data and production data are combined for inversion. In this paper, we make use of a new data subspace approach to efficiently assimilate the large amount of data input to the joint inversion of pre-stack seismic data and time records of fluid production measurements. Our data subspace approach is different from Kennett et al's (1988), Oldenburg et al.'s (1993) and Abacuioglu et al.'s (2001) in that we partition the data objective function based on the types of observed data rather than on the types of model parameters. Therefore, not only can the dimensions of the sensitivity matrix be significantly reduced but also the direct computation of the sensitivity of individual data points with respect to model parameters can be avoided entirely.

Suppose that numerical modeling errors can be neglected and that only measurement errors are of significance. The model response and the measured data can be written as

$$
g_{i, j}=d_{i, j}+e_{i, j} \text { for } i=1, \cdots N_{W} \text { and } j=1, \cdots N_{D} \text {, }
$$

where $\underline{e}$ denotes the vector of measurement errors, $N_{w}$ is either the number of traces or the number of producer wells, $N_{D}$ is either the number of time samples for each seismic trace or the number of time samples of production data for each well. Kennett's reflectivity method 
was used to generate the synthetic pre-stack waveforms in the space and time domains assuming no multiple reflections and no transmission losses. As previously emphasized, under the assumption of a diagonal data covariance matrix, the inversion can be approached by minimizing the objective function defined by equation (3.A.1). Under the assumption of a diagonal data covariance matrix, the second additive term in equation (3.A.1) can be rewritten as

$$
\sum_{i=1}^{N_{W}} \sum_{j=1}^{N_{D}} \frac{\left(\mathrm{g}_{\mathrm{i}, \mathrm{j}}-\mathrm{d}_{\mathrm{i}, \mathrm{j}}\right)^{2}}{\sigma_{\mathrm{i}, \mathrm{j}}^{2}} .
$$

The motivation for our new data subspace inversion approach comes from the central limit theorem. This theorem states that the distribution of the sum of a large number of identically distributed random variables will be approximately normal, regardless of the characteristics of the individual distributions. If the error between the observed data and the model predicted data falls within the range of one standard deviation, it then follows that

$$
u_{i}=\frac{1}{N_{d}} \frac{1}{N_{w}} \sum_{i=1}^{N_{w}} \sum_{j=1}^{N_{d}} \frac{e_{i, j}^{2}}{\sigma_{i, j}^{2}} \rightarrow 1 .
$$

Based on this last property, the joint inversion problem can be stated as follows: Adjust the model parameters by making the limit defined by equation (3.A.6) approach one as closely as possible. If the observed data are matched with the model predicted data, then the functional $u_{i}$ should approach one. Therefore, the objective function defined by equation (3.A.1) can be rewritten as

$$
J_{1}(\mathbf{m})=(\mathbf{u}-\mathbf{e})^{T} C_{M}^{-1}(\mathbf{u}-\mathbf{e})+\left(\mathbf{m}-\mathbf{m}_{0}\right)^{T} C_{M}^{-1}\left(\mathbf{m}-\mathbf{m}_{0}\right)
$$


where $C_{B}$ denotes a weighting matrix that assigns relative importance to the various trace offsets, and to the various production wells, and e is a unity vector given by $\mathbf{e}=(1, \Lambda, 1)^{T}$. It must be emphasized that only the sensitivities of the sub-objective function with respect to model parameters $\frac{\partial u_{i}}{\partial m}$ are required for the minimization of the quadratic cost function defined by equation (3.A.7). Because of this, the proposed data subspace method becomes superior to existing inversion methods as it entails much lower computational costs and computer memory.

The gradient of sub-objective function could be computed using finite differences. However, such an approach is not efficient because finite differences would require the computation of the sensitivities for each data point with respect to each unknown reservoir parameters. In this paper, we extend the adjoint approach (Wu et al., 1999) to compute the gradient of the sub-objective function with respect to model parameters without explicitly calculating the sensitivities of the data with respect to the model parameters. In the limit, if each observed sample point and each production data were chosen as a subspace, the data subspace approach would be equivalent to a standard Gauss-Newton minimization method. On the other hand, the proposed data subspace minimization method would become equivalent to the steepest descent algorithm if a single subspace vector were used for the inversion. A trade-off between the convergence rate and the number of subspace vectors must be made in practice. In particular, the choice of too few subspace vectors results in slow convergence. Conversely, the use of too many subspace vectors suffers from the high cost of computing a large number of gradients of subspace vectors with respect to model parameters. A practical alternative is to construct data subspace vectors that bear similar information. For instance, the data subspace vectors could be constructed with water-oil 
ratio data from a production well, amplitudes from a trace, or measurements taken from a particular time interval, etc. In the following sections, the proposed data subspace inversion method is applied to the independent and joint inversion of pre-stack seismic data and time records of fluid production measurements.

\section{A.2.3. On the Choice of a Model Covariance Matrix}

Under a Bayesian framework, a priori information about the spatial distribution of the unknown reservoir parameters can be enforced with the use of the model covariance matrix (Tarantola, 1987). However, in most cases, the model covariance matrix may not be available due to the lack of a-priori knowledge about the model. Often, the Laplacian or some other smoothing isotropic operator can be applied to regularize the unknown vector of model parameter (Tikhonov and Arsenin, 1977). In particular, Tarantola (1987) showed that a first-order difference operator is analogous to enforcing a model covariance matrix (Oliver, 1994). For instance, an exponential model covariance function of the form

$$
C\left(z-z^{\prime}\right)=\sigma^{2} \exp \left(-\frac{\left|z-z^{\prime}\right|}{L}\right)
$$

can be enforced by minimizing the Sobolev functional norm

$$
\|f\|^{2}=\frac{1}{2 \sigma^{2}}\left[\frac{1}{L} \int d z f(z)^{2}+L \int d z\left(\frac{d f}{d z}\right)^{2}\right],
$$

where $L$ is a measure of correlation length between different locations. The variables $z$ and $z^{\prime}$ in equation (3.A.8) represent the spatial location of two discretization blocks, $\sigma$ denotes the variance of the reservoir parameters, and $f$ is the parameter to be reconstructed (e.g., porosity and/or permeability). The regularization parameter enforces a trade-off between the data misfit and the enforced model structure. If the length $L$ is too long, then the inverted 
parameter will tend to be very flat. If it is too small, then the role of the regularization function is negligible thereby leading to excessively oscillatory inverted parameters. Without any a-priori information about the length of spatial correlation, we make use of a large $L$ to control the change of model parameters.

\section{A.2.4. Rock Physics/Fluid Substitution Model}

As mentioned earlier, instead of elastic parameters, our goal is to estimate block porosity and permeability values. However, seismic waveform data are not explicitly related to porosity. A rock physics/fluid substitution model allows one to establish a quantitative link between fluid-flow petrophysical parameters and effective elastic properties. The basic equations for $\mathrm{P}$-wave and $\mathrm{S}$-wave velocities in isotropic, elastic non-porous media are given by

$$
v_{p}=\sqrt{\frac{K+\frac{4}{3} \mu}{\rho}},
$$

and

$$
v_{s}=\sqrt{\frac{\mu}{\rho}},
$$

where $K$ is the rock's bulk modulus (in turn a function of porosity, $\phi$, of water saturation, $S_{w}$, and of the mechanical properties of the rock's solid skeleton), $\mu$ is shear modulus, and $\rho$ is bulk density. The bulk density can be written as

$$
\rho=\phi S_{w} \rho_{w}+\phi\left(1-S_{w}\right) \rho_{o}+(1-\phi) \rho_{s},
$$

where $\rho_{\mathrm{w}}, \rho_{\mathrm{o}}$, and $\rho_{\mathrm{s}}$ are the densities of water, oil, and of the rock's solid skeleton, respectively. 
For simplicity, in this paper we assume a fluid model consisting of only water and oil. The rock's bulk modulus K of the saturated rock can be estimated from Gassmann's equation (Nur and Wang, 1998), namely,

$$
K=\left(K_{b}+\frac{4}{3} \mu\right)+\frac{\left(1-K_{b} / K_{s}\right)^{2}}{\left(1-\phi-K_{b} / K_{s}\right)\left(1 / K_{s}\right)+\phi / K_{f}},
$$

where the subscripts $b, s$, and $f$ stand for bulk, solid and fluid, respectively. We remark that Gassmann's equation makes three basic assumptions: First, the rock is homogeneous, and isotropic. Second, the pore space is completely connected. Third, the Gassmann's equation is valid only at low frequencies $(<100 \mathrm{~Hz})$. The porosity, elastic properties, and water saturation relationships summarized by equations (3.A.10) through (3.A.13) quantitatively link the wave equation with the two-phase fluid-flow equation. Because of this, in principle, the porosity distribution can be estimated by jointly inverting time records of fluid production measurements and pre-stack seismic data. The sensitivity of pre-stack seismic data to total porosity can be readily derived from the chain rule once the Fréchet derivatives of seismic data are obtained with respect to P-wave velocity, S-wave velocity, and bulk density. In this study, the derivatives of pre-stack seismic data with respect to P-wave velocity, S-wave velocity, and bulk density are computed numerically using finite differences.

\section{A.2.5. Numerical Studies of Feasibility}

\section{A.2.5.1. Synthetic Reservoir Model}

The synthetic reservoir model, displayed in Figure 3.A.1, consists of spatially heterogeneous, hydrocarbon-bearing sands embedded in a shale background. Spatial 
dimensions of the reservoir are $1260 \mathrm{ft} \times 1260 \mathrm{ft} \times 900 \mathrm{ft}$ in the $\mathrm{x}, \mathrm{y}$, and $\mathrm{z}$ directions, respectively. The model is discretized with a set of $21 \times 21 \times 30$ cubic blocks in the $x, y$, and $z$ directions, respectively. Block sizes are uniform in each direction and equal to $60 \mathrm{ft}$. Reservoir thickness is assumed uniform and equal to $60 \mathrm{ft}$. Each block is defined with specific values of permeability and porosity. Block permeabilities are homogeneous, yet vertically anisotropic, with the vertical permeability assumed to be one-tenth of the horizontal permeability. For the case of permeability, the inversion is posed to yield estimates of horizontal permeability; the corresponding vertical permeability is equal to one tenth of the horizontal permeability. Therefore, in this paper the nomenclature permeability and horizontal permeability are used without distinction.

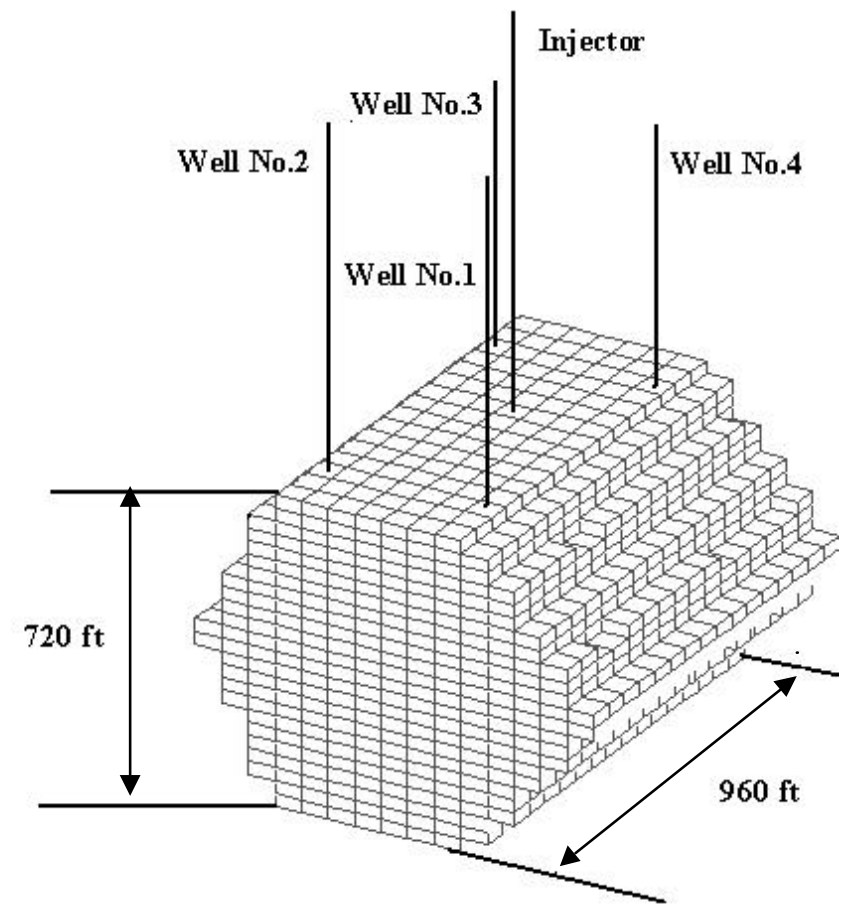

Figure 3.A.1. Graphical description of the synthetic 3D reservoir model. Well locations are indicated with vertical lines penetrating through the reservoir. The injection well is located close to the center of the reservoir. There are four production wells symmetrically located around the injection well. 
A five-spot well pattern is used to sweep the hydrocarbons saturating the reservoir volume. The central well is used for water injection while the remaining wells are dedicated to oil production. Well locations are shown in Figure 3.A.2. The injector well is located in the center of the block $[x, y]=[13,11]$ and four producers, numbered 1 through 4 , are located in the center of the blocks $[10,6],[16,6],[16,16]$, and $[10,16]$. The total flow rate at each producer is fixed at $900 \mathrm{STB} / \mathrm{D}$ and the flow rate at the injector is fixed at $400 \mathrm{STB} / \mathrm{D}$. Twophase relative permeability and capillary pressure curves are employed in the forward modeling of fluid-flow measurements and these are shown in Figures 3.A.3 and 3.A.4, respectively. Relative permeability and capillary pressure curves are assumed constant for all the discretization blocks.

Spatial distributions of permeability and porosity for the discretization blocks in the synthetic 3D reservoir model are assigned by means of geostatistical simulation. In so doing, the mean of the log-permeability is assumed to be 4.0 with a variance equal to 0.2 . The mean of the porosity is assumed to be 0.2 with a variance equal to 0.0004 . Logarithmic permeability and porosity are assigned a spatial variogram range equal to $600 \mathrm{ft}$. Each layer is assumed to exhibit a spherical variogram model. No correlation is assumed between the porosity and log-permeability. Block permeabilities and porosities are generated using unconditional simulation computed from Cholesky decomposition of the covariance matrix and are shown in Figures 3.A.5 and 3.A.6, respectively. Measurements input to the inversions are simulated numerically from the geostatistically generated permeability and porosity models. Errors in the measurements are assumed independent. Details of the reservoir fluid and rock properties together with the characteristics of the reservoir's spatial architecture are described in Table 3.A.1. 
Fluid production measurements were simulated from the time interval between 500 and 1000 days after the onset of water injection. During the simulation time, water breakthrough occurs after 600 days. In all cases, synthetically generated water-oil ratio measurements were contaminated with additive random Gaussian noise. The standard deviation of the noise was assumed equal to $5 \%$ of the observed data.

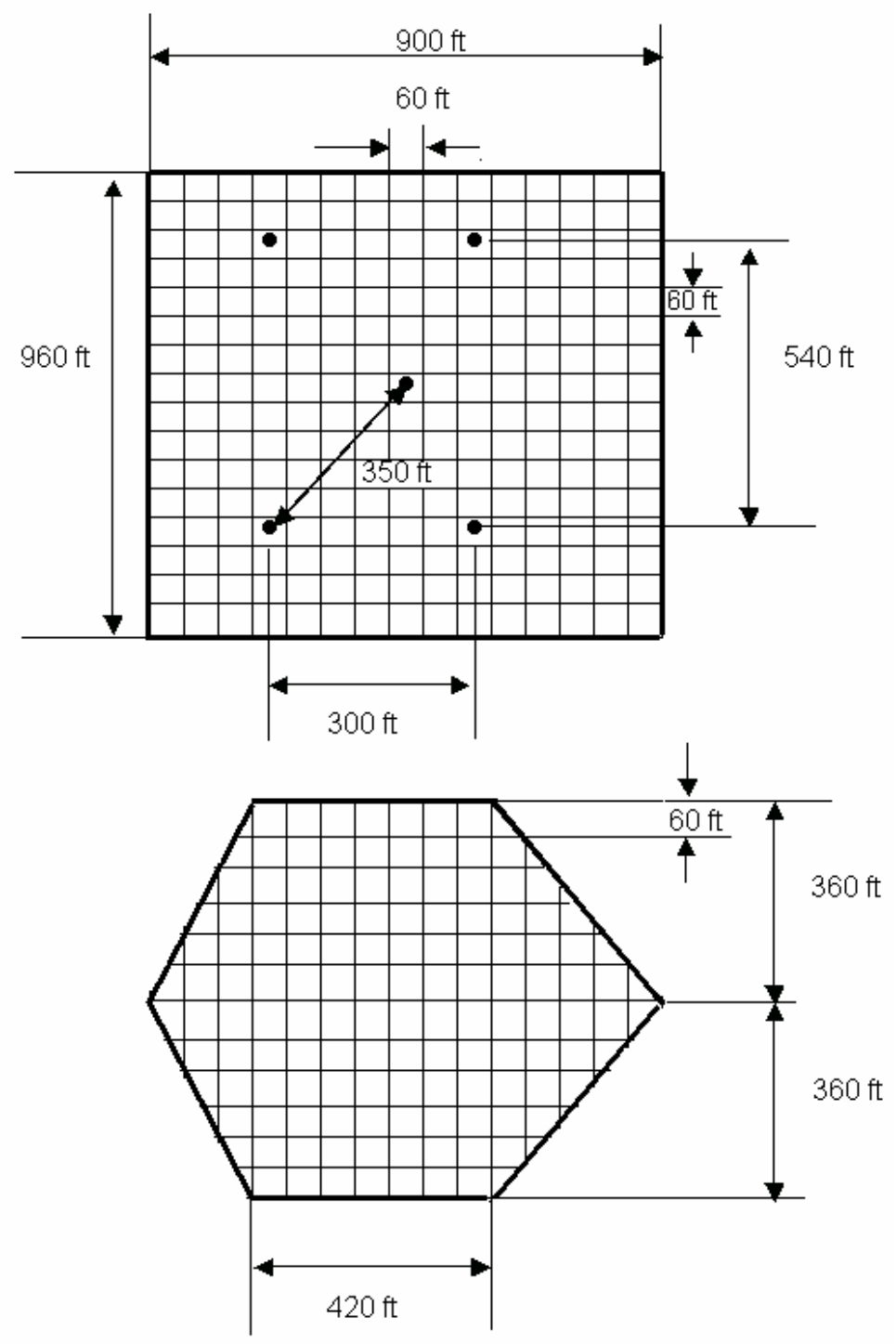

Figure 3.A.2. Plan view and cross section of the reservoir model shown in Figure 3.A.1. The plan view indicates locations of the injector and producer wells together with the geometrical dimensions of the reservoir. A regular Cartesian grid inserted within the reservoir indicates the location, dimensions, and number of discretization blocks used to describe the spatial dis tribution of reservoir properties. Vertical dimensions of the reservoir and of the discretization blocks are shown om the cross-section. 


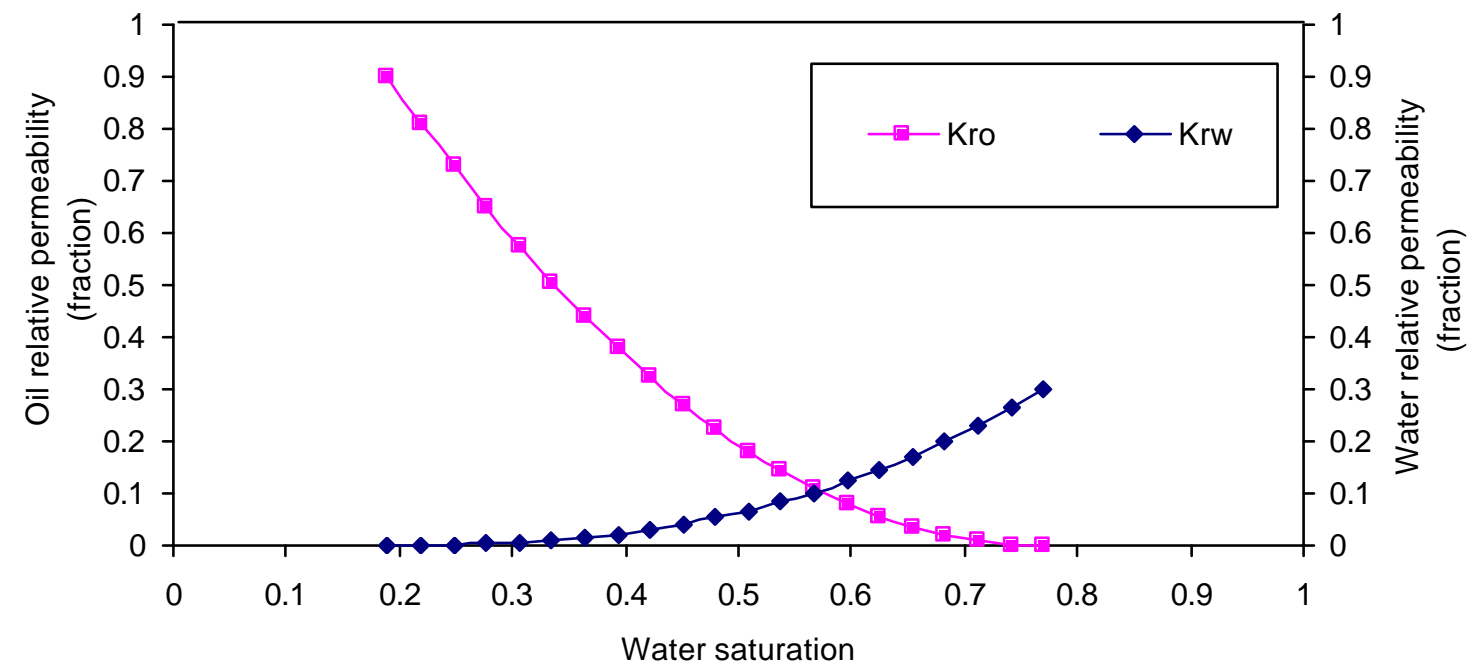

Figure 3.A.3. Water-oil relative permeability curves used in the numerical simulation of the water-oil system.

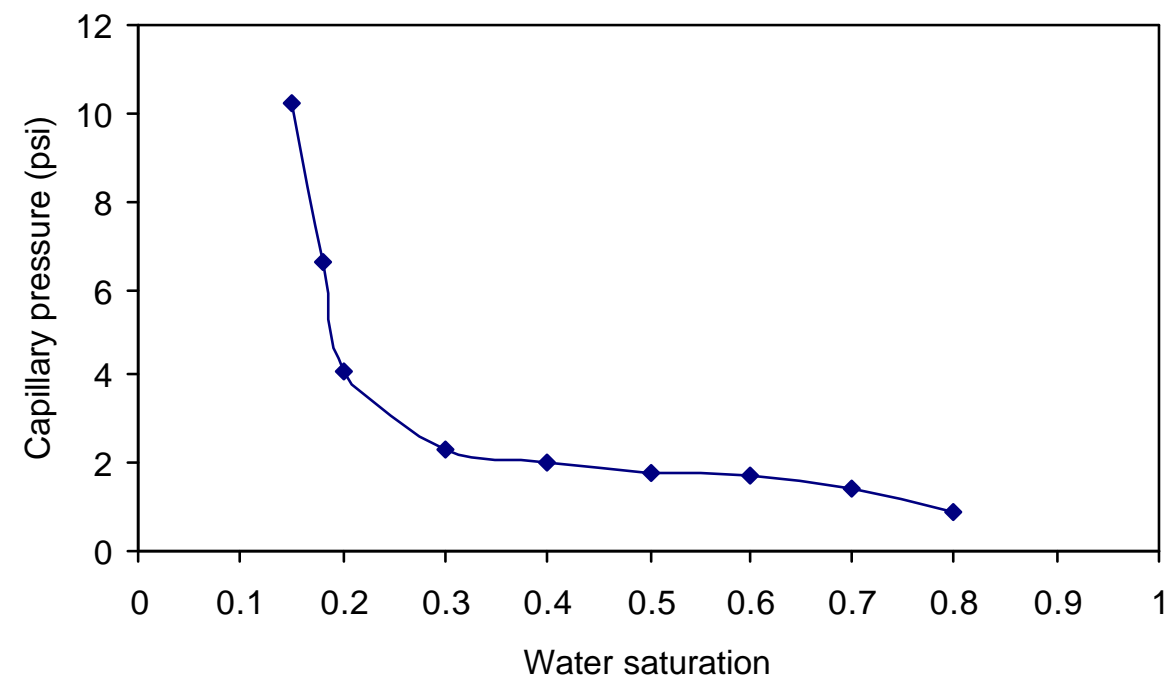

Figure 3.A.4. Capillary pressure curve used in the numerical simulation of the water-oil system. 


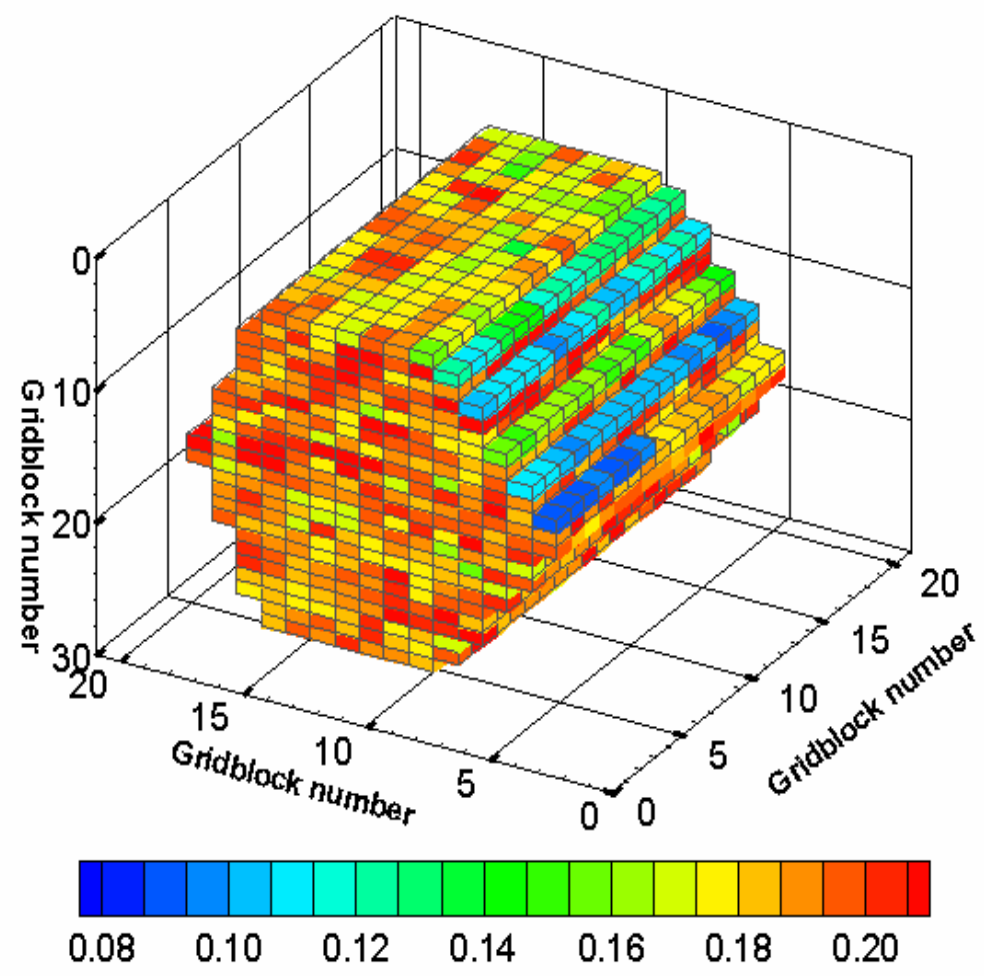

Figure 3.A.5. Graph depiction of the true spatial distribution of porosity.
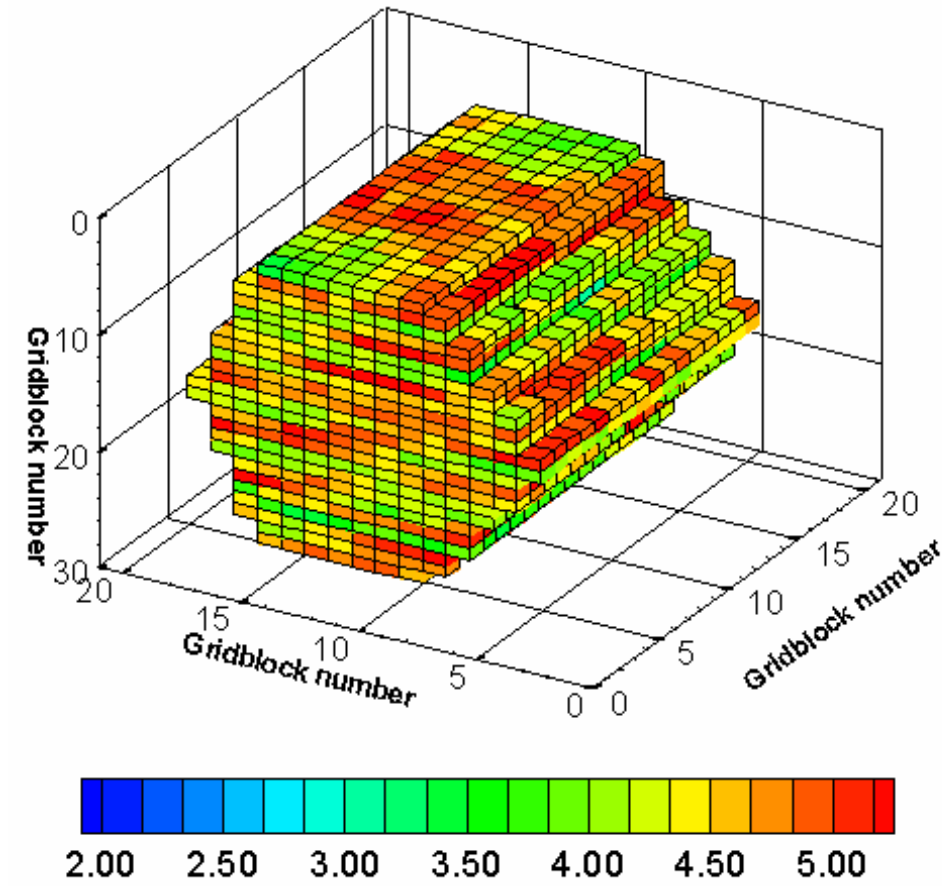

Figure 3.A.6. Graph depiction of the true spatial distribution of log-permeability [log (md)]. 


\begin{tabular}{|c|c|c|}
\hline \multicolumn{3}{|c|}{$\begin{array}{l}\text { Table 3.A.1. Summary of fluid and reservoir properties used to } \\
\text { construct the synthetic reservoir model and to numerically simulate } \\
\text { time records of fluid production data }\end{array}$} \\
\hline & Property & $\begin{array}{l}\text { Values } \\
\end{array}$ \\
\hline \multirow{8}{*}{ Fluid } & Water density & $62.40 \mathrm{lb} / \mathrm{ft}^{3}$ \\
\hline & Oil density & $52.88 \mathrm{lb} / \mathrm{ft}^{3}$ \\
\hline & Water viscosity & $1.00 \mathrm{cp}$ \\
\hline & Oil viscosity & $0.92 \mathrm{cp}$ \\
\hline & Water compressibility & $3.20 \times 10^{-6} \mathrm{psi}^{-1}$ \\
\hline & Oil compressibility & $1.00 \times 10^{-5} \mathrm{psi}^{-1}$ \\
\hline & Water formation volume factor & $1.00 \mathrm{RB} / \mathrm{STB}$ \\
\hline & Oil formation volume factor & $1.16 \mathrm{RB} / \mathrm{STB}$ \\
\hline \multirow{8}{*}{ Reservoir } & Initial water saturation & 0.19 \\
\hline & Residual oil saturation & 0.23 \\
\hline & Porosity & $\begin{array}{l}\text { Gaussian probability } \\
\text { distribution with mean } 0.19 \\
\text { and standard deviation } \\
0.020\end{array}$ \\
\hline & Permeability & $\begin{array}{c}\text { Gaussian probability } \\
\text { distribution with log - mean } \\
4.0 \text { and standard deviation } \\
0.45 \\
\end{array}$ \\
\hline & $\begin{array}{l}k_{r w}^{o} \text { end point of water relative } \\
\text { permeability }\end{array}$ & 0.20 \\
\hline & $\begin{array}{l}k_{r o}^{o} \text { end point of oil relative } \\
\text { permeability }\end{array}$ & 0.90 \\
\hline & $\begin{array}{l}k_{x} / k_{y} \text { ratio of two principal } \\
\text { permeability directions }\end{array}$ & 1.00 \\
\hline & $\begin{array}{l}k_{x} / k_{z} \text { ratio of horizontal } \\
\text { permeability and vertical } \\
\text { permeability }\end{array}$ & 0.10 \\
\hline \multirow{8}{*}{ Simulation } & Number of gridblocks & $21 \times 21 \times 30$ \\
\hline & Gridblock size & $60 \times 60 \times 30 \mathrm{ft}$ \\
\hline & Injection rate & $900 \mathrm{STB} / \mathrm{D}$ \\
\hline & Production rate & $4000 \mathrm{STB} / \mathrm{D}$ \\
\hline & Perforation & All layers \\
\hline & Number of production wells & 4 \\
\hline & Number of injection wells & 1 \\
\hline & $\begin{array}{l}\text { Distance between injector and } \\
\text { producers }\end{array}$ & $350 \mathrm{ft}$ \\
\hline
\end{tabular}

\section{A.2.5.2. Inversion of Time Records of Fluid Production Data}

In the following numerical example, we investigate the use of water-oil ratio (WOR)

measurements for the quantitative estimation of spatial distributions of permeability and

porosity. In general, WOR data constitute a relatively late-time measurement in the produc- 
tion life of a reservoir. Until the water breakthrough occurs in one or more production wells WOR information remains nonexistent. After water breakthrough, the ratio of the volumetric production rates of water and hydrocarbon components is measured at surface conditions. Hence, in this paper we focus on conventional WOR measurements acquired on a well-towell basis.

As emphasized earlier, history matching is essentially a non-unique process. Traditionally, non-uniqueness can be mitigated by incorporating a-priori information into the inversion. The a-priori information could be well-log data, rock-core data, or geological information. Mathe matically, statistical correlation properties of reservoir parameters can be imposed through the use of a model covariance matrix, $C_{m}$, and by initializing the inversion with an educated model, $m_{o}$. Without any a-priori information about porosity and permeability, we make use of a first-order differential regularization strategy to impose a degree of smoothness of the unknown spatial distributions of porosity and permeability. For the example cases considered in this paper, the inversion was initialized with uniform values of porosity and permeability for all of the discretization blocks. As can be observed in Figures 3.A.7 and 3.A.8, the inverted distributions of porosity and permeability do not compare well with the true distributions even though the production data are matched within 5\% (see Figures 3.A.9-3.A.12). Note that significant spatial variations in the inverted parameters occur only near the wells. The inverted distributions are highly influenced by the actual values of porosity and permeability in the vicinity of wells since the fluid flow rates are extremely sensitive to these block parameters (Wu et al., 1999). Poor reconstructions of the spatial distributions of permeability and porosity, together with a good match of WOR measurements, clearly indicate the high level of non- uniqueness in the inversion of WOR 
data. It must be pointed out that, due to the non-uniqueness of the inverse problem, the choice of parameter covariance matrix heavily influences the final inverted model. Without a-priori information, the selection of the degree of model smoothness remains strictly subjective. In this paper, the choice of a model covariance matrix is driven by the subjective choice of a smooth spatial solution while the production data are matched. Accordingly, we assumed a lateral correlation length equal to ten discretization blocks and a vertical correlation length equal to one discretization block.

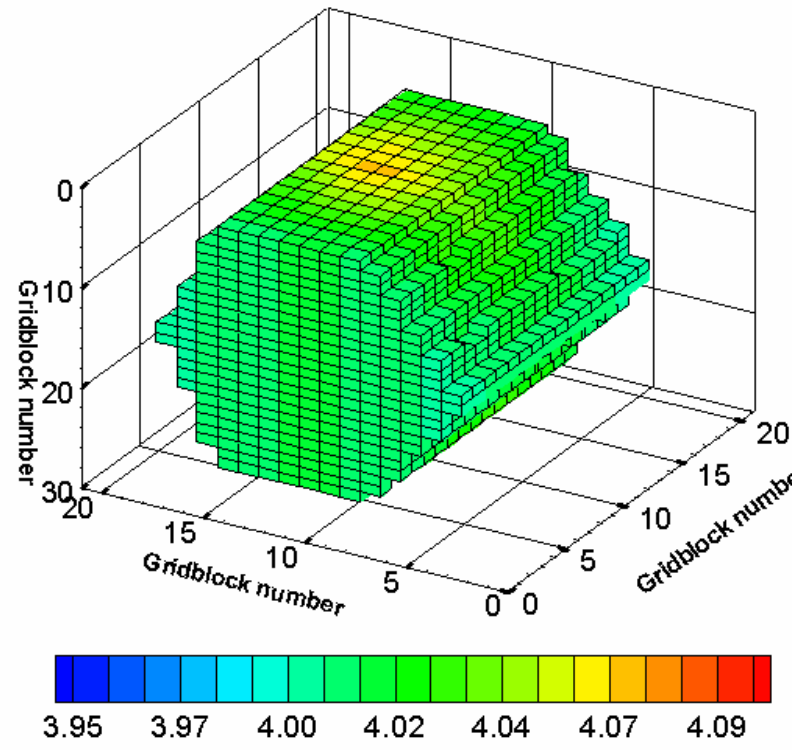

Figure 3.A.7. Graphical description of the spatial distribution of log-permeability estimated from the inversion of time records of water-oil ratio. [log (md)]. 

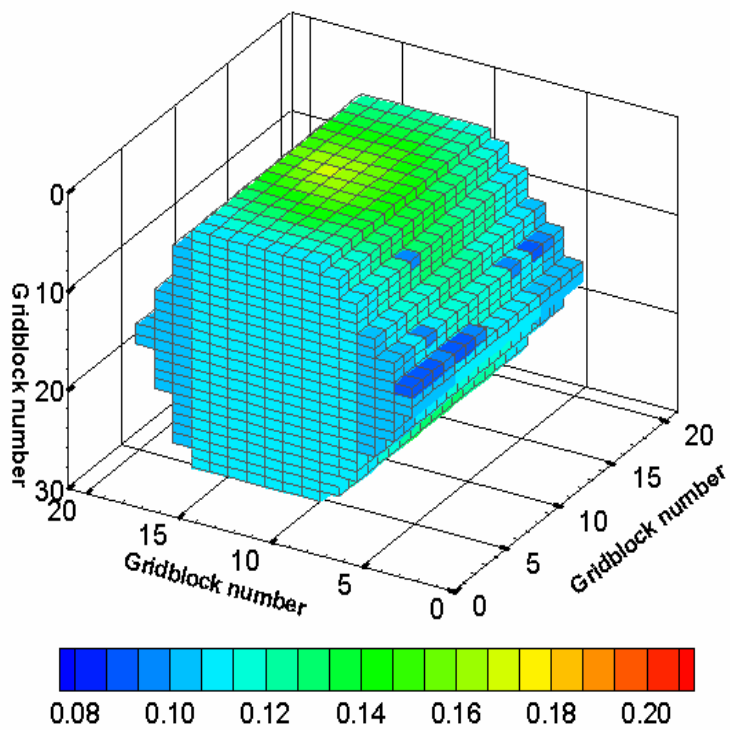

Figure 3.A.8. Graphical description of the spatial distribution of porosity estimated from the inversion of time records of water-oil ratio.

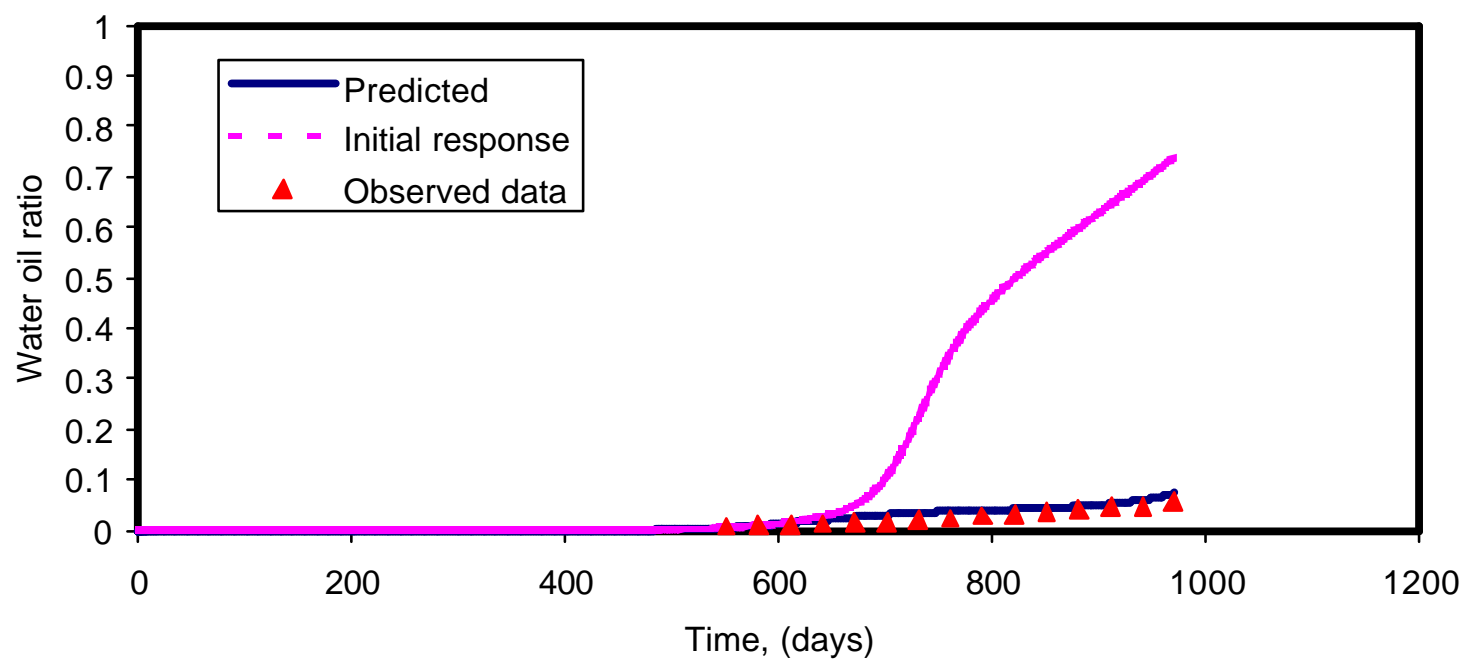

Figure 3.A.9. Comparison of the measured and numerically simulated time record of wateroil ratio (WOR) for the production Well No. 1. The measured data were contaminated with $5 \%$ additive Gaussian random noise. 


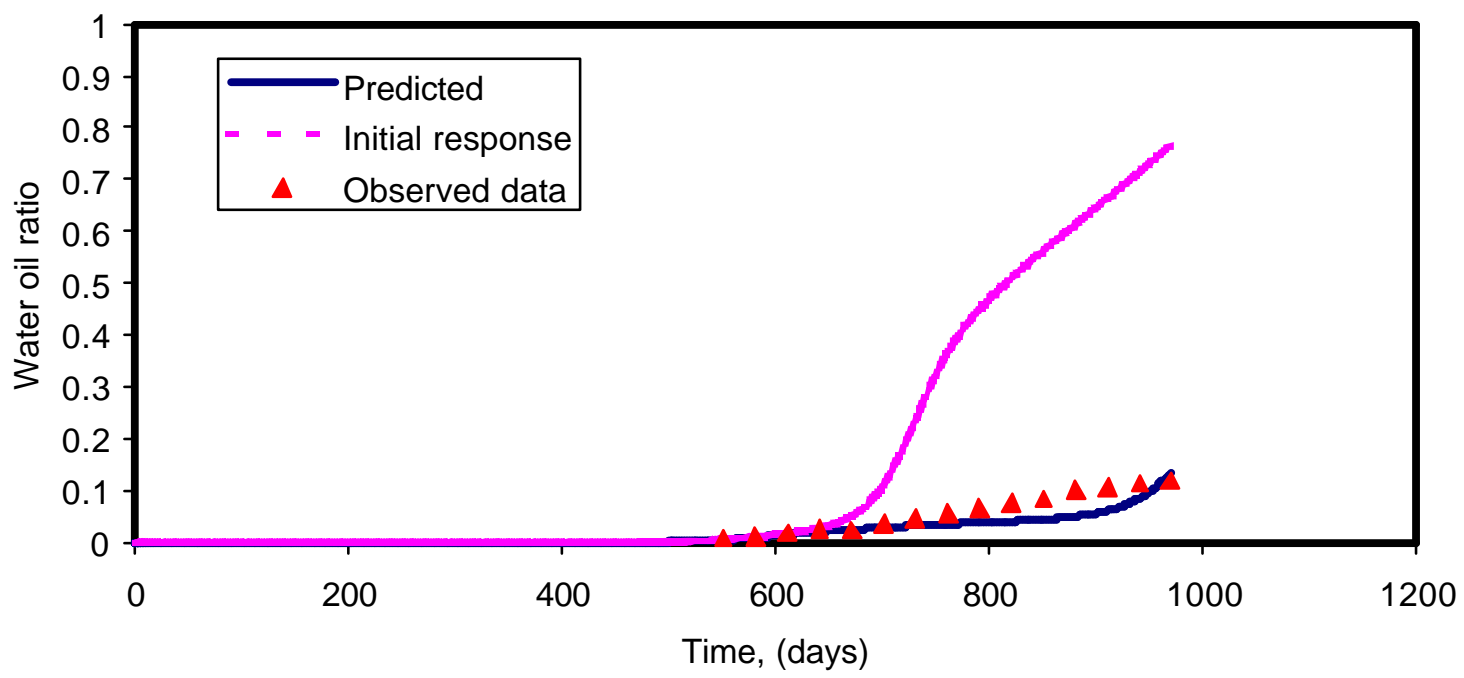

Figure 3.A.10. Comparison of the measured and numerically simulated time record of wateroil ratio (WOR) for the production well No. 2. The measured data were contaminated with $5 \%$ additive Gaussian random noise.

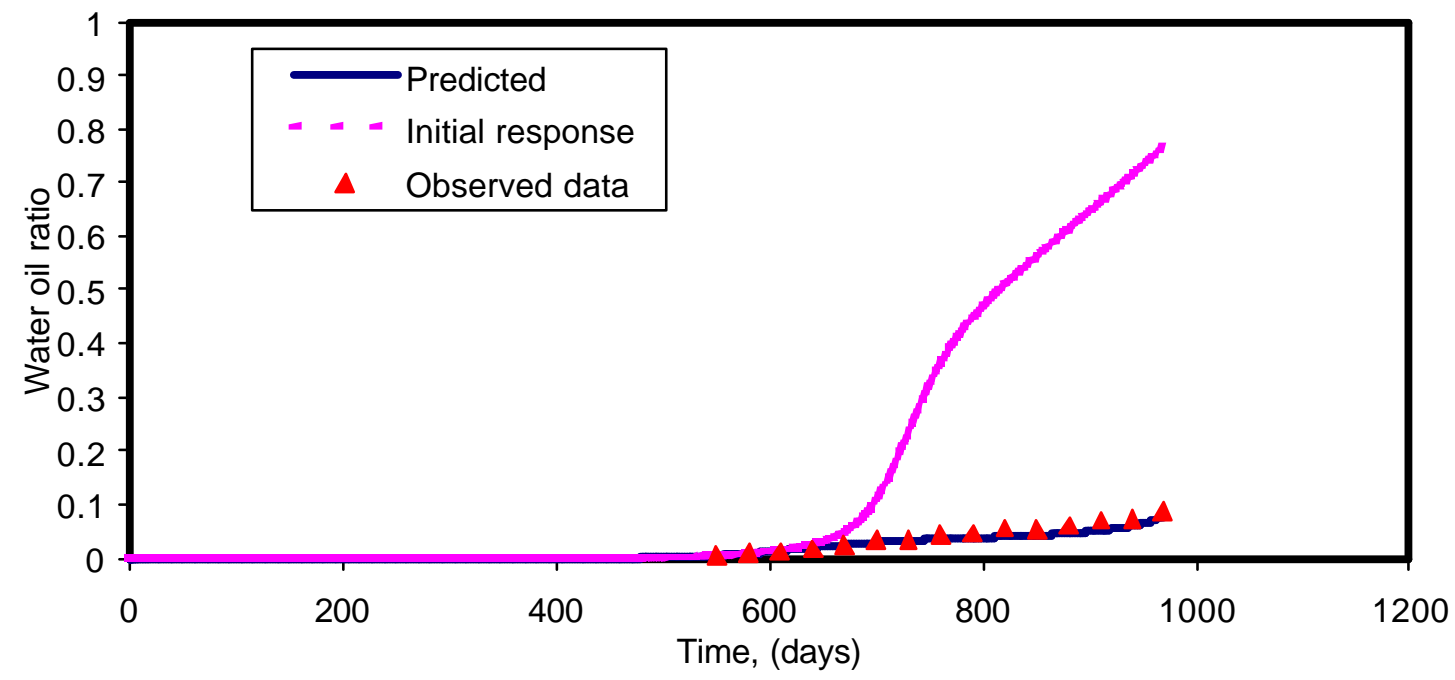

Figure 3.A.11. Comparison of the measured and numerically simulated time record of wateroil ratio (WOR) for the production well No. 3. The measured data were contaminated with $5 \%$ additive Gaussian random noise. 


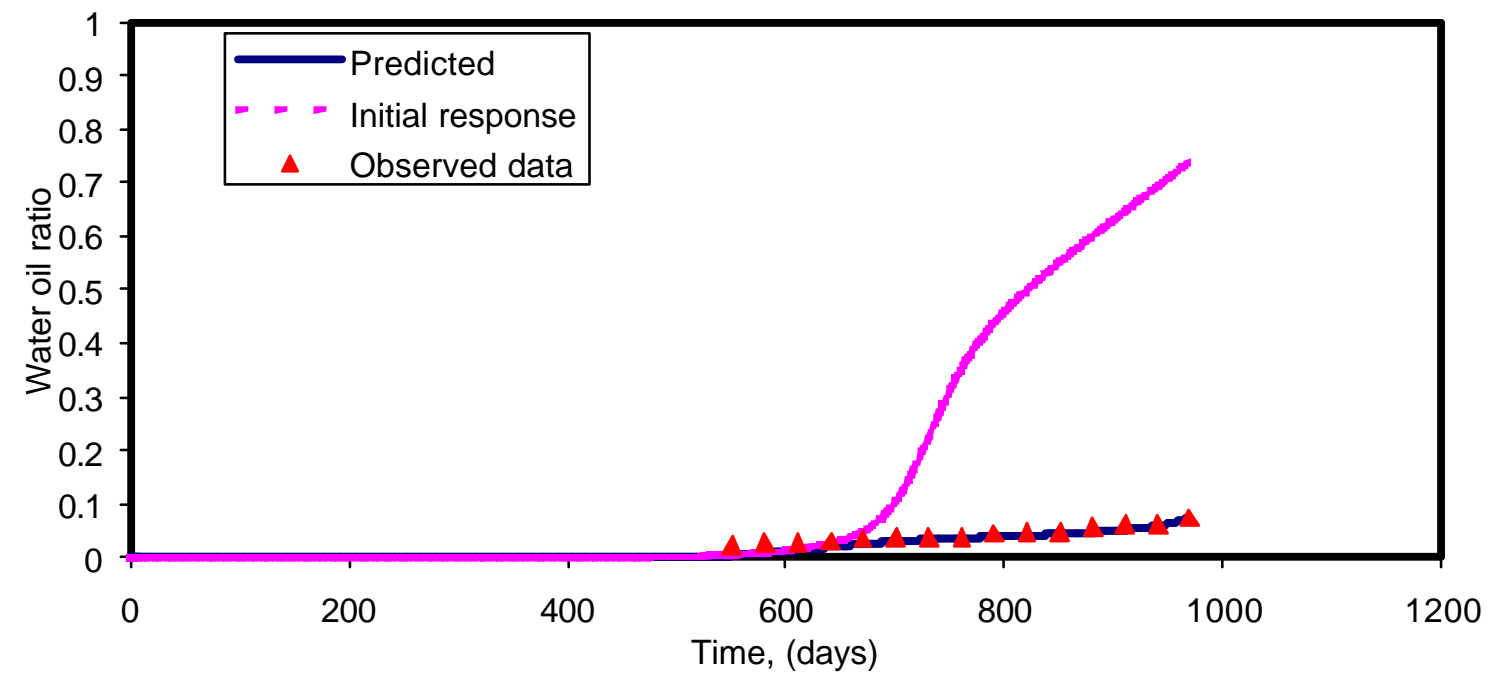

Figure 3.A.12. Comparison of the measured and numerically simulated time record of wateroil ratio (WOR) for the production well No. 4. The measured data were contaminated with $5 \%$ additive Gaussian random noise.

\section{A.2.5.3. Synthetic Elastic Model}

The second test example was designed to appraise the sensitivity of pre-stack seismic data to the spatial distribution of porosity. Petrophysical parameters were transformed into corresponding block elastic parameters using the Gassmann's effective medium equations. Seismic data were simulated as locally 1D pre-stack gathers. Accordingly, the synthetic subsurface model consists of a 900-ft thick sand layer embedded in a background shale with the top interface between shale and sand located at a depth of $4000 \mathrm{ft}$. Simulation of seismic data was performed assuming 10 source-receive offsets with a uniform receiver spacing equal to $1260 \mathrm{ft}$ and a constant time sampling rate of $2 \mathrm{~ms}$ in the interval from 0 to 1.64 seconds. Pre-stack gathers were assumed to include source-receiver angles from 4.5 to 45 degrees. The synthetic seismograms were generated assuming a zero-phase Ricker wavelet of central frequency equal to $35 \mathrm{~Hz}$. All the synthetic pre-stack seismic data were corrected for normal moveout. 
Figure 3.A.13 shows the Ricker wavelet used in this study. Zero-mean Gaussian random noise of standard derivation equal to $5 \%$ of the seismic waveform amplitude was added to the simulated pre-stack seismic data. The noisy data were inverted using the new data subspace algorithm discussed earlier. Details of the assumed petrophysical properties and of the acquisition parameters for the seismic data are described in Tables 3.A.2 and 3.A.3, respectively.

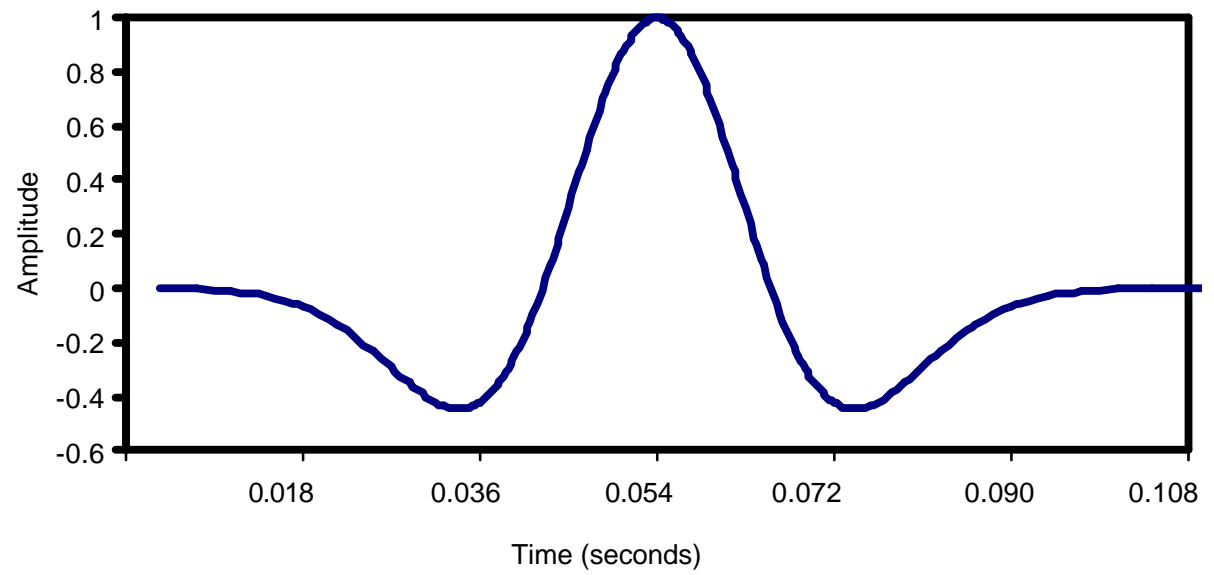

Figure 3.A.13. Graphic representation of the Ricker wavelet used in the simulation of prestack seismic data.

\begin{tabular}{|l|c|c|c|}
\hline \multicolumn{4}{|c|}{ Table 3.A.2. Descriptions of the bulk modulus, shear modulus, } \\
and bulk density of the rock and fluid constituents assumed in the \\
construction of the synthetic reservoir model.
\end{tabular}




\begin{tabular}{|c|c|c|c|}
\hline Properties & Values & Properties & Values \\
\hline Wavelet type & $\begin{array}{l}\text { Zero-phase Ricker } \\
\text { wavelet }\end{array}$ & Number of samples & 1024 \\
\hline Central frequency & $35 \mathrm{~Hz}$ & Simulation time & $2 \mathrm{~s}$ \\
\hline Sampling interval & $0.002 \mathrm{~s}$ & Number of sand layers & 30 \\
\hline $\begin{array}{l}\text { Number of offsets at } \\
\text { each location }\end{array}$ & 10 & $\begin{array}{c}\text { Number of shale } \\
\text { layers }\end{array}$ & 50 \\
\hline Angle intervals & 4.5 degree & $\begin{array}{c}\text { Thickness of each } \\
\text { sand layer }\end{array}$ & $30 \mathrm{ft}$ \\
\hline $\begin{array}{l}\text { Distance between } \\
\text { two adjacent offsets }\end{array}$ & $49 \mathrm{ft}$ & $\begin{array}{c}\text { Thickness of each } \\
\text { shale layer }\end{array}$ & $80 \mathrm{ft}$ \\
\hline
\end{tabular}

\section{A.2.5.4. Inversion of Pre-stack Seismic Data}

We assume that the petrophysical parameters of the shale layers are known. The thirty layers comprising the sand zone located between $4000 \mathrm{ft}$. and $4900 \mathrm{ft}$. are the target layers of unknown elastic and petrophysical properties. Local 1D synthetic seismograms were generated for the 80-layer model. Inversions were conducted using the ten traces simulated from local 1D models. The 3D model is built as a "mosaique" of the sequential inversion of local 1D models. A model covariance matrix, $C_{m}$, was enforced by the inversion with entries calculated directly from equation (3.A.8) assuming that $\sigma^{2}$ in that equation is equal to actual variance of porosity used to construct the synthetic reservoir model. The data covariance matrix, $C_{D}$, on the other hand, was constructed as a diagonal matrix with positive entries. Unlike standard approaches, where the entries of the diagonal of $C_{D}$ represent the variance of the measured data, here the entries of the diagonal are used as weighting factors that assign a relative importance to the various offsets included in an individual CMP gather. 
Figure 3.A.14 graphically describes the inversion results obtained with the data subspace method designed to invert the spatial distribution of porosity. The estimated spatial distribution of porosity successfully reproduces the main features of the original distribution in both the lateral and vertical directions. Recall that we assumed that a-priori information about porosity was not available and that the inversion was initialized with a uniform distribution (see Figure 3.A.15). Despite the lack of a-priori information about the spatial distribution of porosity, a very good reconstruction is attained by the inversion of pre-stack seismic data. Using histograms, one can further appraise the closeness between the distributions of inverted and true porosity. Figures 3.A.16 and 3.A.17 show histograms of the true porosity and of the porosity inverted from pre-stack seismic data. The two histograms are almost identical.

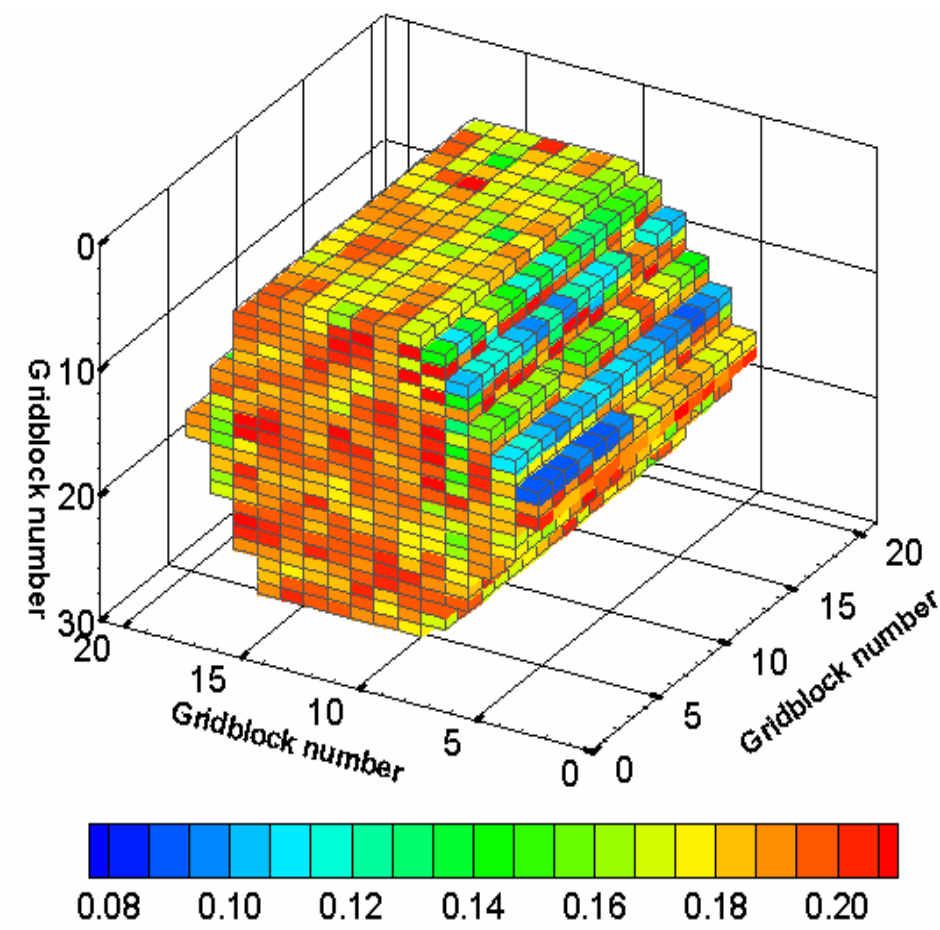

Figure 3.A.14. Graphic depiction of the spatial distribution of porosity inverted from prestack seismic data. 


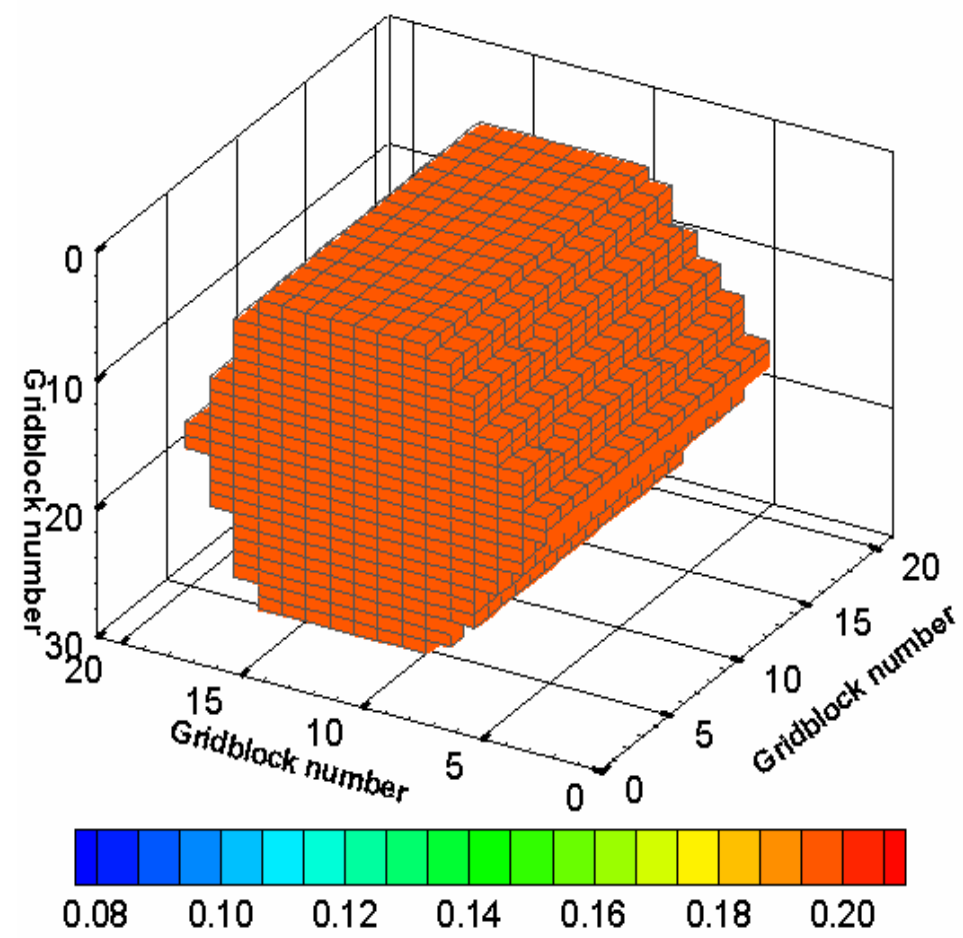

Figure 3.A.15. Graphic depiction of the spatial distribution of porosity used to initialize the inversion of pre-stack seismic data.

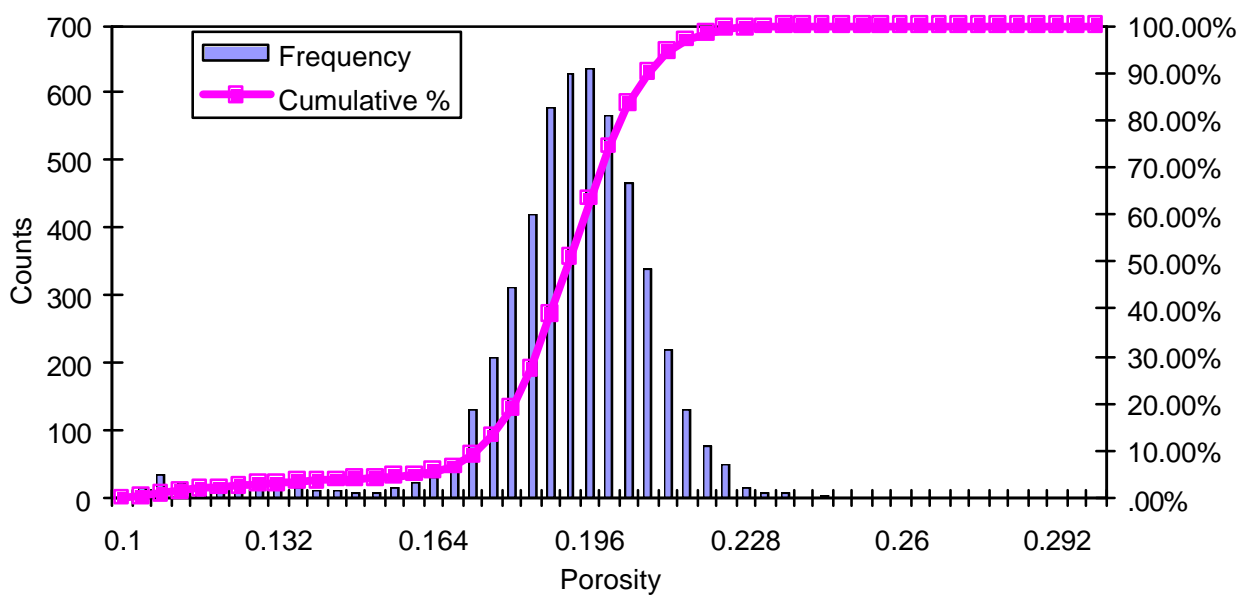

Figure 3.A.16. Graphic depiction of the statistical distribution of porosity values in the synthetic reservoir model. 


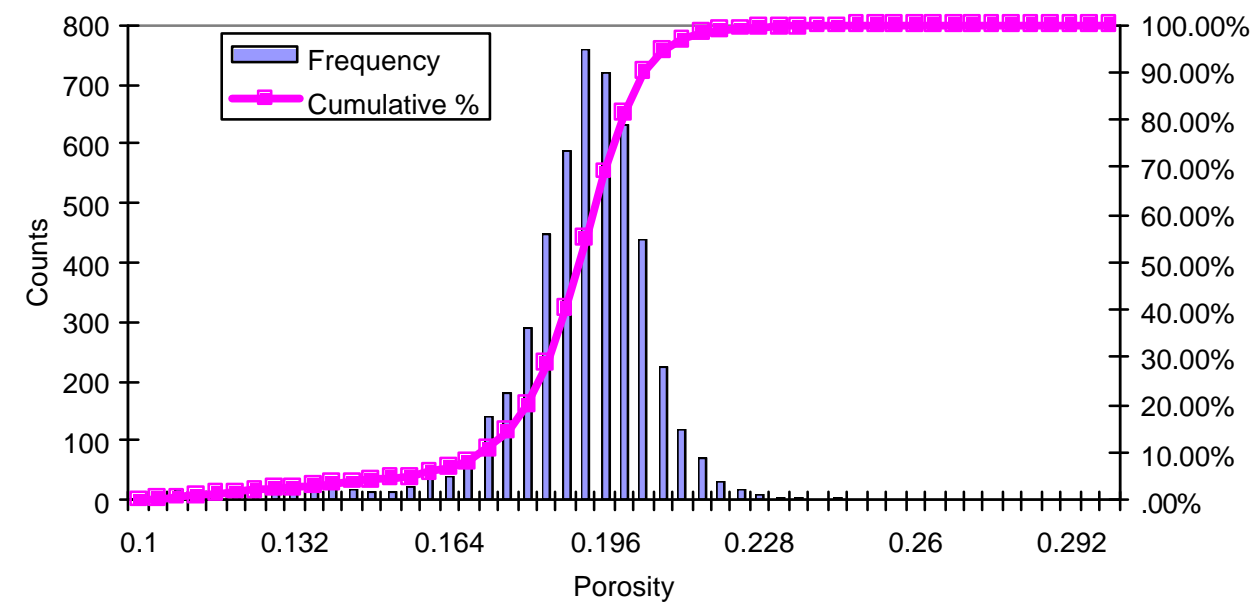

Figure 3.A.17. Graphic depiction of the statistical distribution of porosity values in the reservoir model inverted from pre-stack seismic data

In Figure 3.A.18, we show a comparison of the synthetic seismograms for a CMP gather, which were computed from the inverted porosity distribution as well as from the corresponding gather simulated from the true porosity distribution. The match between the two-waveform data sets is good even though the initial guess for porosity is that of a constant value. The fit of the seismic data achieved by the inversion can also be quantitatively assessed with the correlation coefficient between the observed data and the data simulated from the inverted model. The correlation coefficient varies between -1 and 1 . It is equal to 1 when there is a perfect match between the two data sets. Figures 3.A.19 and 3.A.20 display maps of correlation coefficient between the pre-stack seismic data simulated for the initial model and from the porosity model rendered by the inversion. As displayed in Figure 3.A.20, most of the correlation coefficients are above 0.90 after the inversion successfully matches the input pre-stack seismic waveform data. 
(a)
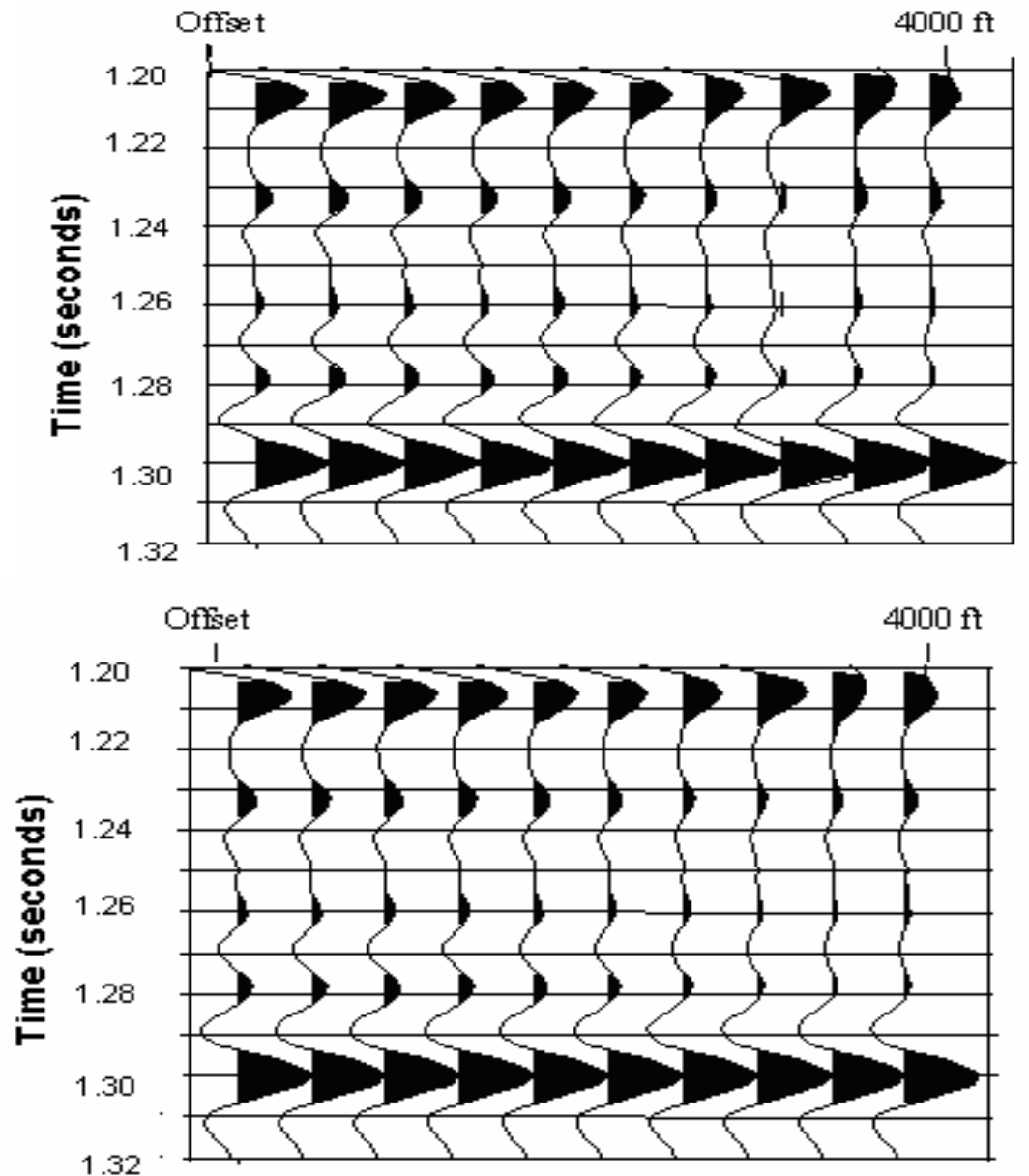

(b)

Figure 3.A.18. Comparison of the measured and simulated seismic traces for a single CMP gather: Panel (a) shows the measured seismic data and panel, (b) shows the numerical simulated seismic data. The measured data were contaminated with 5\% additive Gaussian random noise. 


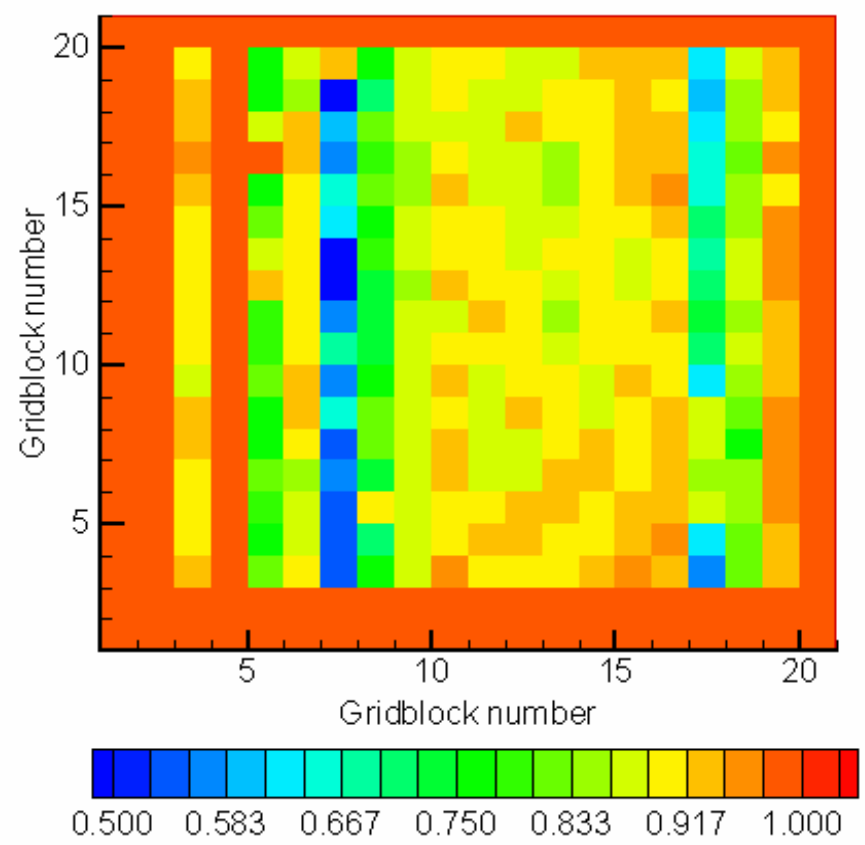

Figure 3.A.19. Plan view of the statistical correlation between the observed pre-stack seismic data and the pre-stack seismic data simulated from the uniform reservoir model used to initialize the inversion.

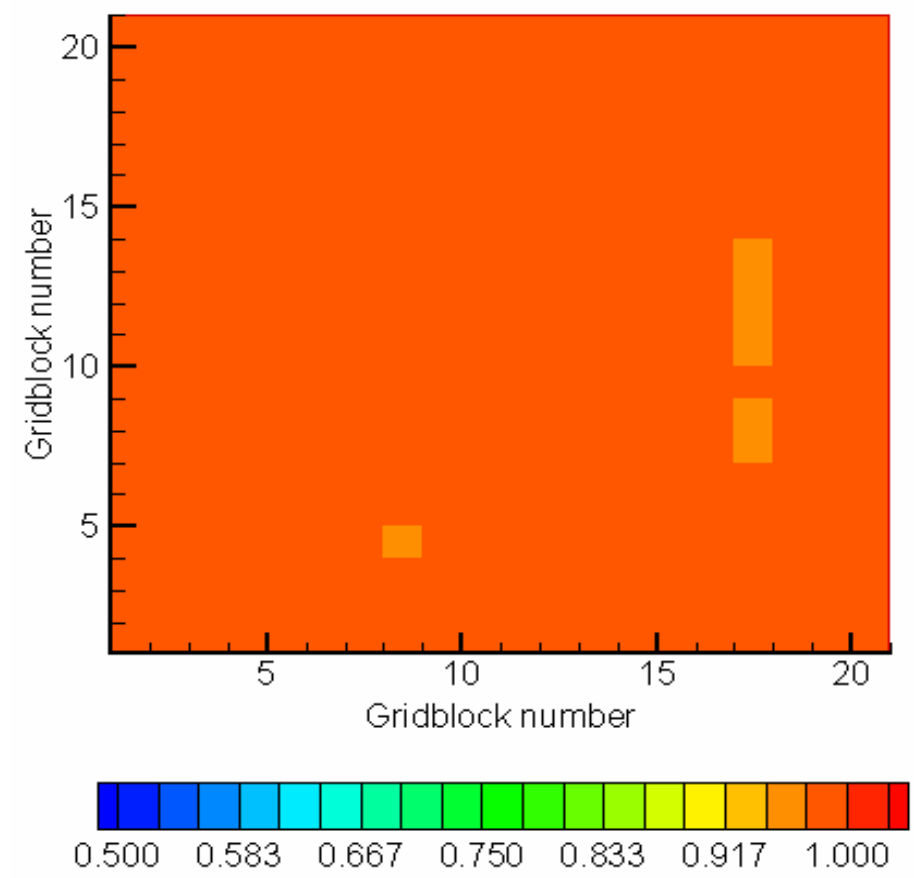

Figure 3.A.20. Plan view of the statistical correlation between the observed pre-stack seismic data and the pre-stack seismic data simulated from the inverted reservoir mode. 
Once the block porosities are inverted, P-wave and $\mathrm{S}$-wave velocities can be calculated using Gassmann's equation. Figure 3.A.21 is a composite plot of P- wave velocity obtained from the inversion of pre-stack seismic data. There are three panels in that figure. Panel (a) shows the vertical profile of P-wave velocity used to initialize the inversion, Panel (b) shows the profile of P-wave velocity rendered by the inversion of seismic waveform data, and Panel (c) shows the original profile of P-wave velocity. Figure 3.A.22 shows similar comparison panels for vertical profiles of S-wave velocity. In both cases, the reconstructed and original profiles exhibit a good visual correlation.

(a)

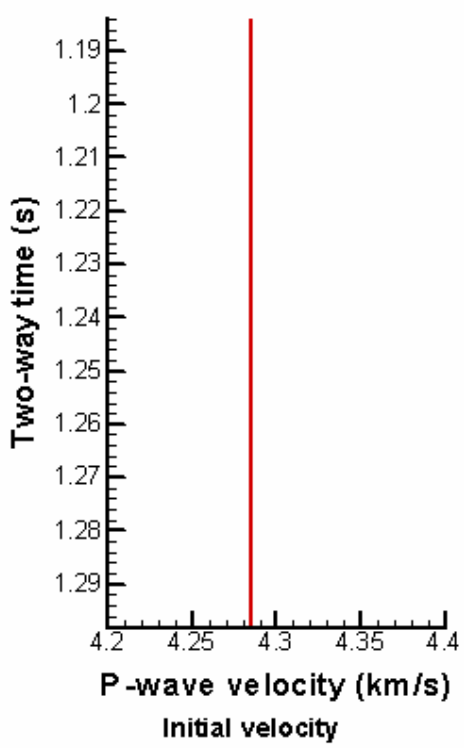

(b)

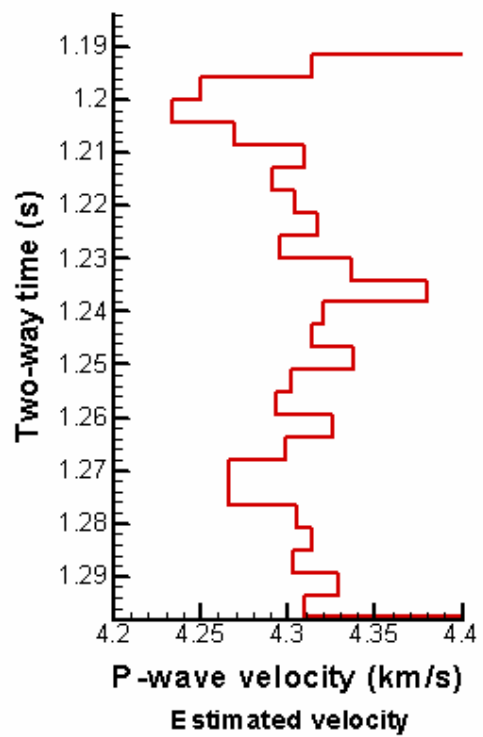

(c)

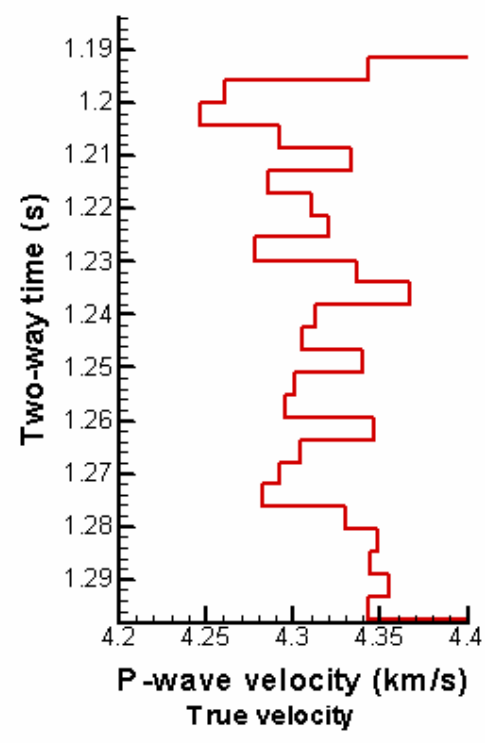

Figure 3.A.21. Vertical profiles of P-wave velocity: (a) Initial velocity, (b) true model, and (c) estimated model from waveform inversion 
(a)

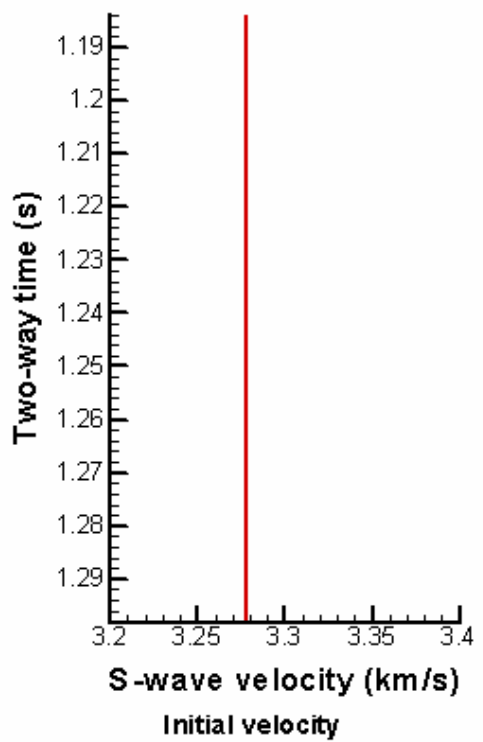

(b)

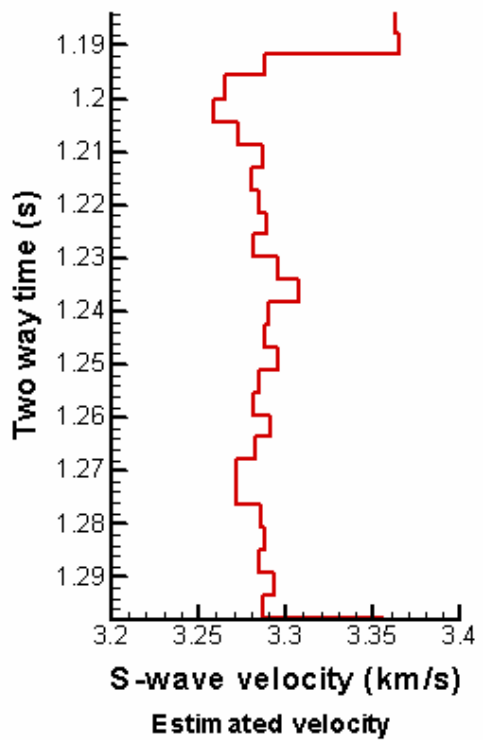

(c)

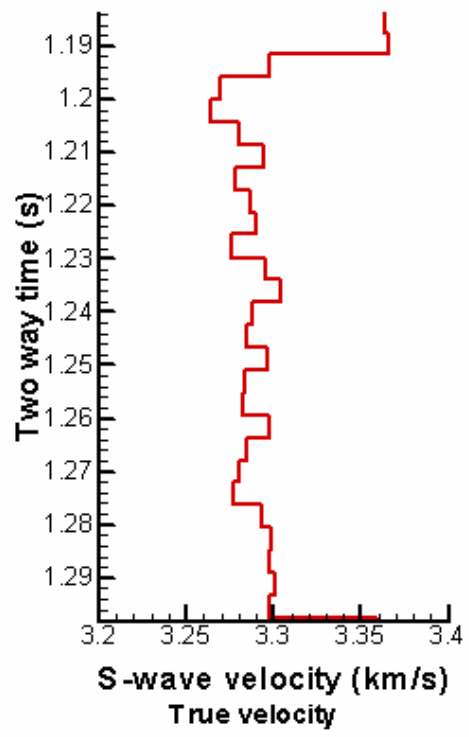

Figure 3.A.22. Vertical profiles of S-wave velocity: (a) Initial velocity, (b) true model, and (c) estimated model from waveform inversion.

The vertical resolution of pre-stack data is governed by the relative difference between the seismic wavelength and the layer thickness. Different central frequencies were used to assess the vertical resolution of pre-stack data to estimate porosity. Figure 3.A.23 shows the spatial distribution of porosity estimated from synthetic seismic data generated with a Ricker wavelet of central frequency equal to $20 \mathrm{~Hz}$. It is found that the vertical and lateral resolution of the inverted distribution of porosity are still high compared to the porosity distribution estimated from seismic data generated with a Ricker wavelet of central frequency equal to $35-\mathrm{Hz}$. 


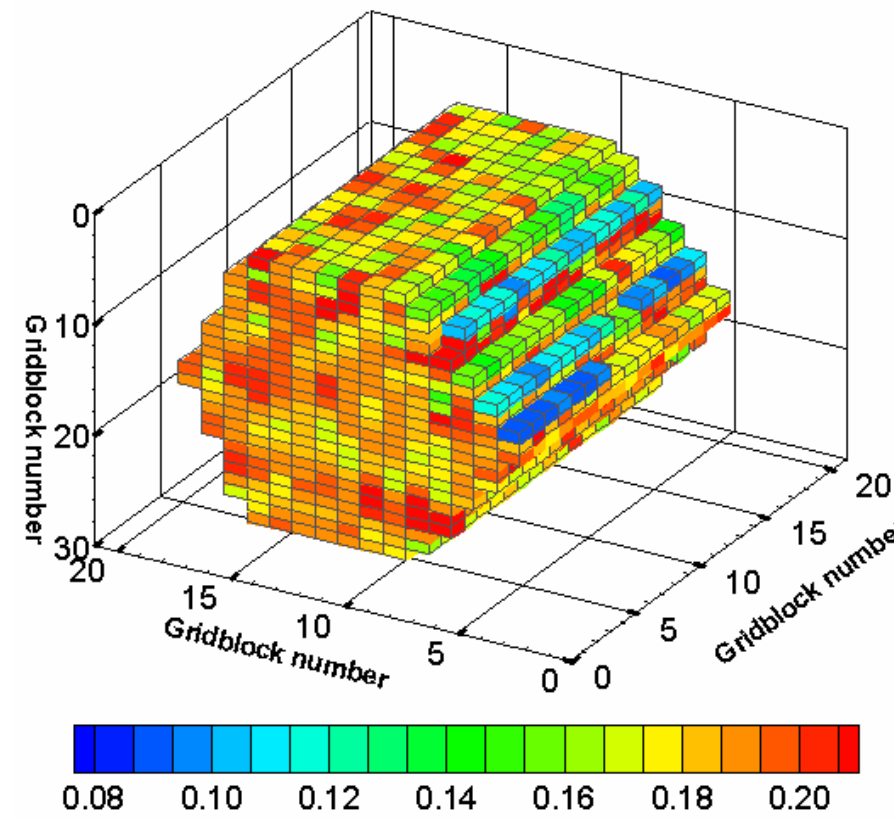

Figure 3.A.23. Graphic depiction of the spatial distribution of porosity inverted from prestack seismic data assuming a low frequency Ricker wavelet (central frequency=20 Hz).

In the previous models, porosity values used in Gassmann's equation for the calculation of P-wave and S-wave velocities exhibit a Gaussian distribution with a mean equal to 0.2 . Next we consider the inversion of a spatial distribution of porosity that exhibits a lower value of average porosity. Figure 3.A.24 shows the true porosity distribution. The mean of this porosity distribution is 0.08. A comparison between the true porosity distribution and the inverted porosity distribution (shown in Figure 3.A.25) reveals that the inverted porosity distribution does not properly reproduce the features of original porosity distribution. For completeness, Figure 3.A.26 shows one of the pre-stack CMP seismic gathers input to the inversion. This figure indicates that the variation of seismic amplitude with offset is not significant compared to that shown in Figure 3.A.18 for a larger value of average porosity. The lack of measurable seismic amplitude variations with offset explains why the inversion rendered a porosity distribution that correlates poorly with the original distribution of porosity. 


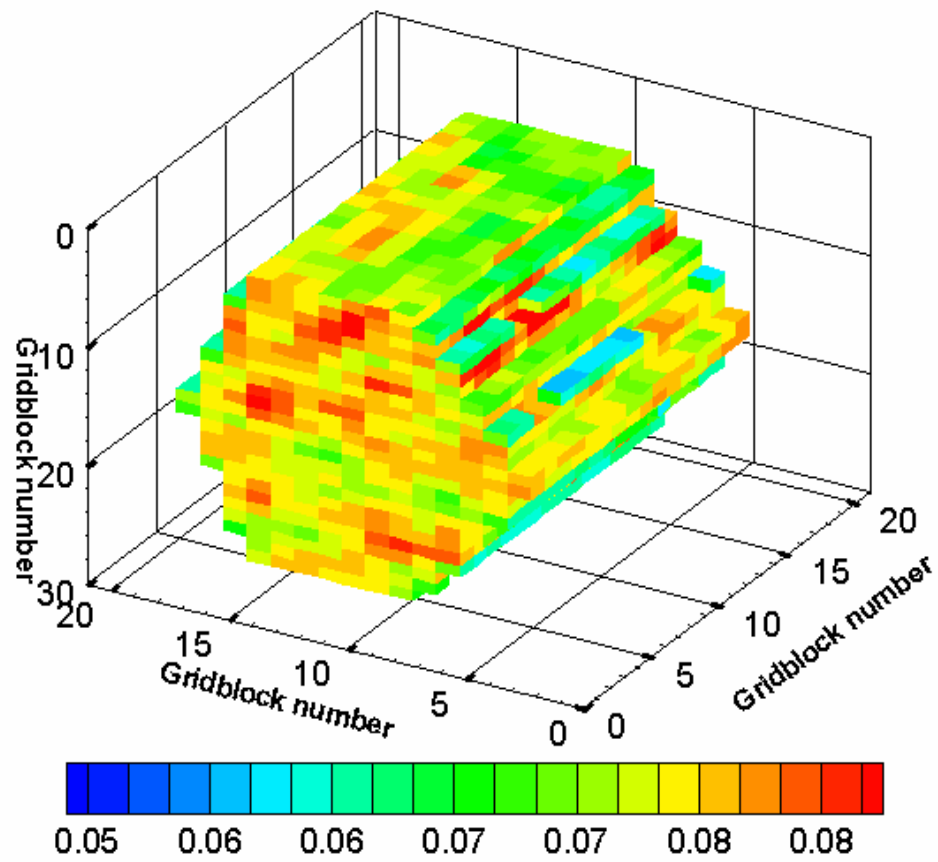

Figure 3.A.24. Graphic depiction of the spatial distribution of porosity for the case of a tight synthetic reservoir model.

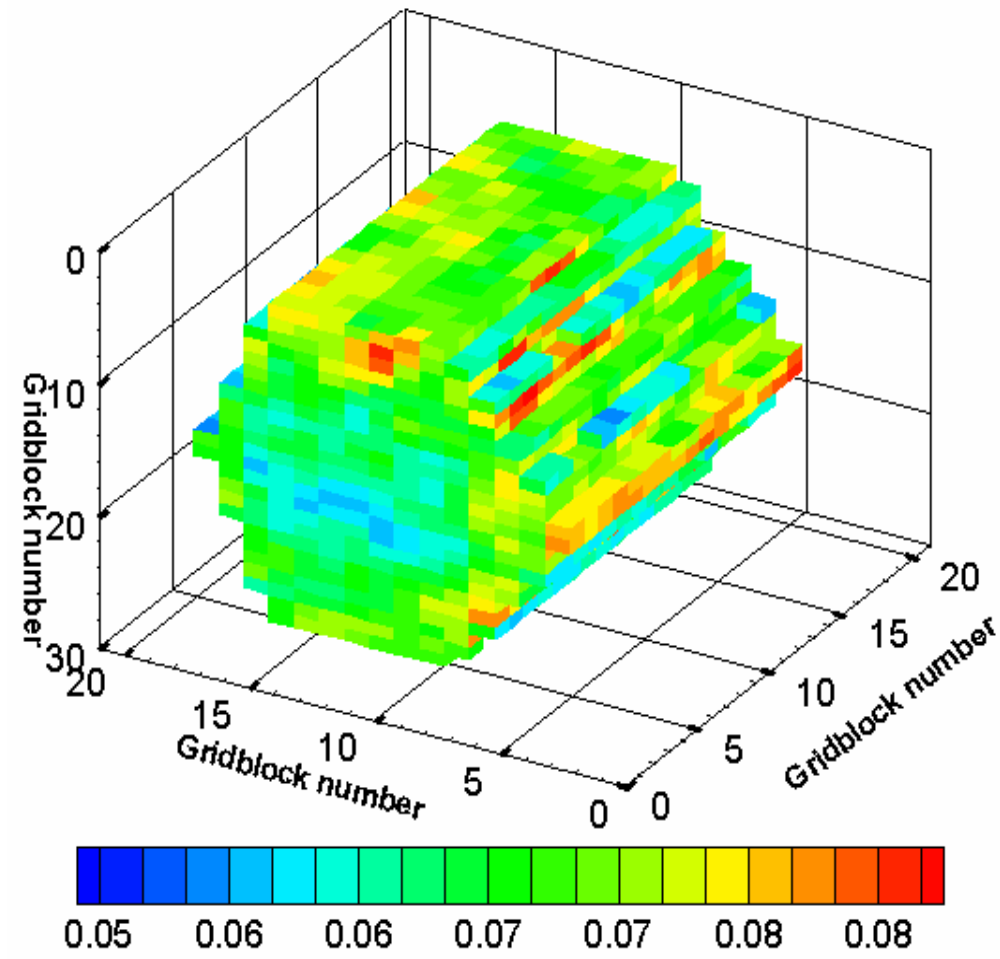

Figure 3.A.25. Graphic depiction of the spatial distribution of porosity inverted from prestack seismic data simulated for the case of the tight reservoir model shown in Figure 3.A.23. 


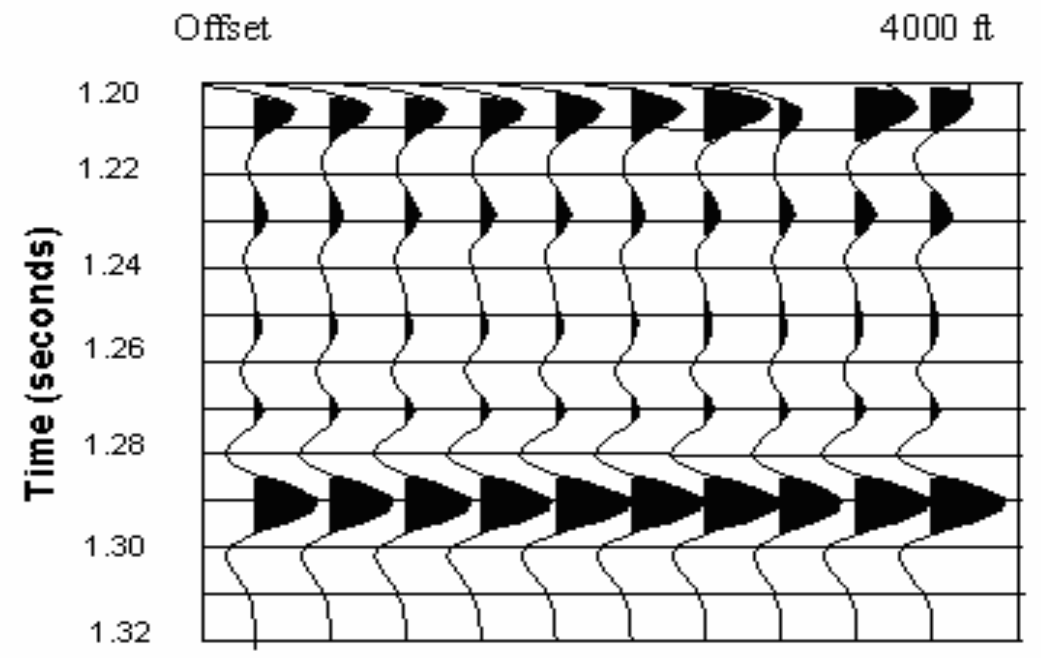

Figure 3.A.26. Simulated pre-stack CMP gather for the case of the tight reservoir model shown in Figure 23.

\section{A.2.5.5. Assessment of the Data Subspace Method}

In the preceding section we described the mathematical basis of the data subspace method. The choices of the sub-objective function and the calculation of the subspace vectors are the primary factors that control the efficiency of the proposed inversion method. A tradeoff must be made between CPU time and computer memory. If all of the observed data were grouped together, then the data sub-space method would turn into the steepest descent method, which entails low computer memory requirements at the expense of low rates of convergence. If each data point (or sample point) was selected to represent a single data sub-space vector, then the proposed data sub-space inversion would be equivalent to the Gauss-Newton minimization method. Too few or too many data sub-space vectors are not proper choices. In our studies, we found that the selection of data sub-space vectors by producer wells and by seismic offsets was an efficient choice. Figure 3.A.27 shows the convergence of the objective function as a function of iteration number for the two cases. 


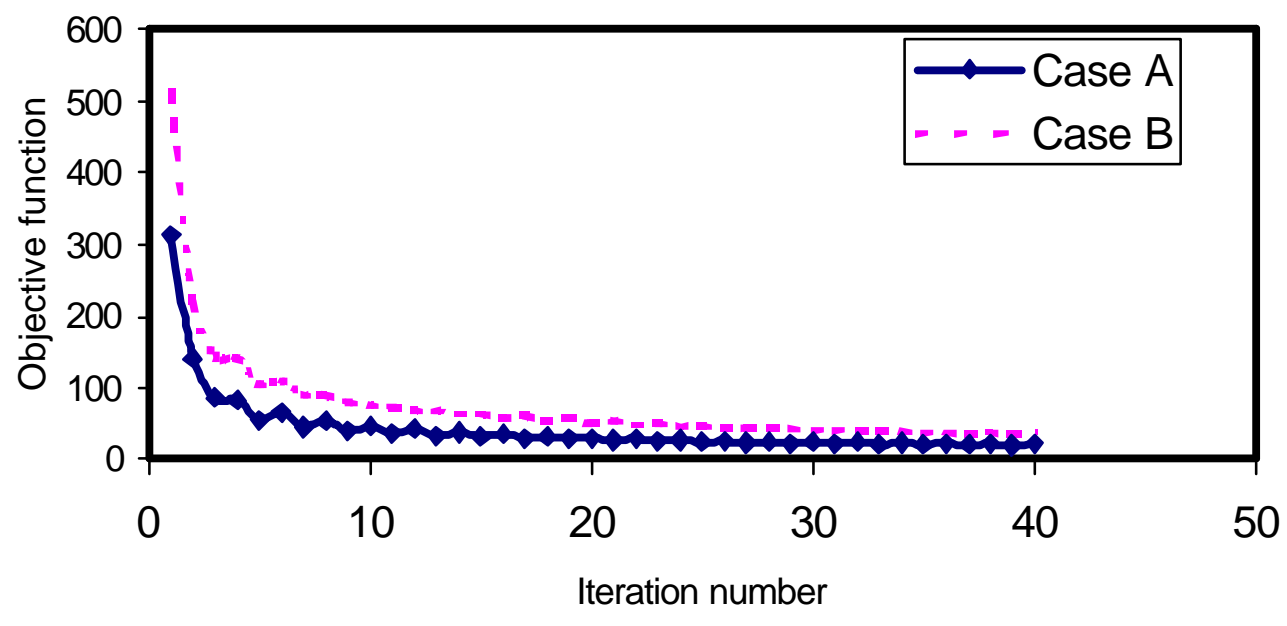

Figure 3.A.27. Evolution of the cost function used for inversion as a function of iteration number. The cost function is constructed using the data subspace algorithm described by equation (3.A.6). Case A and Case B correspond to different porosity models used to initialize the inversion.

It can be observed that, after a few iterations, the inversion converges to an optimal solution. Occasionally, variations in the initial guess model result in diverse inverted spatial distributions due to the non-uniqueness and instability of the inversion. However, for the inversion of pre-stack seismic data, we used different initial guess models but the inverted models remained very similar. Figure 3.A.28 shows the spatial distribution of porosity inverted from an initial spatially uniform porosity distribution chosen far from the mean of the original porosity distribution. We observe that the estimated porosity distribution is almost identical to that of the original case thereby indicating the stability of the inversion. 


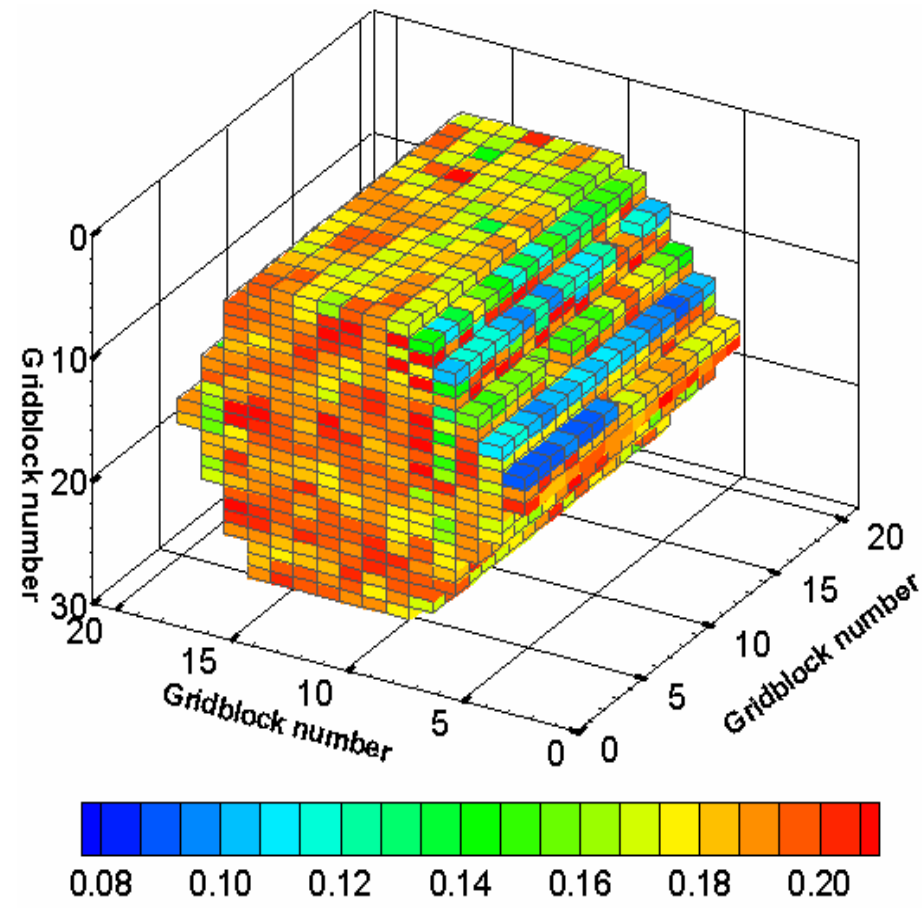

Figure 3.A.28. Graphical description of the spatial distribution of porosity inverted from prestack seismic data simulated for the tight reservoir model shown in Figure 3.A.23.

\section{A.2.5.6. Joint Inversion of Pre-stack Seismic Data and Time Records of Fluid}

\section{Production Measurements}

This test example is intended to assess the value of pre-stack seismic data on the estimation of permeability. From a practical viewpoint, the joint inversion is computationally challenging and CPU intensive. The proposed data sub-space inversion approach allows one to efficiently combine both pre-stack seismic data and fluid production measurements for the estimation of spatial distributions of porosity and permeability. As discussed in the previous cases, the porosity field can be stably and reliably computed from the inversion of pre-stack seismic data. In this case, we consider the joint inversion in two stages. After porosity estimates are obtained from the pre-stack seismic data invasion, those porosities are fixed and the second stage of the joint inversion focuses on the estimation of the spatial distribution of permeability only. Figure 3.A.29 shows the spatial distributions of 
permeability inverted with the use of pre-stack seismic data in addition to time records of fluid production measurements. Compared to the spatial distribution of permeability inverted from time records of fluid production measurements alone (shown in Figure 3.A.7) the use of pre-stack seismic data does improve the spatial resolution of the permeability field in the vicinity of wells. However, between wells the inverted spatial distribution of permeability is not satisfactory.

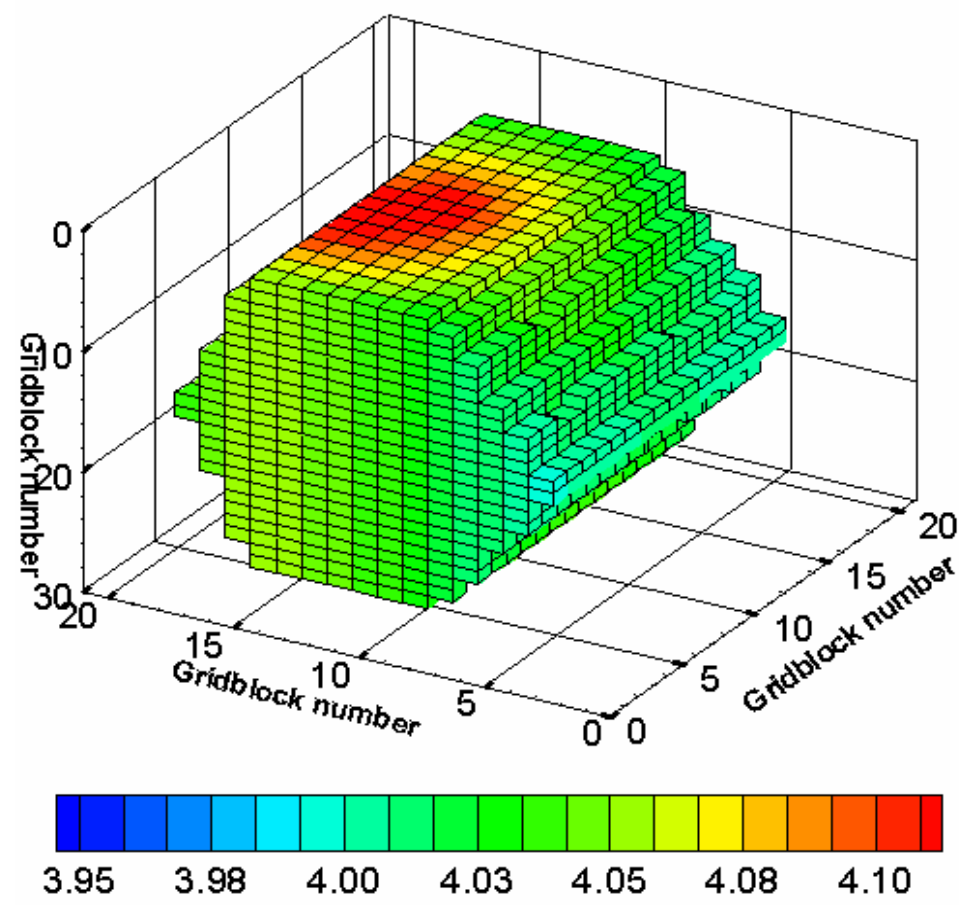

Figure 3.A.29. Graphic depiction of the spatial distribution of $\log$-permeability $[\log (\mathrm{md})]$ obtained from the joint inversion of pre -stack seismic data and time records of water-oil ratio. Data input to the inversion were generated from the tight reservoir model shown in Figure 3.A.23.

The above exercise indicates that pre-stack seismic data clearly provide much higher spatial resolution in the reconstruction of porosity than in the estimation of permeability. One reason for the poor spatial resolution for permeability even when pre-stack seismic data are combined with time records of fluid production measurements is that the WOR data are sensitive to large spatial averages of the permeability distribution in the vicinity of wells. However, the most important reason is that permeability is not directly related to seismic 
data. We believe that, in addition to WOR data, more information such as accurate correlation model between permeability and porosity or the availability of production measurements for each individual flow unit are necessary for further improving the spatial resolution of permeability. For example, recent studies have shown that the incorporation of in-situ permanent sensor measurements can dramatically enhance the spatial resolution of the inverted permeability (Wu et al., 2003).

\section{A.2.6. Discussion and Conclusions}

The inversion of time records of fluid production measurements into spatial distributions of total porosity and permeability is highly non-unique and unstable due to insufficient and inadequate data. Consequently, a large number of very diverse reservoir models can be constructed that will honor the measured production data within the assumed noise threshold. Non- uniqueness can only be overcome with the addition of independent pieces of data or else by reducing the parameter space through the enforcement of model constraints. The purpose of this paper was to explore the possibility of reducing nonuniqueness in the estimation of spatial distributions of porosity and permeability by combining time records of fluid production measurements with pre-stack 3D seismic data.

The synthetic examples of inversion described in this paper indicate that the use of pre-stack seismic data can significantly improve the resolution of reservoir parameters. Specifically, a good reconstruction of spatial distribution of porosity was obtained when the mean value of porosity was above ten percent. It was also found that the joint inversion of pre-stack seismic data and water-oil ratio data is still not sufficient to estimate a realistic spatial distribution of permeability. In order to improve the estimation of permeability it is necessary to establish a strong statistical correlation between permeability and porosity, or 
else to incorporate additional independent data such as permanent sensor and bottom-hole pressure measurements, for instance.

It must be pointed out that the success of the joint inversion approach described in this paper relies on the accuracy of the rock physics/fluid substitution model. The assumed correlation between elastic and reservoir parameters plays an important role in the inversion. For example, we assumed that the elastic bulk modulus and porosity were independent from the corresponding values of pressure and fluid saturation. If the influence of pressure and fluid saturation on the elastic bulk modulus were significant, then a more complex rock physics/fluid substitution model would be necessary to link elastic and petrophysical parameters.

The inversion of pre-stack seismic data provides a quantitative way to naturally bias the inversion toward a geologically consistent set of solutions in model space. Such an inversion strategy becomes appropriate when seismic amplitude data are related to the underlying reservoir parameters. 


\section{B. INSTITUTE FOR GEOPHYSICS}

\section{B.1. ABSTRACT}

During the third year funding period, the UTIG group conducted two main tasks:

(a) Rigorous evaluation of a parallel pre-stack waveform inversion code in the $\tau$-p domain: performance statistics on a PC cluster were evaluated and field data were inverted using the parallel code, and

(b) Direct estimation of petrophysical parameters from seismic data by pre-stack inversion: Together with CPGE we did theoretical development and numerical implementation which were tested with models generated from real well-logs. In addition we worked jointly with CPGE group in the development of joint inversion of seismic and fluid flow data.

One manuscript based on the parallel seismic inversion was submitted to a journal Concurrency and Computation, which has been accepted for publication. A paper on direct inversion of petrophysical parameters is currently under preparation. One paper on the theoretical development of a pre-stack waveform inversion was published in the Nov.-Dec. 2003 issue of Geophysics. One paper on the analysis of the Gulf of Thailand data has been accepted (subject to minor revision) in Geophysics. Copies of the manuscripts are attached in this report. In the following, we summarize some of the results.

\section{B.2. FULl WAVEFORM SEISMIC INVERSION USING A DISTRIBUTED S YSTEM OF COMPUTERS}

\section{B.2.1. Introduction}

The aim of seismic waveform inversion is to estimate elastic properties of earth's subsurface layers from recordings of seismic waveform data. This is usually accomplished by using constrained optimization often based on very simplistic assumptions. Full 
waveform inversion uses a more accurate wave propagation model but is extremely difficult to use for routine analysis and interpretation. This is because computational difficulties arise due to 1) strong nonlinearity of the inverse problem, 2) extreme ill-posedness and 3) large dimensions of data and model spaces. We show that some of these difficulties can be overcome by using (1) an improved forward problem solver and efficient technique to generate sensitivity matrix, (2) an iteration adaptive regularized truncated Gauss-Newton technique, (3) an efficient technique for matrix-matrix and matrix-vector multiplication and (4) a parallel programming implementation with a distributed system of processors. We use message-passing interface (MPI) in the parallel programming environment. The details of the optimization algorithm are outlined in Roy (2002), Sen and Roy (2003) and Roy et al. (2003). We will briefly summarize the algorithm below following which we will describe the parallelization aspect in detail.

\section{B.2.2. Algorithm of regularized Gauss-Newton}

The regularized Gauss-Newton as implemented here, differs from the ordinary GaussNewton by the way in which the Gauss-Newton update is selected. It is also shown in Roy (2002) that such an update ensures robust descent. We compute regularized Gauss-Newton update corresponding to each a posteriori regularization parameter $\alpha$ by minimizing Tikhonov's functional $T_{K}$, using nonlinear conjugate gradient (NLCG) method. We prefer NLCG because of its intrinsic property of providing stable and robust computational regime and minimum storage requirements. In the box below we describe our algorithm with a pseudo code. 
Let $\mathbf{m}_{0}$ be the starting model describing the distribution of elastic parameters in a layered model, at the outset of the Gauss-Newton optimization, $\tau$ is the pre-assigned threshold limit for the error functional $E_{k}$ (it measures the misfit between observed and synthetic data) to attain at its minimum and $\eta$ is the noise estimate in the data.

Set maximum iteration counter (ITMAX); $\mathbf{m}_{\text {new }}=\mathbf{m}_{0} ;$ compute $F\left(\mathbf{m}_{\text {new }}\right) ; \mathbf{G}$ and $E_{k}$

$$
\begin{aligned}
& \text { For } k=0,1,2, \ldots \\
& \text { If either } E_{k}>\tau \text { or } k<\text { ITMAX } \\
& \quad \text { Set } \mathbf{m}_{\text {old }}=\mathbf{m}_{\text {new }} \\
& \text { Minimize } \tilde{T}_{k} \text { (Eq. 14) through NLCG, which gives } \Delta \mathbf{m}^{\alpha} \\
& \text { Update } \mathbf{m}_{\text {new }}=\mathbf{m}_{\text {old }}+\Delta \mathbf{m}^{\alpha} \\
& \text { Else if } E_{k}>\tau \text { and } k \geq \text { ITMAX ; Retry with new starting model } \\
& \text { Compute a posteriori } \alpha \text { using Eqs. (17) and (18) } \\
& \text { Else "Print new solution upon convergence" } \\
& \text { End If } \\
& \text { End Loop }
\end{aligned}
$$

\section{B.2.3. Computational Issues}

While developing an inversion algorithm, the computational efficiency and robustness of the algorithm are of primary concern. Since in a model-based inversion scheme, the forward problem solver gets executed repeatedly, efficiency of forward computation plays a major role in computational efficiency of the algorithm. Our data and model spaces are very large; hence dimension of the sensitivity matrix is also very large. For example, if in an ensemble of seismic traces there are 40 seismic traces, each with 512 samples, the dimension of the data vector is 40x512 (20480). Again if the layered earth model consists of 512 layers then the dimension of the model vector is $3 \times 512$ (1536). We therefore immediately realize that major computational time in Gauss-Newton optimization will be consumed by matrix-matrix and matrix-vector multiplications. 


\section{B.2.3.1. Matrix multiplication-fast implementation}

Matrix-matrix multiplication is an essential computational step in realizing GaussNewton optimization. In a standard sequential algorithm the time complexity of multiplication of transpose of the sensitivity matrix $\mathbf{G}$ of order $(N \times M)$ with itself is $O\left(M N^{2}\right)$. If we assume that $\mathbf{G}$ is a square matrix of order $N$, then a sequential algorithm can achieve the best possible order of time complexity $O\left(N^{2.3755}\right)$. We, however, desire a more substantial reduction in complexity order, which is only possible if the matrix is considerably sparse. In our application, we identify that the $\mathbf{G}$ matrix is indeed sparse (Fig. 1). Note that a change in the parameters of one layer affects the response of all the layers below it. In other words, the shallowest layer affects contributions of all the layers and the deepest layer only affects the contribution by itself. Thus for each seismogram ( $p$ trace), we compute delay times for the layer in consideration and that of the deepest layer to compute a time window that is used to define a band in the $\mathbf{G}$ matrix for use in $\mathbf{G}^{T} \mathbf{G}$ evaluation. We also include the width of the source wavelet in defining the band. Note that the computation of the bandwidth is based on 'P-wave primaries only' model. A tolerance in it ( 25 samples) in either end works well in general. This reduces computation cost in sequential algorithm for matrix-matrix multiplication significantly (Fig. 2) resulting in an increase in computation speed by a factor of seven. However, on a distributed memory parallel computer (DMPC) architecture with $P$ processors, a fully scalable parallel algorithm for matrix-matrix multiplication would take $O\left(N^{\alpha} / P\right)$ time, where $N$ is the order of the matrices and $2<\alpha \leq 3$. 

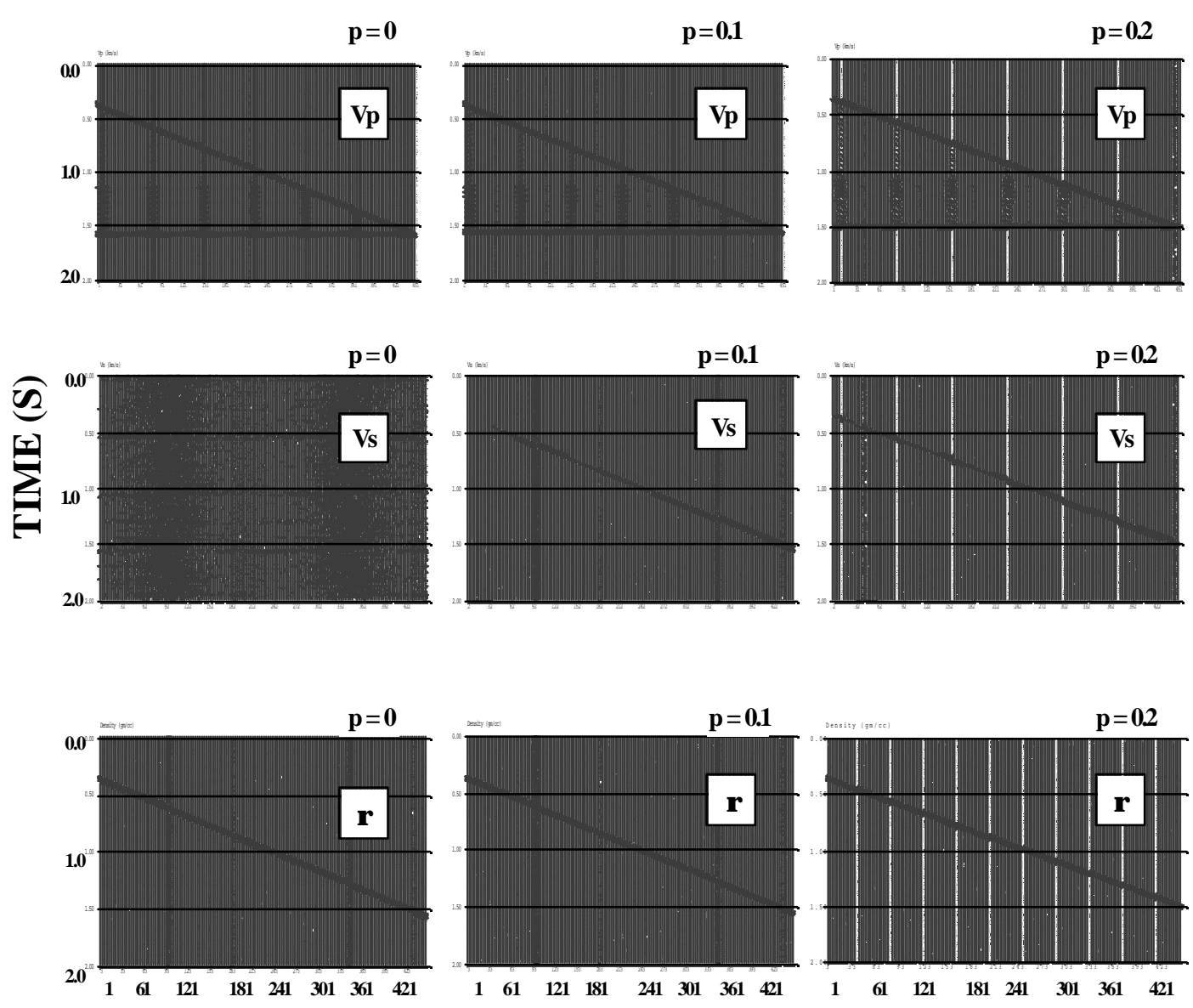

Number of Layers

Figure 3.B.1. Plots of differential seismograms with respect to P-wave velocity (top row), Swave velocity (middle row) and density (bottom row) of different layers of an earth model for three different values of ray-parameters $(0.0,0.1$ and $0.2 \mathrm{sec} / \mathrm{km}$ at the left, moiddle and right column respectively): Each differential seismogram is a vector of Frechet derivatives with respect to model parameters. It is obvious that for $\mathrm{p}=0$, the seismograms are not sensitive to changes in the shear wave velocity.

\section{B.2.3.2. Truncated Regularized Gauss-Newton}

We used a truncated regularized Gauss-Newton (TRGN), a variant of RGN, to improve the computation efficiency of the inversion algorithm. At the onset of RGN while the starting model is far from the optimal one, a precise estimate of RGN update does not make much difference in the updated model (Nash, 2000). On the other hand, a precise estimate of the RGN update will be necessary as the updated model approaches the optimal 
one. Thus, the wasteful computation for a precise estimate of the model update can be avoided if any early termination in the inner loop of RGN is invoked adaptively. Dembo and Steihaug (1983) originally proposed such strategy of early termination in a large-scale unconstrained optimization problem through a truncated Newton (TN) algorithm. However, invoking early termination not only lowers the computational burden but also offers an additional regularization in the computation.

\section{B.2.3.3. Parallel computation}

Easy availability of low cost high performance computational facilities, demand of intrinsically high computational cost due to the presence of strong nonlinearity in a full waveform pre-stack inversion and large dimension of the computational regime (large data and model spaces) are some of the motivating factors for parallelization of the inversion algorithm. As our interest lies with resource utilization and portability of the software in different platforms, we design our algorithm using message-passing interface (MPI) on distributed memory parallel computer (DMPC) with a homogeneous cluster of PCs that are connected with a high speed network. A DMPC consists of finite number of processors each with its own local memory. These processors (if identical) form a homogeneous cluster and communicate with each other via MPI using high-speed network. The computations and communications in DMPC are globally synchronized into either computation or communication step. In any of the steps, a processor either remains in operation or stays idle. Hence, a busy processor in a computation step generally performs arithmetic or logical operation and elapses a constant amount of time. On the other hand, in a communication step, the processors send and receive messages via network and build a one-to-one communication. Note that each processor can receive at most one message in a communication step and 
elapse time of communication for each processor is assumed to be constant. Hence, the time complexity of a parallel computation on a DMPC is the sum of number of computation steps and communication steps. To reduce the wait time in communication mode, the implementation of fast electronic network is important. In our present numerical experiment, we use 32 PCs aided with AMD Athlon processors, 1GB RAM connected in a Linux cluster interconnected with high speed Myrinet ${ }^{\mathrm{TM}}$.

\section{B.2.4. Algorithmic structure}

For a nonlinear optimization problem, Schnabel (1995) identified three stages of parallelization; they include (1) parallelization of function and/or the derivative evaluation in the algorithm; (2) parallelization of linear algebra kernels; and (3) modifications of the basic algorithms, which increase the degree of intrinsic parallelism. While a coarse-grained parat lelism can be invoked to the stages (1) and (3) of parallelization, fine-grained parallelism often becomes necessary in stage (2) of parallelization.

In many occasions, with a single level parallelism, a coarse-grained parallelism is favored over fine-grained parallelism. The reasons for such preference are (1) availability of concurrency at high-level language representation, and (2) superiority of concurrency achieved over fine-grained parallelism in a distributed system. Note that in a fine-grained parallelism, concurrency is available at low-level language representation. In our parallel implementation of the inversion algorithm, we have primarily adopted a single level coarsegrained parallelism. Within the DMPC architecture while evaluating a numerical method, a coarse-grained parallelization requires very little inter-processor communication and therefore loss of parallel efficiency due to communication delay as the number of processor increases (assuming that there are enough separable computations to utilize the additional 
processors) is insignificant. Fine-grained parallelism, on the other hand, involves much more communication among processors and care must be taken to avoid the case of nefficient machine utilization in which the communication demand among processors outperform the amount of actual computational work to be performed. We will discard fine-grained paral lelization in the present context as we primarily focus on single level coarse-grained parallelization of the algorithm. We adopt here a master-slave paradigm using the MPI standard (Snir et al. 1996). Such paradigm increases granularity as desired. Figure 2 is the schematic plot of the algorithmic architecture that we have implemented in our application.

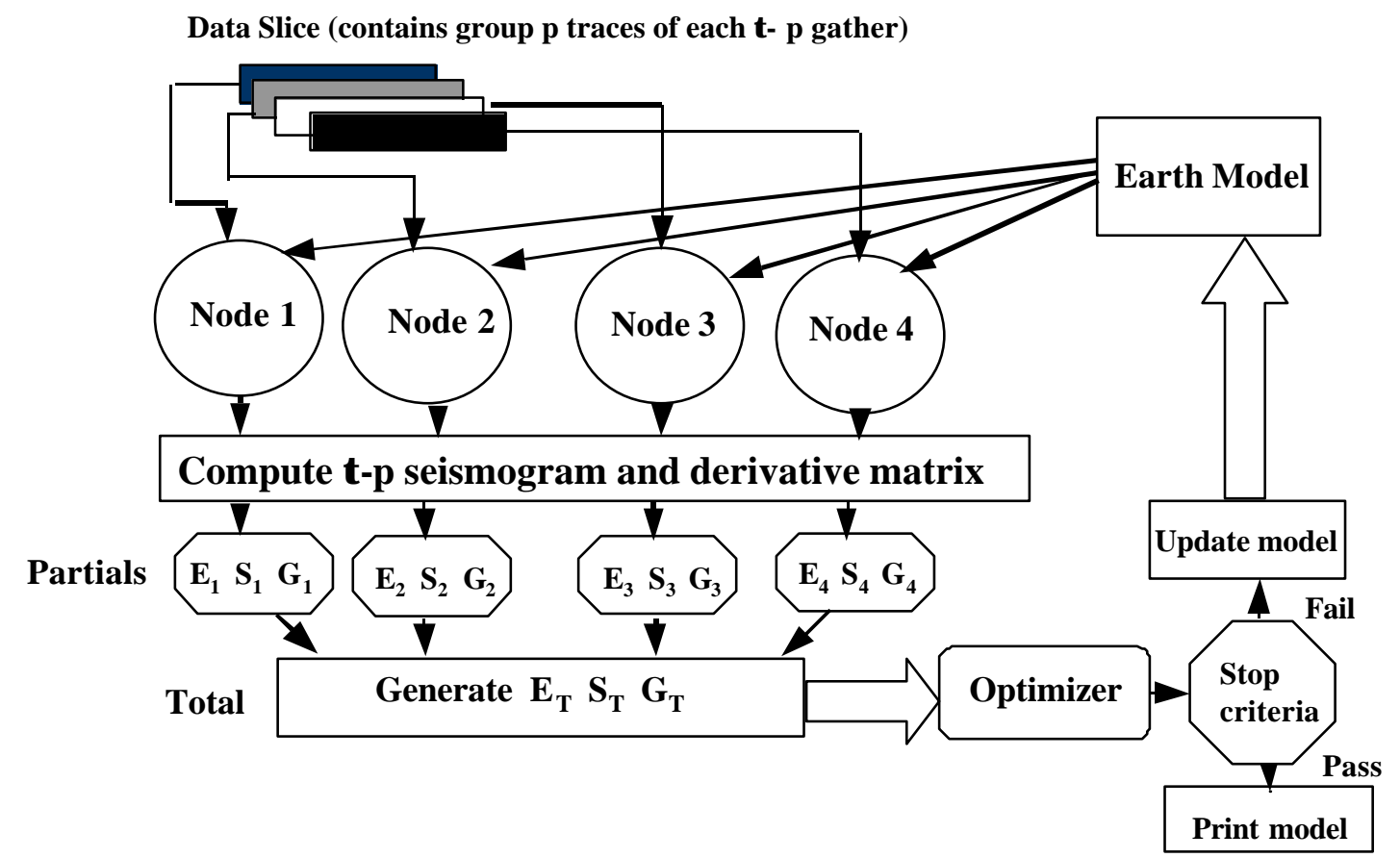

Figure 3.B.2. Schematic plot of algorithmic structure. The data are distributed from master node to several nodes (slave). Note that algorithm uses single program multiple data (XPMD) type parallel computation. $\mathrm{F}_{i} \mathrm{~S}_{i}, \mathrm{G}_{i}$ correspond to the partial values of error function, sensitivity matrix and $\mathrm{G}^{T} \mathrm{G}$ computed at processor $i$. The totals $\mathrm{E}_{i}, \mathrm{~S}_{i}, \mathrm{G}_{i}$ are computed using a global 2 sum.

Depending on the numerical method being designed, the first step of parallel algorithm is the module specification in which a method is decomposed into appropriate sub-methods with 
necessary specification of data dependency of entire data structure. All such sub-methods are recognized as modules. Once, the module is specified, the next step is to design schedule for the execution of each independent module. As in DMPC architecture, the communication between each processor is one-to-one and is of constant time, the design of the schedule becomes rather simple. The remaining last step is load balancing at each node, which is primarily a data distribution strategy.
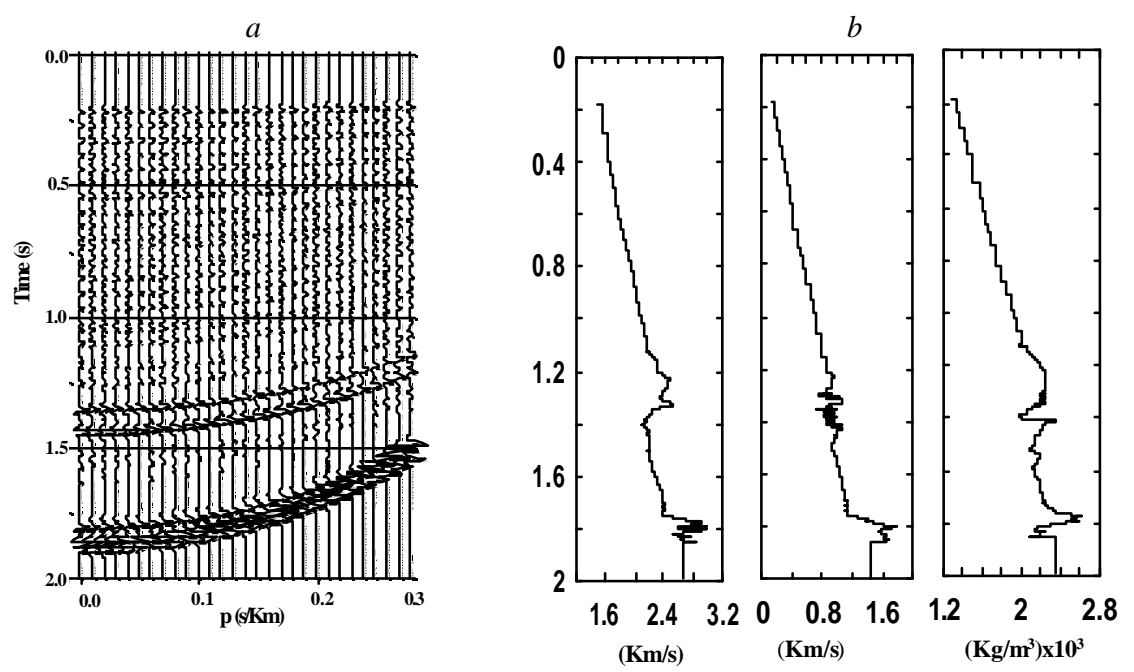

Figure 3.B.3. (a) Plot of synthetically generated t-p seismograms with 5\% random noise derived from a true earth Model presented in the adjacent panel. The source wavelet used is Ricker wavelet with $35 \mathrm{~Hz}$ peak frequency. (b) Plot of P-, S-velocities and density with twoway time. Note that the layer thickness is expressed in terms of two-way travel time by Pwave in a medium.

\section{B.2.4.1. Load balancing strategy}

Load balancing is the method of dividing the amount of work to two or more processors so that an optimal amount of work gets done in the same amount of time with a minimal wait-time for each processor. Load balancing can be implemented with hardware, software, or a combination of both. A successful load balancing improves the scalability of the parallel program. A pure exploitation of parallelism does not lead to scalable parallel programs if the number of concurrent modules is not equal to the number of processors. 
Note that in our application (Figure 3.B.3) of inversion of plane wave seismograms, computations of plane wave seismograms are independent of each other.

Thus we distribute the tasks of generation of plane wave seismograms and their sensitivity matrices to different processors. If there are $N$ seismograms to be modeled and the number of available processors is $P$, each processor is assigned $N / P$ seismograms. If $N$ is an integral multiple of $P$, we have perfectly balanced distribution of tasks. Otherwise, the tasks are so distributed that difference in load distribution between the different processors is minimal. Note that with a single program multiple data (SPMD) programming paradigm, such a strategy has the advantage of providing high scalability. Once the synthetic seismograms and the sensitivity matrices are computed, they are transferred to the master node where model updates are computed using the Newton update formula.

\section{B.2.5. Performance analysis of algorithm}

We have implemented the parallelized version of the full waveform inversion algorithm for 2-D seismic data in pre-stack domain. The 2-D seismic data are represented by groups of seismic traces, where each group corresponds to a surface nodal point (also called common mid-point or CMP gather) of the 2-D grid. In order to realize inversion of entire 2D seismic data (also known as 2-D seismic line), we invert seismic data corresponding to each surface nodal point. Thus, our parallelized version of inversion algorithm in any computation cycle handles groups of seismic traces belonging to a CMP. Note that we have used $(\tau-p)$ transformed seismic data in our algorithm. We invoked master-slave paradigm where in master node we first divide the group (domain) into subgroups (sub domain) such that size of the sub groups remains the same, i.e., in each sub group we assign certain number of seismic traces. Efforts are made to distribute the traces equally, if possible. Otherwise the 
differences are kept to a minimum. Each sub group is then allocated with the processing elements (PE) as slaves. Each PE reads the earth model and computes synthetic response and the sensitivity matrix corresponding to the designated observed seismic traces. Once computation is completed, all results are summed in the designated node (usually master node), where optimization code runs. Efficient summation is achieved using a power of 2 global sum method (Sen et al., 1999). We use our algorithm on both the test case and real data set. The run of the algorithm on the test case is important, as it allows the study of the applicability of the method to real world situation and performance analysis of the algorithm. In our performance analysis test for parallel algorithm, the dimension of both data and model spaces play a major role in computational demand. Hence, we will focus mainly how parallel algorithm affects on data and model space dimensions and the number of PE used in the computation. In our test case, we use two sets of $(\tau-p)$ seismic gather with 30 and 60 traced within a ray-parameter range of $0-0.3 \mathrm{sec} / \mathrm{km}$. Each trace contains 512 data samples with a sampling interval of $4 \mathrm{~ms}$. The earth model is made up of three profiles corresponding to P- and S-velocity and density of the medium. The number of data points and the sampling interval of those profiles are 453 and $4 \mathrm{~ms}$ respectively. This means that the earth model is assumed to be a pack of 453 layers whose thickness in terms of two-way time is $4 \mathrm{~ms}$. Note that the two-way normal reflection time is computed by dividing the thickness with half the P-wave velocity of the layer. The synthetic data used in the performance analysis are shown in Fig. 3.B.5(a); the earth model parameters used in the computation of test seismograms are shown in Fig 3.B.5(b). We use the compute time for single iteration on the synthetic data set for a detailed performance analysis described below. 


\section{B.2.5.1. Elapsed time vs. number of processors}

In Figures 3.A.4(a) and 3.A.4(b) we plot elapsed time vs. number of processors and inverse of the number of processors respectively using 30 and 60 traces. Figure 3B.4(a) clearly demonstrates that with the increase of processors in the system elapsed time continues to fall. However, the rate of decrease slows down for the increase of processors from 10 to 20 in both the synthetic data examples. Figure 3.B.4(b) also depicts almost linear trend, which immediately indicates that the sequential components of the algorithm remains nearly constant with the processors.

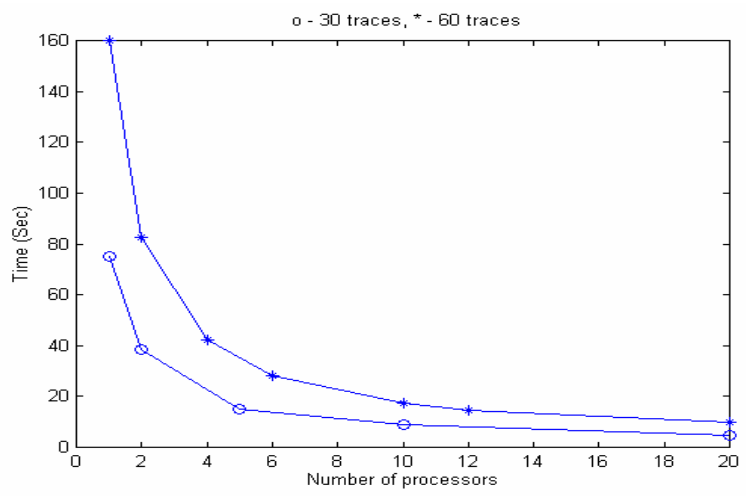

Figure 3.B.4. (a) The plot of the elapsed time versus the number of processors. Elapsed time decreases with the addition of the processor.

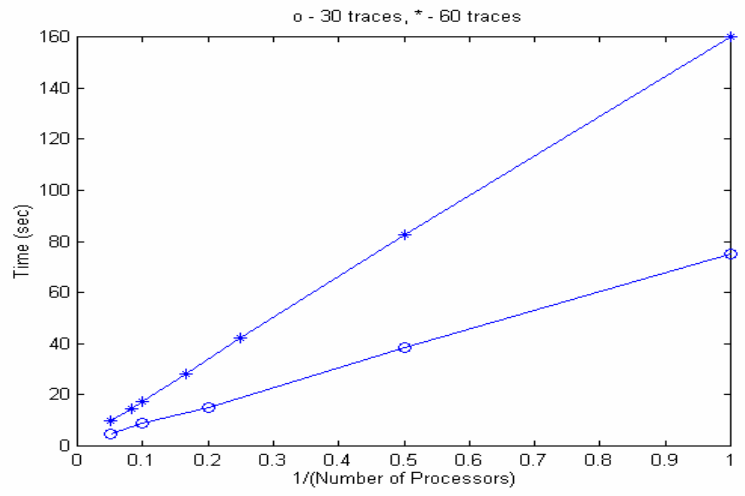

Figure 3.B.4. (b) Plot of the elapsed time versus the inverse of the number of processors: the plot is almost linear, indicating that the sequential components of the algorithm are nearly constant. Similar trends are observed for the two datasets containing 30 and 60 traces. 


\section{B.2.5.2. Speedup, overhead, efficiency, performance measure and efficacy on homogeneous clusters}

The speedup is the measure of acceleration for a parallel algorithm running on distributed processors with respect to the best sequential algorithm running on a sequential computer. Hence, with a parallel architecture if $T(P)$ is the time taken by $P$ processors in executing a parallel algorithm and $T(1)$ is the time taken by best sequential algorithm on a single processor then the speedup due to $P$ processors can be written as

$$
S(P)=\frac{T(1)}{T(P)} .
$$

In an alternative definition due to Amdahl (1987), if a parallel algorithm A is such that part of it, say $\alpha$ fraction (known as Amdahl fraction), is not parallelizable, then the speedup $S(P)$ is given by

$$
S(P)=\frac{P}{1+(P-1) \alpha} .
$$

The above equation suggests that the speedup can attain the maximum value $1 / \alpha$ no matter how many processors are used for parallel computation. Hence, if 5\% of the algorithm is not parallelizable, then the maximum possible speedup is 20 . In a most ideal situation, when $\alpha \approx 0$, the curve of speedup versus number of processors follows a linear trend else it deviates to a sub-linear trend and may saturate normally. The above definition of speedup thus provides the basis for selecting an optimal number of processors for parallel algorithm. Interestingly, with a given problem size, the efficiency (which is a measure of average fraction of time that each processor effectively use while running a parallel algorithm) decreases with the number of processors. Note that as the number of processors increases 
inter-processor communication cost, idle time due to synchronization etc increase; this is expressed in terms of a metric called overhead which is defined as

$$
O v=P T(P)-T(1)
$$

Therefore, there exists a functional relationship between efficiency and overhead and is expressed as

$$
E(P)=\frac{T(1)}{P T(P)}=\frac{1}{1+\frac{O v}{T(1)}}
$$

Thus, while overhead increases the efficiency of the parallel architecture decreases. However, substantial reduction of overhead can be achieved by increasing the granularity, which is measure of amount of computational work done before processors have to communicate. Figures. 3.B.5(a), 3.B5(b), and 3.B.5(c) are the plots of speedup, overhead and efficiency versus number of processors for the two sets of synthetic data. The speedup curve demonstrates a near linear trend, which indicates a good scalability of the algorithm and suggests that only a small fraction of code used is not parallelizable. We have found that only about $2 \%$ of our code is not parallelizable. A full-scale parallelization of an algorithm for a highly nonlinear inverse problem is difficult to achieve unless the algorithm is intrinsically decomposable. Nevertheless, in most practical situations full scale decomposition and parallelization of an algorithm is not advisable as overhead grows considerably with the addition of the processor. We observe (Fig. 3.B.5(b)) that the overhead is minimal using five processors and it increases with the increase of number of processor. However, if the problem size increases on a fixed number of processors, efficiency increases. We may keep the efficiency fixed and increase the problem size and number of processors, as 
overhead increases slower than the problem size. This indicates good scalability of our algorithm.

We have applied the inversion algorithm to both synthetic and field seismic data. Inversion results for the synthetic example shown in Figure 3.B.3 are presented in Figures 3.B.6(a) and 3.B.6(b). Note that data-fit is excellent; so is the model recovery. Convergence

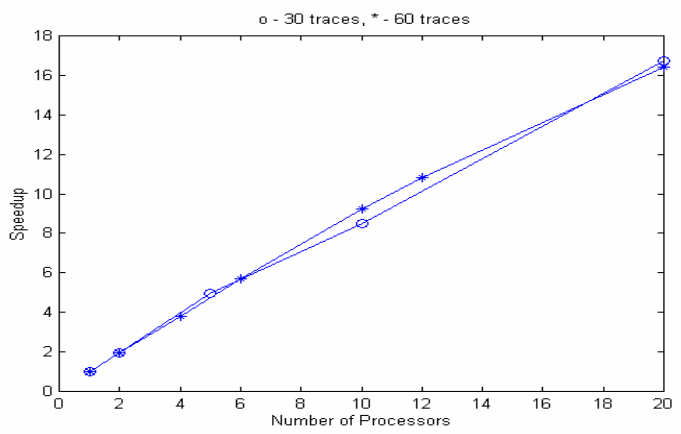

(a)

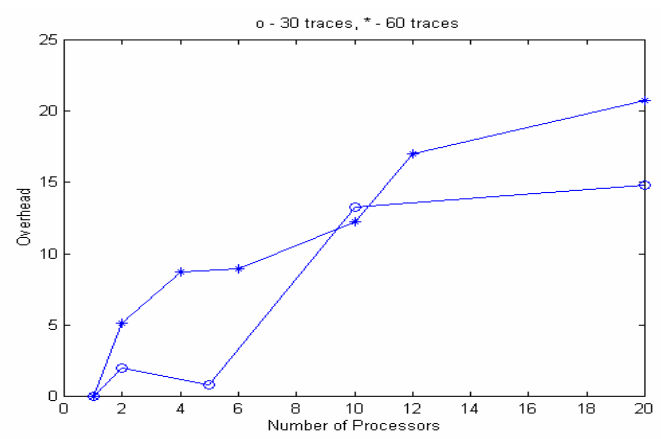

(b)

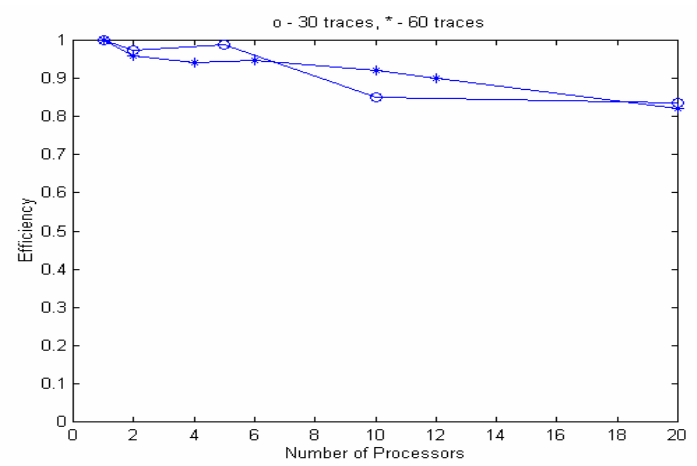

(c)

Figure 3.B.5. (a) Plot of speedup versus the number of processors: speedup curve nearly follows a linear trend indicating good scalability. (b) Plot of overhead versus the number of processors, and (c) plot of efficiency versus the number of processors. Similar trends are observed for the two datasets. 
was reached in 50 iterations. The results obtained are in good agreement with the true model.

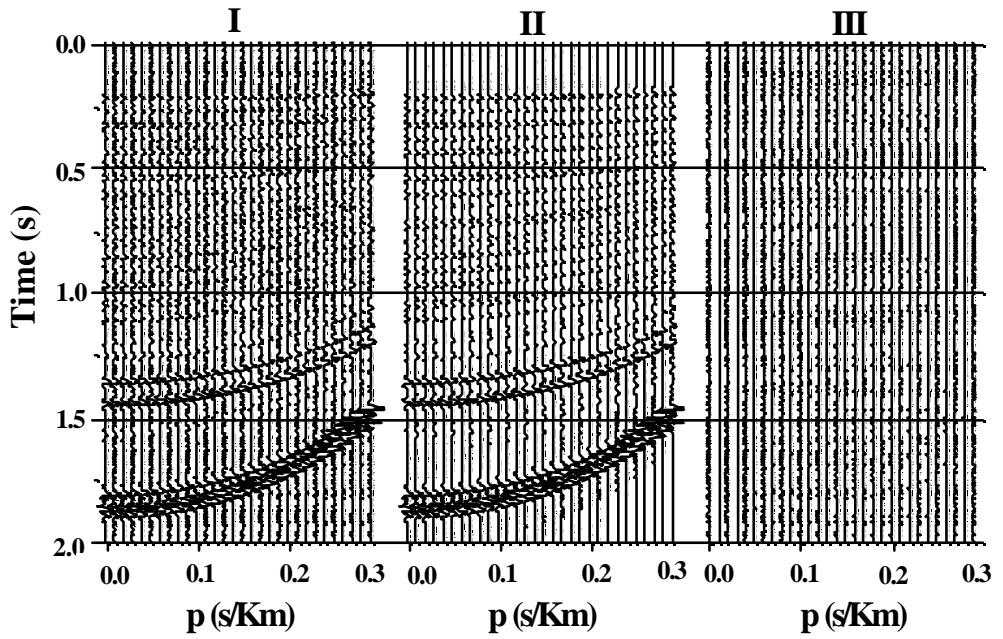

(a)
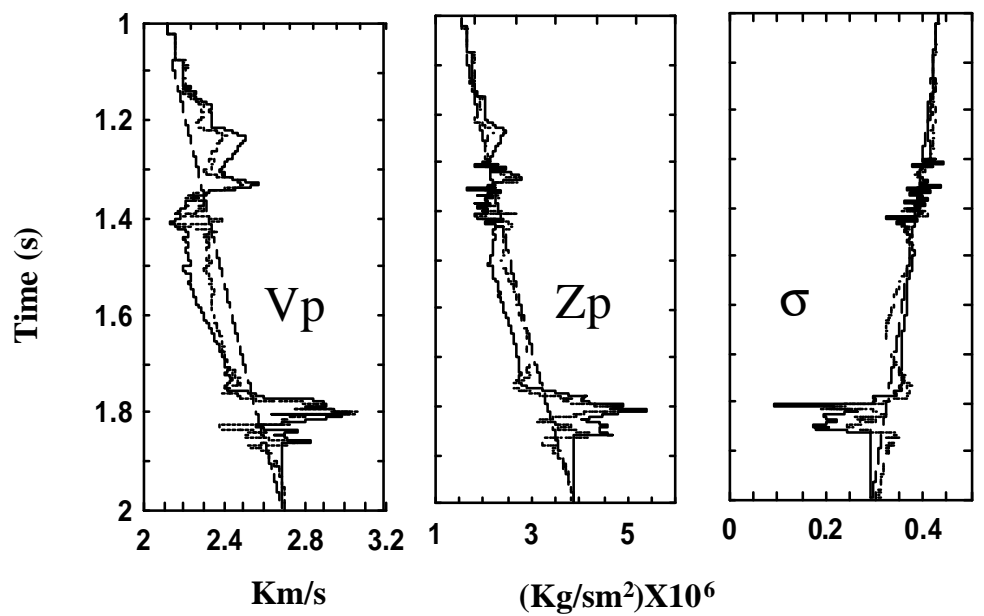

(b)

Figure 3.B.6. (a) Plot of data fir presented in three panels; the left panel is observed data, the middle panel id the best fit data and the right panel is the data residual. (b) Plots of true (solid line), initial guess (dashed line), and the inverted model (dotted line) 


\section{B.3. DIRECT ESTIMATION OF PETROPHYSICAL PARAMETERS VIA FULL WAVEFORM INVERSION OF PRE-STACK SEISMIC DATA}

\section{B.3.1. Introduction}

A detailed study of static and dynamic behavior of a producing hydrocarbon reservoir is essential for the production planning of a reservoir. Interestingly, the characterization of a reservoir can primarily be realized through an estimate of spatio-temporal variability of petrophysical properties, such as porosity, fluid saturation, permeability, pore pressure etc., in the precinct of a given geological model for the reservoir. With the aid of modern wireline measurements augmented by core analysis, it is possible to estimate the one- dimensional variability of the petrophysical parameter in the close vicinity of a well fairly precisely. However, the approach is insufficient to account for lateral heterogeneity of the petrophysical properties of the reservoir. It is widely acknowledged that it is impossible to delineate a subsurface map of both vertical and lateral variation of petrophysical parameters using a limited number of wells and well logs unless supplemented by some other methods, such as geostatistical estimates, inversion of seismic data, etc. Geostatistical methods used to estimate lateral heterogeneity of petrophysical parameters of a reservoir could be useful if the number of wells with necessary well logs is considerably large. On the other hand, a surface seismic method provides more detail lateral coverage, although vertical resolution is limited to the seismic scale. Thus, reflection seismic data can be even more useful if estimates of the petrophysical parameters can be made directly from seismic data.

The effect of variation of the petrophysical properties such as porosity, fluid saturation, and lithology on the elastic moduli of the rocks and hence on the seismic velocity field has been a much studied subject. However, little has been reported so far on the estimation of petrophysical properties of the medium directly from measured seismic wave 
field. The objective of this paper is to study the feasibility of determining petrophysical parameters directly from full waveform inversion of pre-stack seismic data. At the outset, we give a brief background of theoretical studies in the determination of relationship between petrophysical and the elastic parameters of the rocks.

\section{B.3.2. Relation between rock physics and petrophysical properties}

The seminal works in establishing a relationship between elastic moduli and petrophysical parameters are due to Biot $(1941,1956)$ and Gassmann $(1951)$ while studying the propagation of seismic waves through a pack of elastic spheres saturated with fluid. BiotGassmann theory allows the estimation of the bulk and shear moduli of a fluid-saturated rock formation via the equation

$$
K^{*}=K_{d}+\frac{K_{f}\left(1-\frac{K_{d}}{K_{m}}\right)^{2}}{\phi+\frac{K_{f}}{K_{m}}\left(1-\phi-\frac{K_{d}}{K_{m}}\right)},
$$

where, $\phi$ is total porosity and $K^{*}, K_{f}, K_{m}, K_{d}$ are the bulk modulii of the rock saturated with fluid, the fluid, the mineral grains and the rock skeleton or the frame, respectively, and

$$
G^{*}=G_{d}
$$

where, $G_{d}$ is the shear modulus of the frame.

It is assumed that fluid saturation plays an insignificant role in the variation of the shear modulus. Note that the frame moduli $K_{d}$ and $G_{d}$ are not the moduli due to dry rock; instead those are moduli of irreducible water-saturated rock. The above equation is based on the assumptions that the frequency of the seismic wave is low enough (theoretically zero) so that the studied rock can be considered macroscopically homogeneous, porosity and permeability are high enough, rock-fluid system is closed, and that fluids present in the rock 
should not interact with the rock matrix and must have negligible viscosity. The conditions described above are too stringent and hence are rarely met in most practical situations. The above equations work satisfactorily for unconsolidated water saturated sands (Wang, 2000). Thus, if all five input parameters are known then elastic moduli of a rock saturated with a fluid or a mixture of fluids can be obtained. However, precise estimates of all these input variables are difficult to obtain, as strong variability exists due to lithology, clay content, pore structure, pore aspect ratio, type of fluid content, etc. These input parameters are either measured in the laboratory or in situ using well logs. One of the most common log-derived parameters is porosity using either nuclear or acoustic logs. The bulk modulus of the fluid mixture can be calculated using Wood's equation (Wood, 1941) as

$$
\frac{1}{K_{f}}=\frac{S_{w}}{K_{w}}+\frac{S_{o}}{K_{o}}+\frac{S_{g}}{K_{g}},
$$

where, $K_{w}, K_{o}$ and $K_{g}$ are bulk moduli of water, oil and gas, respectively, $S_{w}, S_{o}$ and $S_{g}$ are the water, oil and gas saturations respectively. For any two phase fluid

$$
\frac{1}{K_{f}}=\frac{S_{w}}{K_{w}}+\frac{1-S_{w}}{K_{h c}} .
$$

Equation (4) can be simplified to

$$
K_{f}=\frac{K_{w} K_{h c}}{K_{h c} S_{w}+K_{w}\left(1-S_{w}\right)}
$$

If the laboratory measured data for bulk moduli of the individual fluid are available and if the water saturation of the rock is obtained from well logs and the well-established empirical formula, then bulk modulus of the rock saturated with a mixture of fluids such as water-gas or water-oil can be obtained. Note that there is a strong dependency of $K_{f}$ on the estimation of the water saturation. Mineral constituents of a rock also have a strong effect on 
the rock's elastic moduli. Elastic moduli of mineral grains $K_{m}$ and $G_{m}$ can be obtained using the Voigt-Reuss-Hill (VRH) average method (Hamilton, 1971) if the rock type and its mineral contents are known. If $M$ is the effective grain modulus which can be either $K_{m}$ or $G_{m}$ then $M$ can be written in terms of Hill average (Hill, 1952) as

$$
M=0.5 *\left(M_{v}+M_{r}\right)
$$

where $M_{v}, M_{r}$ are the Voigt (1928) and Reuss (1929) averages, respectively, given by

$$
M_{v}=\sum_{i=1}^{n} c_{i} M_{i}
$$

and

$$
M_{r}=\sum_{i=1}^{n} \frac{c_{i}}{M_{i}}
$$

where, $c_{i}$ and $M_{i}$ are the volume fraction and modulus of the $i$-th component, respectively. Note that although precise measurements of large number of mineral species are available, an accurate estimate of the volume fraction of the mineral grains present in the rock is not always possible especially from log measurements. For example, in a clastic sediment deposit, if the rocks are made up of only two species of mineral grains such as pure sand and clay (only with one variety of clay such as illite or kaolinite) then effective elastic grain moduli of the rock formation can be obtained using the above equations provided the volume fraction for each mineral present in the rock is known. It is possible to make an estimate of shale volume from natural gamma ray logs. However, $\mathrm{Xu}$ and White (1995) showed that VRH estimate is insufficient for sand clay mixture as it does not account for the aspect ratio of the pore space associated with clay and sand respectively. Moreover, log analysis of natural gamma ray logs gives shale volume not the clay content. $\mathrm{Xu}$ and White (1995) 
suggested the following time average equations to determine $\mathrm{P}$ - and S- wave velocities $V_{m}^{P}$ and $V_{m}^{S}$ of the mineral for a rock composed of a shale-sand mixture

$$
\frac{1}{V_{m}^{P}}=\frac{1-v_{s h}^{*}}{V_{s}^{P}}+\frac{v_{s h}^{*}}{V_{s h}^{P}},
$$

and

$$
\frac{1}{V_{m}^{S}}=\frac{1-v_{s h}^{*}}{V_{s}^{S}}+\frac{v_{s h}^{*}}{V_{s h}^{S}},
$$

where,

$$
v_{s h}^{*}=\frac{v_{s h}}{1-\phi},
$$

$\phi$ is the effective porosity estimated from $\operatorname{logs}$ and $v_{s h}$ is the percent volume of the shale in the rock. The bulk density of the rock can also be written as

$$
\rho_{m}=\left(1-V_{s h}^{*}\right) \rho_{s}+V_{s h}^{*} \rho_{s h} \quad \text {. }
$$

According to Gassmann (1951), the bulk modulus of the frame is related to the porosity of the rock through Biot's coefficient, $\beta$ (Biot, 1941), which is defined as the ratio of change in fluid volume to the change in formation volume. The relation is given by

$$
K_{d}=K_{m}(1-\beta)
$$

Lee (2002) proposed the following empirical relation to compute Biot's coefficient and showed that $\beta$ asymptotically approaches the value of one with an increase in the rock's porosity:

$$
\beta=\frac{\left(A_{1}-A_{2}\right)}{1+\exp \left[\left(\phi+\tau_{1}\right) / \tau_{2}\right]}+A_{2},
$$

where, $A_{1}=-183.05186, A_{2}=0.99494, \tau_{1}=0.56468$ and $\tau_{2}=0.10817$. 
Using equations 3.B.1 and 3.B.12 we obtain

$$
K^{*}=K_{m}(1-\beta)+\frac{K_{f} \beta}{\phi+\frac{K_{f}}{K_{m}}(\beta-\phi)} .
$$

Equation 3.B.14 can be rewritten as

$$
K^{*}=K_{m}(1-\beta)+\beta^{2} \xi \quad,
$$

where, $\xi$ is a modulus that measures the variation in hydraulic pressure needed to force an amount water into the formation without any change in the formation volume and is related to the Biot's coefficient $\beta$ through the equation

$$
\frac{1}{\xi}=\frac{(\beta-\phi)}{K_{m}}+\frac{\phi}{K_{f}}
$$

Therefore, the porosity $\phi$ and the water saturation $S_{w}$ play the most important role in determining the bulk modulus of a fluid-saturated rock. Contrary to Gassmann's (1951) assumption, Lee (2002) showed that the shear modulus also exhibits a dependence on the rock's water saturation, $S_{w}$, and proposed the following formula for the shear modulus of a fluid-saturated rock:

$$
G^{*}=\frac{G_{m} K_{m}(1-\beta)(1-\phi)^{2}+G_{m} \beta^{2} \xi(1-\phi)^{2}}{K_{m}+4 G_{m}\left[1-(1-\phi)^{2}\right] / 3} .
$$

The Effective formation bulk density for the fluid saturated rock can thus be written as

$$
\rho^{*}=(1-\phi) \rho_{m}+\phi \rho_{f},
$$

where,

$$
\rho_{f}=S_{w} \rho_{w}+\left(1-S_{w}\right) \rho_{h c},
$$


where, $\rho_{w}$ and $\rho_{h c}$ are the densities of water and hydrocarbon, respectively. Once $K^{*}, G^{*}$ and $\rho^{*}$ are known the velocities of $\mathrm{P}$ - and $\mathrm{S}$ - waves can be determined from the well-known formulas

$$
V_{P}=\sqrt{\frac{K^{*}+4 / 3 G^{*}}{\rho^{*}}},
$$

and

$$
V_{S}=\sqrt{\frac{G^{*}}{\rho^{*}}} \quad .
$$

\section{B.3.3. Sensitivity of $P$ - and $S$-wave velocities to porosity and saturation}

In the preceding section, we established the relationships between seismic velocities and bulk density with the rock's porosity and fluid saturation. In the following, we study the variation of seismic velocity field with fractional changes in porosity and saturation. In other words, we determine the sensitivities of both $\mathrm{P}$ - and $\mathrm{S}$-wave velocities to a variation of porosity and saturation. For sensitivity calculations, we make use of the following equations

$$
\begin{aligned}
& \frac{\partial V_{P}}{\partial \phi}=\frac{1}{2 \rho^{*} V_{P}}\left[\frac{\partial K^{*}}{\partial \phi}+\frac{4}{3} \frac{\partial G^{*}}{\partial \phi}\right]-\frac{V_{P}}{2 \rho^{*}} \frac{\partial \rho^{*}}{\partial \phi}, \\
& \frac{\partial V_{s}}{\partial \phi}=\frac{1}{2 \rho^{*} V_{s}} \frac{\partial G^{*}}{\partial \phi}-\frac{V_{s}}{2 \rho^{*}} \frac{\partial \rho^{*}}{\partial \phi}, \\
& \frac{\partial V_{P}}{\partial S_{w}}=\frac{1}{2 \rho^{*} V_{P}}\left[\frac{\partial K^{*}}{\partial S_{w}}+\frac{4}{3} \frac{\partial G^{*}}{\partial S_{w}}\right]-\frac{V_{P}}{2 \rho^{*}} \frac{\partial \rho^{*}}{\partial S_{w}} .
\end{aligned}
$$

and

$$
\frac{\partial V_{s}}{\partial S_{w}}=\frac{1}{2 \rho^{*} V_{s}} \frac{\partial G^{*}}{\partial S_{w}}-\frac{V_{s}}{2 \rho^{*}} \frac{\partial \rho^{*}}{\partial S_{w}}
$$


The necessary formulas calculating Fréchet derivative of $K^{*}, G^{*}$ and $\rho^{*}$ with respect to $\phi$ and $S_{w}$ are presented in Appendix-I. In the following we present a numerical experiment to study the effect of sensitivities on various lithological factors. We consider a model of sand with and without any clay content and saturated with either water or water and gas. In Figures 3.B.7 and 3.B.8 we present sensitivity curves with respect to porosity for fixed values of water saturation and clay content.
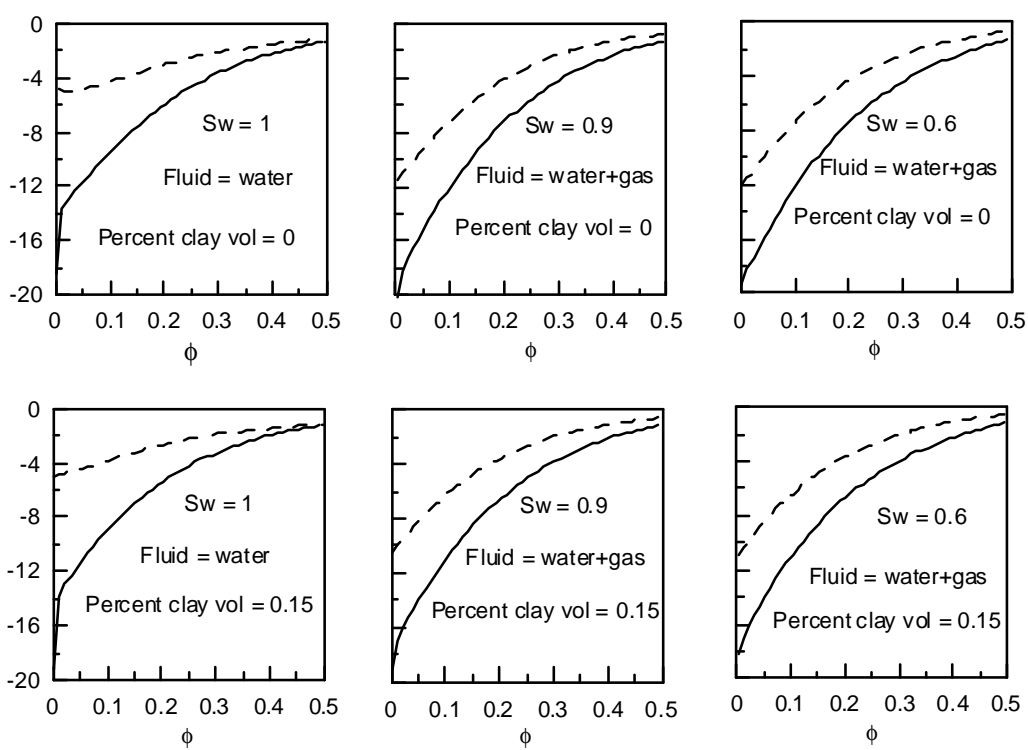

Figure 3.B.7. Plot of sensitivities for both P- (solid line) and S-wave (broken line) velocities with respect to porosity versus porosity for different values of saturation and clay content. Curves presented in the upper panel are for clean sand saturated with water or water and gas while curves in the lower panel are fordirty sand contaminated with $15 \%$ clay content Sensitivities for both P- and S-wave vary strongly with porosity for any value of saturation.

Note that in Figure 3.B.7 the sensitivities for both P- and S-wave velocities with respect to porosity vary strongly with porosity irrespective of the saturation or the lithological factor, such as clay content. Interestingly, a sharp variation of sensitivity of Svelocity with an increase in porosity is noticeable in both the upper and lower panels of the sensitivity plot. Such behavior is contrary to the general view that presence of gas affects little the S-velocity. On the other hand, in Figure 3.B.8 it is clear that for wide range of values of water saturation the variation of sensitivities both for P- and S-wave velocities 
remain constant with an increase in saturation except near the vicinity of high saturation values where there is an extremely sharp change in sensitivity values for both P- and S-wave velocities with the presence of gas.
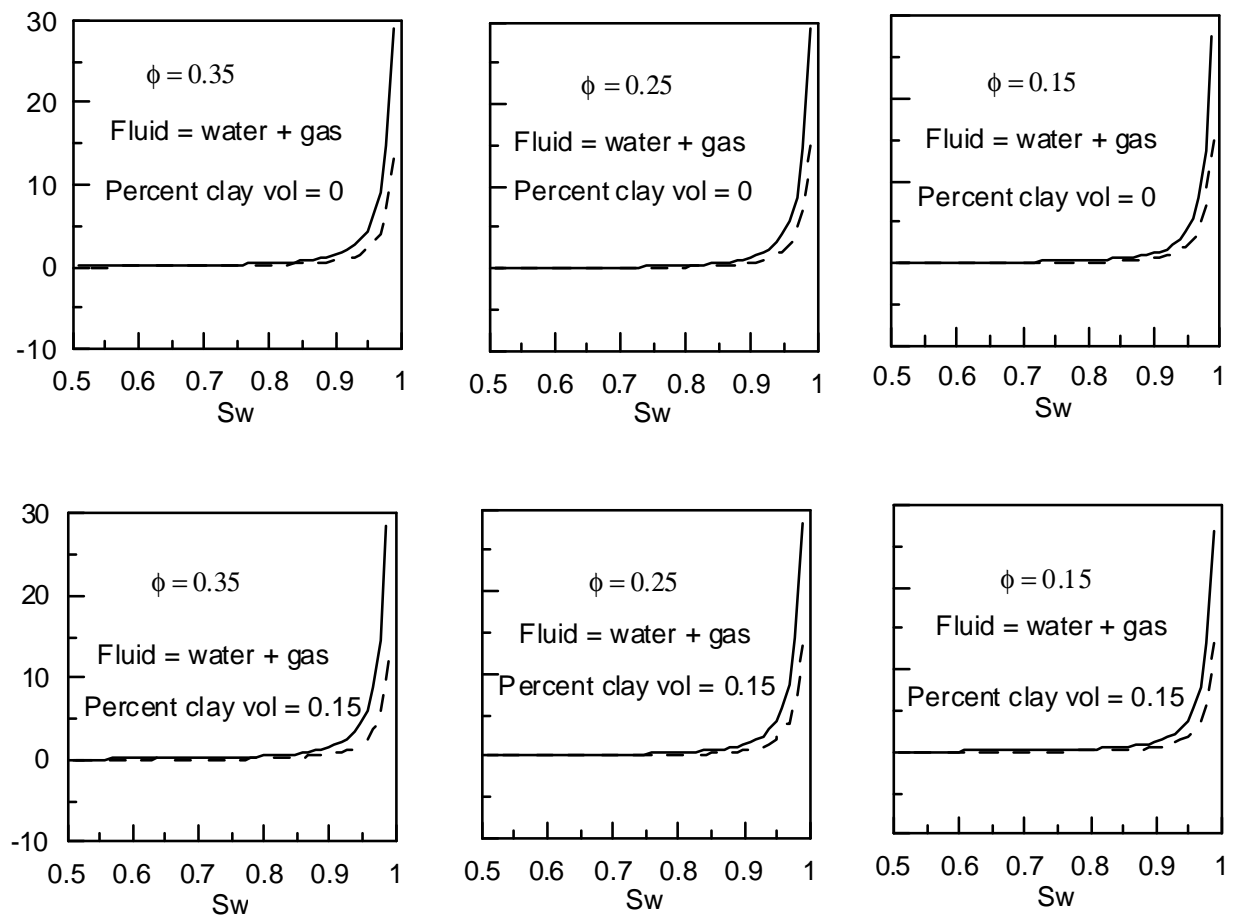

Figure 3.B.8. Plot of sensitivities for both P- (solid line) and S-wave (broken line) velocities with respect to saturation versus saturation for different value of porosity and clay content. Curves presented in the upper panel are for clean sand saturated with water or water and gas while curves in lower panel are for dirty sand contaminated with $15 \%$ clay. Both P- and Svelocity remain flat for most of the saturation value except rear to the higher end of saturation, where it shows a vary strong variation.

Figures 3.B.9(a) and 3.B.9(b) show plots of P- and S-wave velocities as functions of porosity for different values of saturation over a clean sand model. With a given saturation value, both P- and S-velocity curves decrease with an increase in porosity. Interestingly, presence of gas causes a higher rate of change for both $\mathrm{P}$ - and S-velocities. Note that further increase in gas volume has little effect on the change of velocity with porosity. In Figure 3.B.9(c) we 
(a)

(c)
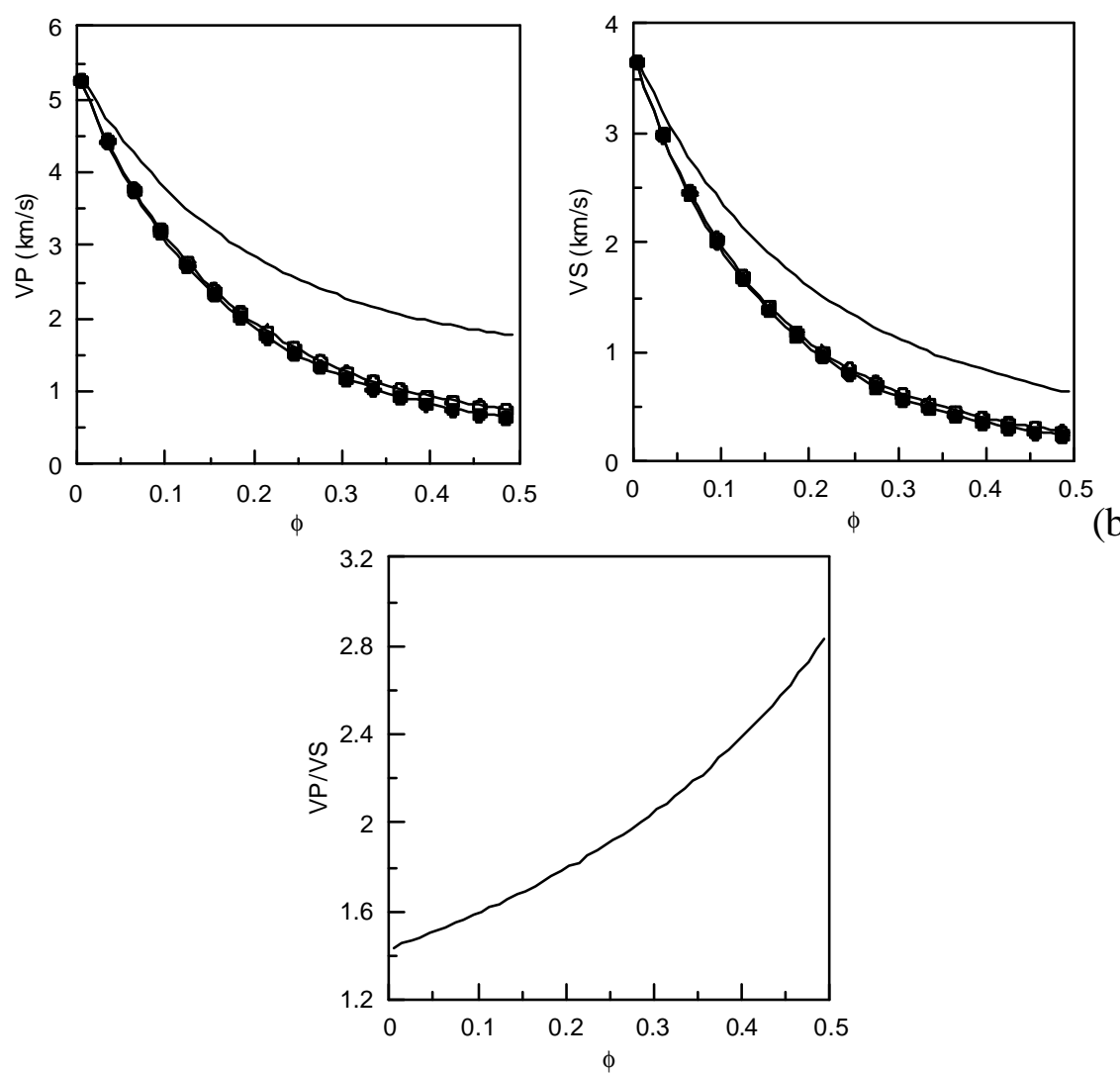

Figure 3.B.9. (a) Plot of $\mathrm{P}$ - velocities and (b) plot of $\mathrm{S}$ - velocities with respect to porosity for different values of saturation. The continuous line corresponds to $100 \%$ water saturation, the line with open circle corresponds to $90 \%$ saturation, and the line with filled circles corresponds to $60 \%$ saturation. Model considered is clean sand saturated either with water or water and gas. Panel (c) is the VP/VS ratio versus porosity, which continues to increase nonlinearly with porosity and the nature of curves remains the same irrespective of saturation.

plot $\mathrm{Vp} / \mathrm{Vs}$ ratio with respect to porosity. Note that $\mathrm{Vp} / \mathrm{Vs}$ ratio continues to increase non-

linearly with porosity irrespective of saturation. To develop a comprehensive idea about the

characteristics of both $\mathrm{P}$ - and S-wave velocities with porosity and saturation we present color

filled contour maps in Figures 3.B.10(a) and 3.B.10(b). 

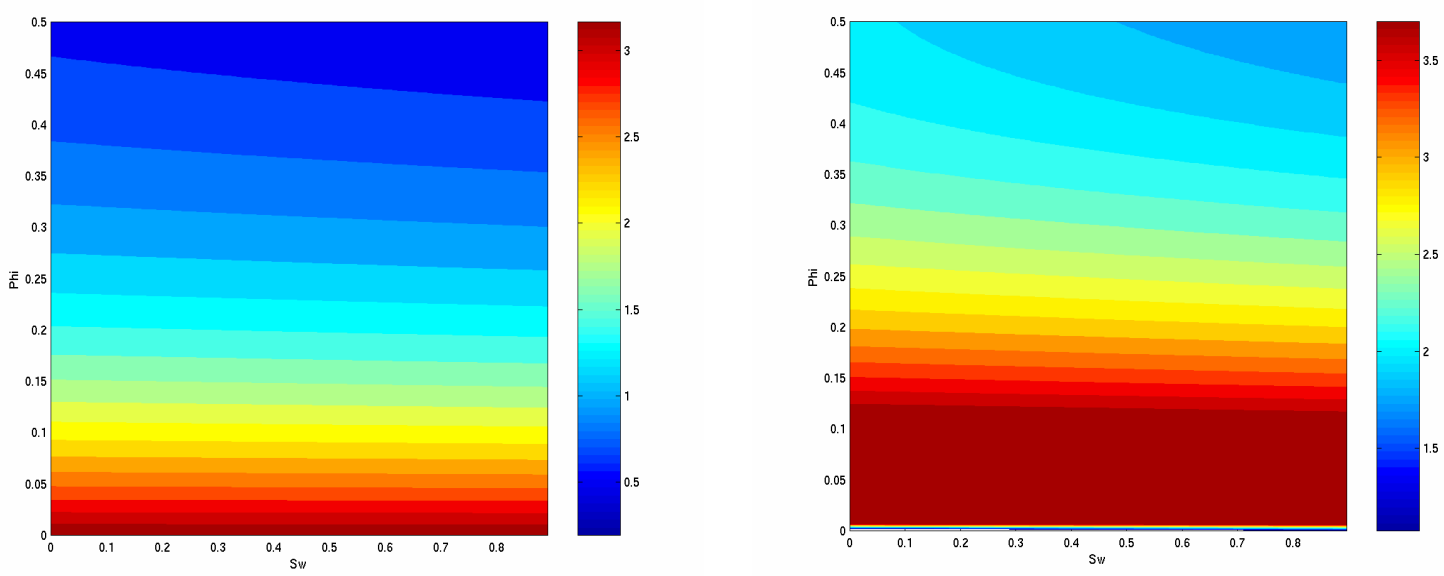

Figure 3.B.10. (a) Contour maps of $\mathrm{P}$ - velocity and (b) contour maps of $\mathrm{S}$ - velocity as functions of porosity and saturation.

The above contours maps show either low slope or else remain constant for a wide range of saturation values. This immediately suggests that the resolving limit of saturation from waveform inversion will also be very small.

\section{B.3.4. Determination of petrophysical parameters and seismic velocities from well logs}

In our study, we consider a set of well-log measurements acquired in the deepwater Gulf of Mexico. We make use of nuclear logs (both neutron and bulk density) and acoustic logs to determine the effective porosity of the rock formations while we use deep resistivity and porosity logs to determine water saturation using the Simandoux model. We also use the natural gamma log to estimate the percent volume of shale. In Figure 3.B.11, we present wireline- log derived porosity, saturation and percent clay content of the geological section. The log indicates presence of shale, sand sequence, and possibility of having shaly-sand or sandy shale formations. In our analysis, we consider the following log as the reference for true effective porosity and saturation of the area. In Figure 3.B.12, we present P-, Svelocities and density obtained from sonic and density logs and the corresponding values 
calculated using petrophysical formulas as discussed in the preceding section. Note that the predicted values of P-, S-velocities, and bulk density are in good agreement with the logmeasured values.
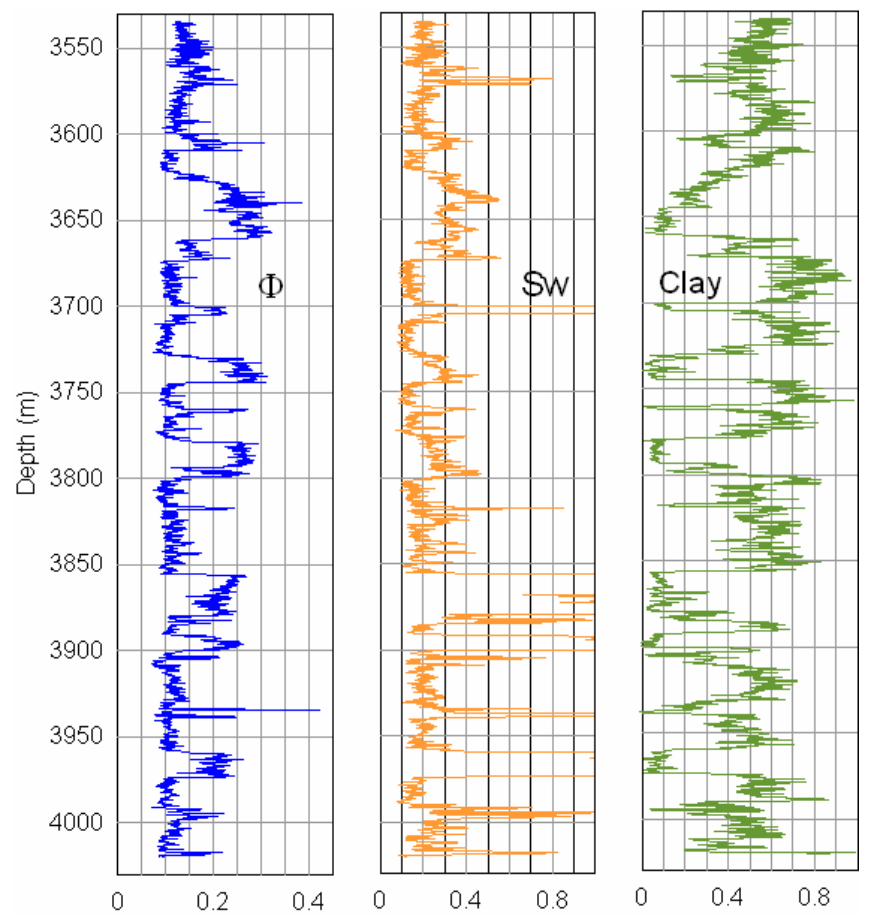

Figure 3.B.11. Plots of wireline-log-derived porosity (blue line), water saturation (orange line) and percent clay volume (green line) with depth 


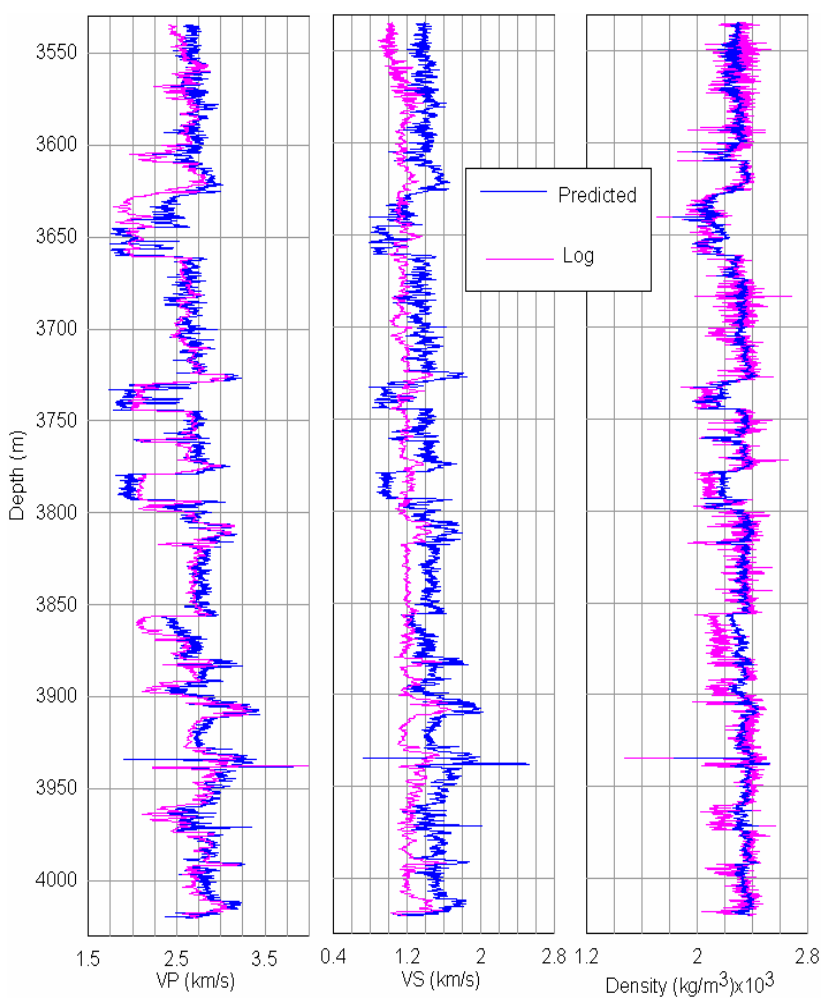

Figure 3.B.12. Plots of log-measured (magenta line) and predicted (blue line) values of P-, Svelocities and bulk density with depth.

In our numerical experiment, we consider those predicted values of velocities and density to be the true representations of the subsurface. We then convert these logs (Figure 3.B.12) from depth to seismic time the computation of the synthetic seismic wave field. Time converted profiles of P-, S-velocities and density are shown in Figure 3.B.13. 


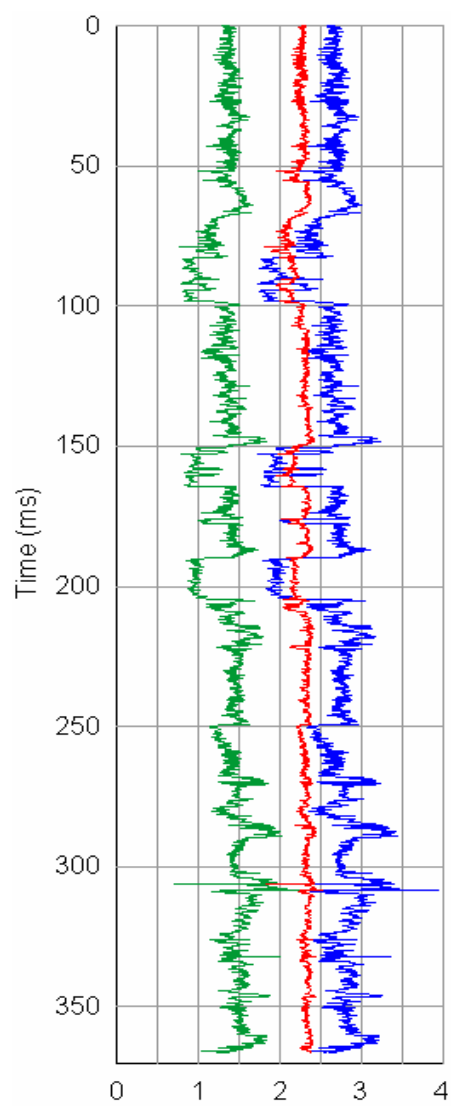

Figure 3.B.13. Plot of predicted values of P-, S-velocities and bulk density with two-way vertical travel time in $(\mathrm{ms})$.

\section{B.3.5. Waveform inversion in the determination of petrophysical parameters}

At the outset of our numerical experiment with waveform inversion, we generate seismic gathers in the $\tau-p$ domain using the velocity and density profiles shown in Figure 3.B.13. To obtain a realistic $\tau-p$ seismic section we embed the above profiles by adding water and thick shale layers at the top, mimicking a marine geometry. We then generate a synthetic $\tau$ - $p$ seismic section with a 4 ms sampling interval using a $40 \mathrm{~Hz}$ Ricker wavelet. The synthe tically generated $\tau-p$ seismic section is shown in Figure 3.B.14. 


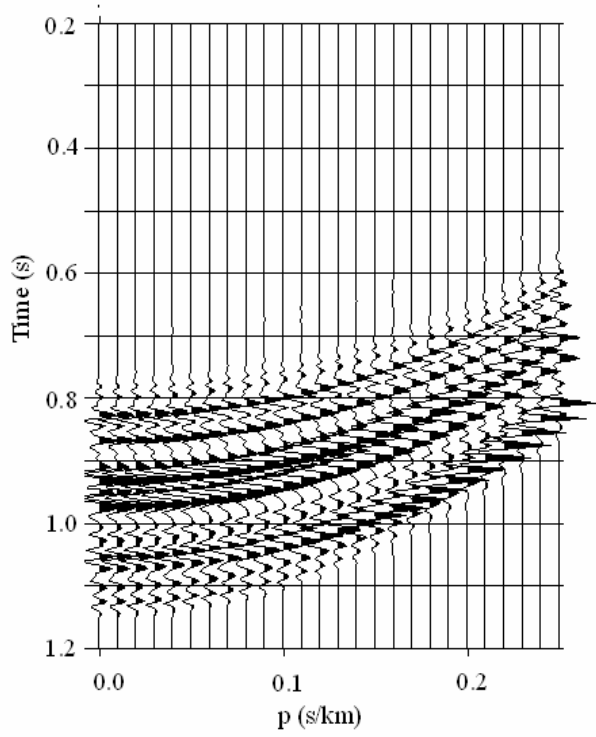

Figure 3.B.14. Synthetically generated $\tau-p$ seismic section using the time converted velocities and the density profile shown in Figure 3.B.7.

It is well recognized that, because the seismic signal is band limited, the vertical resolution of the seismic derived model is limited within the seismic scale. However, we consider a fullscale over-parameterized model in which each layer has a thickness equal to $4 \mathrm{~ms}$ two-way time. We then invert the $\tau-p$ seismic section using an adaptively regularized, truncated Gauss-Newton inversion algorithm (Sen and Roy, 2003; Roy et al., 2003) assuming a constant half-space value of porosity and saturation as the a priori petrophysical model. For comparison with the true value of porosity and saturation, we first make a depth to time conversion of porosity and saturation logs and then resample them to $4 \mathrm{~ms}$ as a coarse representation. We present our inverted results as logs with $4 \mathrm{~ms}$ sampling interval in Figure 3.B.15. Note that the estimated porosity is in good agreement with the true value. However, 
the algorithm fails to retrieve the true saturation values. This is expected based on our study of sensitivities reported in the previous section. Figure 3.B.16 indicates a very good data- fit.
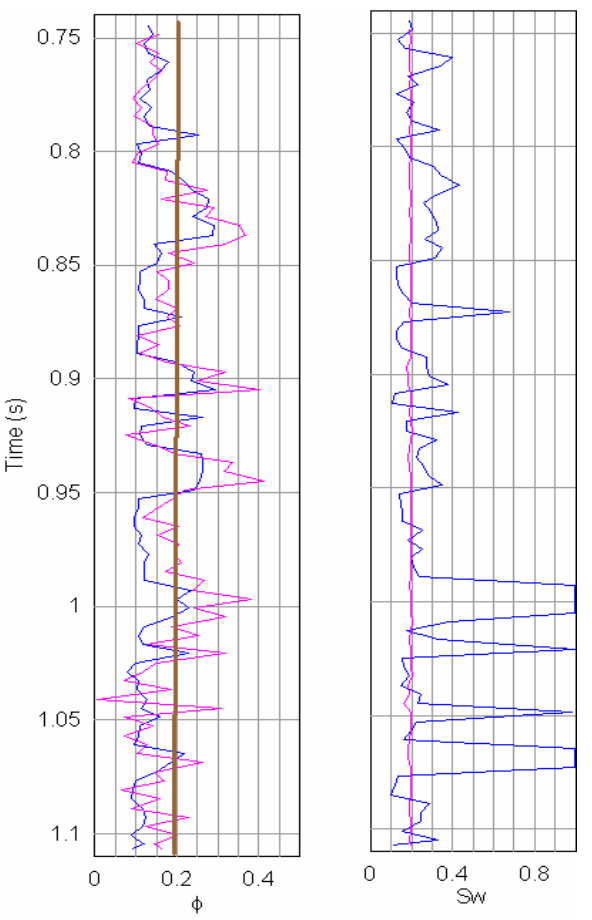

Figure 3.B.15. Plot of inverted porosity profile (magenta line) overlaid on true profile (blue line).

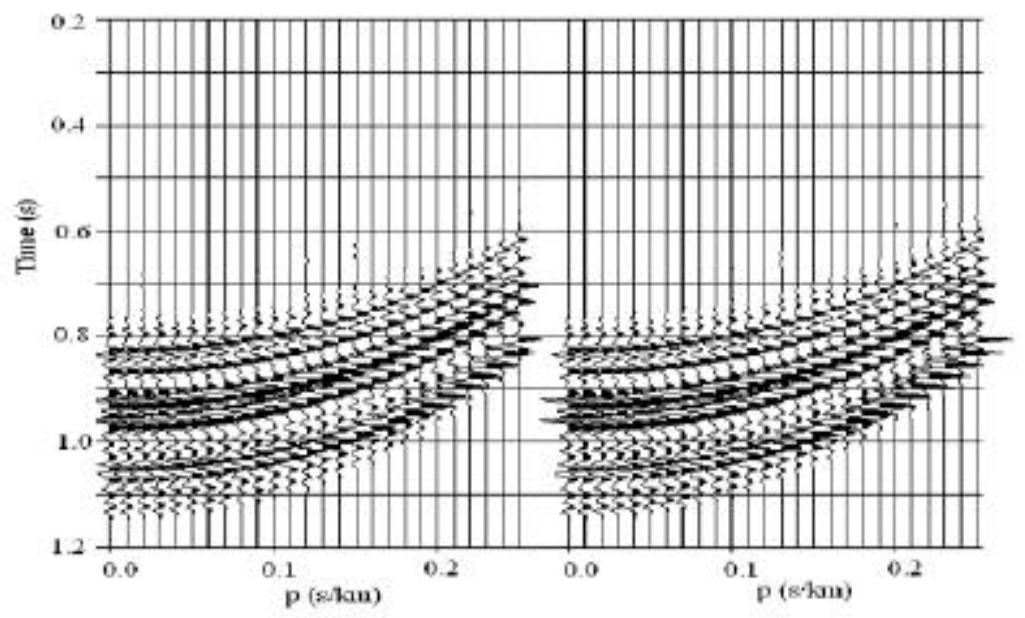

Figure 3.B.16. Plot of $\tau-p$ seismic section after inversion (left panel) and the original one (right panel) 


\section{B.3.6. Conclusions}

We studied the feasibility of using full waveform inversion of pre-stack seismic data in the direct estimation of petrophysical parameters such as porosity and saturation. In determining the relationship between elastic moduli with porosity and saturation we use Lee's (2002) extension of the Biot-Gassmann model. With the sensitivity study we observe that the sensitivity of porosity to the seismic velocities (both $\mathrm{P}$ - and $\mathrm{S}$ ) is considerable while that of the saturation is insignificant. This reflects in our inversion results. While it is possible to make a robust estimate of the porosity even with a flat prior, the algorithm fails to make any appropriate estimate of the saturation despite an excellent data fit.

\section{B.3.7. Appendix I}

Using equations (3.B.15) and (3.B.16), we can write

$$
K^{*}=K_{m}(1-\beta)+K_{m} \frac{F\left(S_{w}\right) \beta}{K_{m} \phi+F\left(S_{w}\right)(\beta-\phi)},
$$

where

$$
F\left(S_{w}\right)=\frac{K_{w} K_{h c}}{\left(K_{w}-K_{h c}\right) S_{w}+K_{w}}=\frac{N}{D} .
$$

Taking partial derivatives with respect to $\phi$ and $S_{w}$ in (3.B.27) and using (3.B.28) we obtain

$$
\frac{\partial K^{*}}{\partial \phi}=-K_{m} \frac{\partial \beta}{\partial \phi}+K_{m} \frac{F\left(S_{w}\right) \frac{\partial \beta}{\partial \phi} D-\left[K_{m}+F\left(S_{w}\right)\left(\frac{\partial \beta}{\partial \phi}-1\right)\right] N}{D^{2}},
$$

and

$$
\frac{\partial K^{*}}{\partial S_{w}}=K_{m} \frac{F^{\prime}\left(S_{w}\right)[\beta(D-N)+\phi N]}{D^{2}},
$$

where, by taking the partial derivative with respect to $\phi$ in equation (3.B.13), 


$$
\frac{\partial \beta}{\partial \varphi}=-\frac{\left(A_{1}-A_{2}\right) \exp \left[\left(\phi+\tau_{1}\right) / \tau_{2}\right]}{\tau_{2}\left\{1+\exp \left[\left(\phi+\tau_{1}\right) / \tau_{2}\right]\right\}^{2}}
$$

and by taking the derivative with respect to $S_{w}$ in equation (3.B.28),

$$
F^{\prime}\left(S_{w}\right)=-\frac{\left(K_{h c}-K_{w}\right) K_{w} K_{h c}}{\left[\left(K_{h c}-K_{w}\right) S_{w}+K_{w}\right]^{2}}
$$

Again in equation (3.B.17) for the sake of simplicity we express $G^{*}$ as

$$
G^{*}=\frac{G_{n}^{*}}{G_{d}^{*}},
$$

where,

$$
G_{n}^{*}=G_{m} K_{m}(1-\beta)(1-\phi)^{2}+G_{m} \beta^{2} \xi(1-\phi)^{2},
$$

and

$$
G_{d}^{*}=K_{m}+\frac{4}{3} G_{m}\left[1-(1-\phi)^{2}\right]
$$

Now, from (3.B.7)

$$
\partial_{x}\left[G^{*}\right]=\frac{G_{d}^{*} \partial_{x}\left[G_{n}^{*}\right]-G_{n}^{*} \partial_{x}\left[G_{d}^{*}\right]}{\left[G_{d}^{*}\right]^{2}},
$$

where the derivative index, $x$, can be either $\phi$ or $S_{w}$. We may then write

$$
\begin{aligned}
\partial_{\phi}\left[G_{n}^{*}\right]= & -2 G_{m} K_{m}(1-\beta)(1-\phi)-G_{m} K_{m}(1-\phi)^{2} \frac{\partial \beta}{\partial \phi} \\
& -2 G_{m} \beta^{2 \xi}(1-\phi)+2 G_{m} \beta \xi(1-\phi)^{2} \frac{\partial \beta}{\partial \phi} \\
& +G_{m} \beta^{2}(1-\phi)^{2} \frac{\partial \xi}{\partial \phi}
\end{aligned}
$$


where

$$
\frac{\partial \xi}{\partial \phi}=-\frac{K_{f} K_{m}\left[K_{f}\left(\frac{\partial \beta}{\partial \phi}-1\right)+K_{m}\right]}{\left[K_{f}(\beta-\phi)+K_{m} \phi\right]^{2}},
$$

and

$$
\partial_{\phi}\left[G_{d}^{*}\right]=-\frac{8}{3} G_{m}(1-\phi)
$$

Similarly,

$$
\partial_{S w}\left[G_{n}^{*}\right]=G_{m} \beta^{2}(1-\phi)^{2} \frac{\partial \xi}{\partial S_{w}},
$$

where

$$
\frac{\partial \xi}{\partial S_{w}}=-\frac{F^{\prime}\left(S_{w}\right)\left[K_{m}-(\beta-\phi) K_{f}\right]}{\left[K_{f}(\beta-\phi)+K_{m} \phi\right]^{2}},
$$

and

$$
F^{\prime}\left(S_{w}\right)=-\frac{K_{w} K_{h c}\left(K_{h c}-K_{w}\right)}{\left[\left(K_{h c}-K_{w}\right) S_{w}+K_{w}\right]^{2}}
$$

Again, from 3.B.18 and 3.B.19 we get

$$
\frac{\partial \rho^{*}}{\partial \phi}=\rho_{f}-\rho_{m},
$$

and

$$
\frac{\partial \rho^{*}}{\partial S_{w}}=\phi\left(\rho_{w}-\rho_{h c}\right)
$$




\section{CONCLUSIONS}

During the third year of this project, we built upon the development carried out in the previous two years to develop methodologies for the integration of 3D seismic data, pre-stack and post-stack, with time records of fluid production measurements. Efficient implementation of joint inversion of seismic and fluid flow data depend on the efficient forward modeling of seismic and fluid flow, realistic mapping of petrophysical parameters to elastic parameters, and efficient algorithms for inversion. All these aspects were tested rigorously during this year. We implemented a full waveform inversion algorithm (that includes all internal multiples and converted waves) in the $\tau$-p domain on a cluster of personal computers. We found that our algorithm is highly scalable and shows almost a linear speedup up to 16 nodes on a cluster. Thus we were able to develop a very efficient and accurate algorithm for full waveform inversion that can be used routinely for seismic data analysis. Most seismic inversion algorithms provide with maps of elastic properties which are then mapped to petrophysical parameters using simple empirical formulas. To address this we developed a rigorous waveform inversion algorithm that directly provides flow parameters. We employed a modified Biot-Gassmann equation that was validated with a suite of well logs from the Gulf of Mexico. Analytic formulae were developed for computing sensitivity of seismograms to porosity and saturation. We found that the porosity is very well determined but the seismic data are insensitive to the changes in saturation. Finally we developed a novel, efficient algorithm to estimate spatial distributions of porosity and permeability by jointly inverting $3 \mathrm{D}$ pre-stack seismic data and time records of fluid production measurements. This algorithm was successfully tested on synthetic data contaminated with random noise. It was also found that fluid production measurements acquired in 
production wells often don't have the degrees of freedom necessary to accurately estimate permeability distributions in the inter-well region. However, the use of pre-stack seismic data helps improve the resolution of porosity maps in the inter-well regions. 


\section{REFERENCES}

Abacuioglu, Y. D., Oliver, D. S., and Reynolds, A. C. Efficient reservoir matching using subspace vectors: Computational Geosciences, 5, 151-172, 2001.

Amdahl, G. Keynote address, Second International Supercomputer Conference, Santa Clara, CA, May 1987.

Behrens, R. A., Tran, T. T. Incorporating seismic data of intermediate vertical resolution into 3D reservoir: SPE Annual Technical Conference and Exhibition, New Orleans, Louisiana, 27-30 September, 1998.

Biot, M. A. General theory of three dimensional consolidation: Jl. Appl. Physics, 12:155-164, 1941.

Biot, M. A. Generalized theory of acoustic propagation in porous dissipative media: J. Acoust. Soc. Am., 34:1254-1264, 1962.

Biot, M. A. Theory and propagation of elastic waves in a fluid saturated porous solid. I. Low frequency range: J. Acoust. Soc. Am., 28:168-178, 1956 .

Biot, M. A. Theory of propagation of elastic waves in fluid saturated porous solid, II Higher frequency range: J. Acoust. Soc. Am., 28:179-191, 1956 .

Chu, L., Reynolds, A. C., and Oliver, D. S. Reservoir description from static and well-test data using efficient gradient methods: SPE 29999, International Meeting on Petroleum Engineering, Beijing, PR China, 14-17, November, 1995.

Dembo R.S., Steihaug T. Truncated-Newton algorithms for large scale unconstrained optimization. Mathematical Programming, 26:190-212, 1983.

Gassmann, F. Uber die elastizitat poroser medien: Verteljahrsscrift der Naturforschenden Gesellschaft in Zurich, 96:1-23, 1951.

Grijalba-Cuenca, A., and Torres-Verdin, C. Geostatistical inversion of 3D seismic data to extrapolate wireline petrophysical variables laterally away from the well: SPE Annual Technical Conference and Exhibition. Dallas, Texas, 1-4 October, 2000.

Hamilton, E. L. Elastic properties of marine sediments: Jl. Geophys. Res., 76, 579-604, 1971.

Hass, A., and Dubrule, O. Geostatistical inversion -a sequential method for stochastic reservoir modeling constrained by seismic data: First Break, 12:561-569, 1994.

Hill, R. The elastic behavior of crystalline aggregate: Proc. Phys. Soc. London Ser. A, 65:349-354, 1952.

Kennett, P. R., Sambridge, M. S., and Williamson, P. R. Subspace methods for large inverse problems with multiple parameter classes: Geophys. J. Int., 94:237-247, 1988.

Kolb, P., Collino, F., and :Lailly, P. Pre-stack inversion of a 1-D medium: proceedings of the IEEE, 74:498-508, 1986. 
Lee, M.W. Biot-Gassmann theory for velocities of gas hydrate bearing sediments: Geophysics, 67, 1711-1719, 2002.

Mora, P. Elastic wave-field inversion of reflection and transmission data: Geophysics, 53:750-759, 1988.

Nash S.G. A survey of truncated Newton methods. Journal of Computational and Applied Mathematics 2000; 124:45-59.

Nur, A.M., and Wang, Z. Seismic and acoustic velocities in reservoir rock Volume.1, experimental studies: Geophysics Reprint Series, Franklyn K. Levin, Tulsa, Oklahoma, Society of Exploration Geophysicists, 1998.

Oldenburg, D. W., McGillivrary, P. R., and Ellis, R. G. Generalized subspace methods for la rgescale inverse problems: Geophys. J. Int., 114, 12-20, 1993.

Oliver, D. S. Incorporation of transient pressure data into reservoir characterization: In Situ, 18, 234275, 1994.

Reuss, A. Berechnung der fliessgrense von mischkristallen auf grund der plasuzitabedinggung fur einkristalle: Zeitschrift fur Angewandte Mathematik aus Mechanik, 9, 49-58, 1929.

Roy I. G., Sen M. K., Torres-Verdin C., Varela O. J. Pre-stack inversion of Gulf of Thailand data. Geophysics 2003 (submitted).

Roy I. G.. A robust descent type algorithm for geophysical inversion through adaptive regularization. Applied Mathematical Modelling 2002; 26:610-634.

Roy, I. G., Sen, M. K., Torres-Verdin, C., and Varela, O. J. Pre-stack inversion of Gulf of Thailand data, Geophysics, 2003 (under review).

Sen M. K., Roy I. G. Computation of differential seismograms and iteration adaptive regularization in pre-stack waveform inversion. Geophysics, 6:2026-2039, 2003.

Sen V, Sen MK, Stoffa PL. PVM based 3-D Kirchhoff depth migration using dynamically computed travel times: An application in seismic data processing. Parallel Computing, 1999; 25:231248.

Sen, M. K. and Roy, I. G. Computation of differential seismograms and iteration adaptive regularization in pre-stack waveform inversion, Geophysics, 2003 (in press).

Sen, M. K., and Stoffa, P. L. Nonlinear one-dimensional seismic waveform inversion using simulated annealing: Geophysics, 56:1624-1638, 1991.

Snir M., Otto S., Huss-Lederman S., Walker D., and Dongarra J. MPI: The Complete Reference, MIT Press, Cambridge, MA, 1996.

Tarantola, A. Inverse Problem Theory-Methods for Data Fitting and Model Parameter Estimation: Elsevier Publishing Company, 1987.

Tikhonov, A. N. and Arsenin, V. A. Solutions of Ill-posed Problems, Winston \& Sons, Washington, 1977. 
Voigt, W. Lehrbuch der Kristallphysik: Teubner, 1928.

Wang, Z. The Gassmann's equation revisited: Compairing laboratory data with Gassmann's predictions, in Wang, Z. and Nur, A., Eds, Seismic and acoustic velocities in reservoir rocks, 3: Recent developments: Soc. Expl. Geophys., 8-23, 2000.

Wood, A. B. A text book of sound: G. Bell and Sons, 1941.

Wu, Z., Alpak, F. O., and Torres-Verdín C. A quantitative study to assess the value of pressure data acquired with in-situ permanent sensors in complex $3 \mathrm{D}$ reservoir models subject to twophase fluid flow, SPE 84375, Annual Technical Conference and Exhibition. Denver, Colorado, U.S.A., 5-8 October 2003.

Wu, Z., Reynolds, A. C. and Oliver D. S. Conditioning geostatistical models to two-phase production data: SPE Journal, 4:142-154, 1999.

$\mathrm{Xu}, \mathrm{S}$. and White, R. E. A new velocity model for clay-sand mixtures, Geophysical Prospecting, 43:91-118, 1995.

Zhang, F. Z., Reynolds, A. C., and Oliver, D. S. Model errors inherent to conditioning a stochastic channel to pressure data: SPE 62987, Annual Technical Conference and Exhibition. Dallas, Texas, 1-4 October 2000. 


\section{APPENDICES}

\section{A. Manuscripts Submitted for Publication in R EFEREed TeChNiCal Journals}

Varela, O. J., Torres-Verdín, C., Sen, M. K., and Roy, I. G., 2003, Using time-lapse 3D seismic data to detect dynamic reservoir behavior due to water-oil displacement: a numerical study based on inversion, submitted for publication, Geophysics.

Varela, O. J., Torres-Verdín, C., and Sen, M. K., 2003, Enforcing smoothness and assessing uncertainty in nonlinear one-dimensional pre-stack seismic inversion: $\underline{\text { submitted for }}$ publication, Geophysical Prospecting

Varela, O. J., Torres-Verdín, C., and Sen, M. K., 2003, Joint stochastic inversion of pre-stack seismic data and well logs for high-resolution reservoir delineation: submitted for publication, Geophysics

Roy. I. G., Sen, M. K., and Torres-Verdín, C., 2004, Se ismic waveform inversion on a cluster of personal computers, Concurrency and Computation, in press.

\section{B. EXPANDED R EFERENCED CONFERENCE ABSTRACTS}

Varela, O. J., Torres-Verdín, C., and Sen, M. K., 2003, Joint stochastic inversion of pre-stack seismic data and well logs for high-resolution reservoir delineation and improved production forecast (Expanded Abstract): Society of Exploration Geophysicists (SEG) 73th Ann. Internat. Mtg., Dallas, Texas, October 26-31.

Varela, O. J., Torres-Verdín, and Sen, M. K., 2003, Enforcing smoothness and assessing uncertainty in one-dimensional pre-stack seismic inversion (Expanded Abstract): European Association of Geoscientists and Engineers (EAGE) 65th Ann. Conference and Exhibition, Stavanger, Norway, June 2-6. 